\title{
A DIGITAL MICROFLUIDIC APPROACH TO PROTEOMIC SAMPLE PROCESSING
}

by

VIVIENNE NANCY LUK

A thesis submitted in conformity with the requirements

for the degree of Doctor of Philosophy

Department of Chemistry

University of Toronto

(C) Copyright by Vivienne Nancy Luk (2012) 


\section{ABSTRACT}

\section{A Digital Microfluidic Approach to Proteomic Sample Processing}

Vivienne N Luk

Doctor of Philosophy

Department of Chemistry

University of Toronto

2012

Proteome profiling is the identification and quantitation of all proteins in biological samples. An important application of proteome profiling that has received much attention is clinical proteomics, a field that promises the discovery of biomarkers that will be useful for early diagnosis and prognosis of diseases. While clinical proteomic methods vary widely, a common characteristic is the need for (i) extraction of proteins from complex biological fluids and (ii) extensive biochemical processing (reduction, alkylation and enzymatic digestion) prior to analysis. However, the lack of standardized sample handling and processing in proteomics is a major limitation for the field. The conventional macroscale manual sample handling requires multiple containers and transfers, which often leads to sample loss and contamination. For clinical proteomics to be adopted as a gold standard for clinical measures, the issue of irreproducibility needs to be addressed. A potential solution to this problem is to form integrated systems for sample handling and processing, and in this dissertation, I describe my work towards realizing this goal using digital microfluidics (DMF). DMF is a technique characterized by the manipulation of discrete droplets $(100 \mathrm{~nL}-10 \mu \mathrm{L})$ on an array of electrodes by the application of electrical fields. It is well-suited for carrying out rapid, sequential, miniaturized automated biochemical assays. This thesis demonstrates how DMF can be a powerful tool capable of automating several protein handling and processing steps used in proteomics. 
Methods for implementing proteomic multi-step solution-phase processes (reduction, alkylation, and digestion) on DMF were developed. This was accompanied by the development of heterogenous enzymatic microreactors for efficient protein digestion. Strategies for reducing non-specific adsorption were developed. Finally, in proof-of-concept work, a combination of protein extraction and protein processing was demonstrated on DMF devices. 


\section{ACKNOWLEDGEMENTS}

I would first like to express my deepest gratitude to my supervisor, Professor Aaron Wheeler, for initiating such an exciting project. This work would not be possible without his guidance, continual support, enthusiasm, wisdom, and patience. More importantly, I would not be the scientist I am today without his teachings and philosophy on science.

I am also deeply grateful to my supervisory committee members, Professor Krull and Professor Jockusch. Their insights and suggestions were very much appreciated. The committee meetings were a rewarding experience and I always walked away with new perspectives on science and additional tools for research. Likewise, I would like to acknowledge Professor Eugenia Kumacheva for participating in my comprehensive oral examination.

I would like to thank the entire Wheeler lab for their friendship, supportive environment, and scientific exchange of knowledge. I would not have survived graduate school without their support. I especially thank Andrea, Irena, Lindsey, Mark, Mohamed, Ryan, Sam, and Steve. To Andrea - thank you for all your baked goods, you made my life that much more sweeter; to Irena - you are an insirpation to all women who want to be a successful professional and a caring mother; to Lindsey - my motivator and competitor to all things fit and healthy; to Mark - for simply being Mark; to Mohamed - thank you for being my microfabrication advisor; to Ryan (a.k.a. Mr. Chill) - thank you for always cutting the tension in the lab; to Sam - thank you for being a great desk-mate and for creating tension in the lab; and to Steve - thank for hosting awesome lab parties. I would also like to thank Beth, Edmond, Mais, Mike, and Sergio, who were part of the Wheeler lab from the very beginning.

Last by not least, I am indebted to my family for their constant support and patience throughout my graduate carrer. To my parents, Leo and Petty Luk, I thank them for always putting me first. To my sister, Victoria Luk, I thank her for putting up with me and with my ridiculous demands during her time as my undergraduate researcher assistant. And finally to my partner, Ronald Soong, I thank him for putting me back on track, always assisting me in times of struggle and despair. 


\section{OVERVIEW OF CHAPTERS}

This thesis describes the development and adaptation of proteomic sample preparation methodologies on digital microfluidic systems. The thesis describes the various obstacles and approaches taken to overcome and enhance the development of digital microfluidics-based sample handling and processing of proteomic samples while in the Wheeler Group. Here, for the benefit of the reader, I have provided a brief outline of each chapter's contents.

Chapter one provides a literature review of microfluidics-based methods for the handling and processing of proteomic samples to enable highly reliable, comprehensive identification of proteins. First, I present a brief overview of proteome profiling, with a short review of common methodologies currently used in laboratories. Second, I introduce microfluidic technologies as a useful tool for proteomic sample preparation. Third, I review several microfluidics platforms that have been developed for proteomic sampling handling and processing.

Chapter two describes a practical and straightforward method for limiting non-specific adsorption on digital microfluidic devices. This method uses a low concentration of a pluronic additive in solution. Two orthogonal techniques, confocal microscopy and ToF-SIMS, were used to evaluate the utility of pluronics as an additive in preventing non-specific adsorption. This strategy allows for the actuation of droplets containing greater than 1000-fold higher protein concentrations than is possible without the additive.

Chapter three describes a microfluidic system integrating several common processing steps in proteomic analysis, including reduction, alkylation, and enzymatic digestion. MALDI-MS and fluorogenic assays were used to qualitatively evaluate DMF-driven proteomic reactions and quantitatively evaluate reproducibility of each reaction step, respectively. This work demonstrated that digital microfluidics is a powerful tool for rapid, reproducible, automated sample processing of proteomic samples.

Chapter four demonstrates the capacity of the combination of DMF and hydrogels for forming enzyme-embedded microreactors. Agarose discs were formed and were modified to bear covalently attached trypsin. Fluorogenic assays were used to optimize the covalent modification procedure for maximum enzymatic digestion efficiency, with the highest efficiency observed at $31 \mu \mathrm{g}$ trypsin per $2 \mathrm{~mm}$-diameter agarose gel disc. Gel discs prepared in this manner were used 
in an integrated method in which proteomic samples were sequentially reduced, alkylated, and digested, with all sample and reagent handling controlled by digital microfluidic droplet operation. This work represents the first implementation of hydrogel-based enzyme microreactors for DMF and a useful new tool for miniaturized, automated proteomic sample processing.

Chapter five represents work in progress, and it describes a methodology for DMF-based proteomic sample processing, integrating six proteomic processing steps, including protein extraction, clean-up, resolubilization, reduction, alkylation, and digestion. In this method, a model serum sample containing HSA, IgG, transferrin, IgA, and haptoglobin (all at their physiological concentrations) was used to evaluate and compare the reproducibility of DMFdriven method to macroscale (pipetting) method. In initial work, the reproducibility of the method was evaluated using spectral counting, with the goal of eventual optimization according to the guidelines set out by the Human Proteome Organization (HuPO) for proteomic sample preparation.

The Appendix describes an extra project that is outside of the overall narrative of this thesis. It introduces the marriage of DMF with hydrogel-based enzyme reactors and cell culture scaffolds that allow for fast and efficient multi-step processes. In this work, $\sim 2 \mathrm{~mm}$ diameter hydrogel discs were formed and used in DMF devices. Several fundamental properties of gel discs in DMF systems were evaluated, including adhesion to the device surfaces, the nature of actuation of droplets to and from gel discs, and the transport of material through gel discs. This system was demonstrated to be useful for generating integrated enzymatic microreactors and for threedimensional cell culture. 


\section{OvERVIEW OF AUTHOR CONTRIBUTions}

During the course of my research, I was fortunate to work with a number of collaborators in and out of the Wheeler group, many of whom are co-authors on the journal papers that have been published or are in press. Here I outline the contributions each person made towards the work presented herein.

In the work described in Chapter two , Dr. Gary Mo (then a graduate student of Prof. Chris Yip) assisted in the operation of the confocal microscope and acquisition of confocal-related data. Dr. Ronald Soong (then a graduate student of Prof. Peter Macdonald) assisted in the collection and interpretation of diffusion data via NMR spectroscopy. I proposed (and implemented) the idea of using pluronics as a solution additive to prevent non-specific adsorption on DMF devices. I fabricated DMF devices, characterized non-specific adsorption using ToF-SIMS and confocal microscopy, probe the limits of pluronics as an additive at preventing non-specific adsorption, and analyzed all of the confocal and mass spectrometry data. This work was published in Langmuir (Luk, V.N., Mo, G.C. \& Wheeler, A.R. Pluronic additives: a solution to sticky problems in digital microfluidics. Langmuir 24, 6382-6389 (2008)).

The experimental work and planning of $\underline{\text { Chapter three }}$ was primarily carried out me. This work was published in Analytical Chemistry (Luk, V.N. and Wheeler, A.R. A digital microfluidic approach to proteomic sample processing. Analytical Chemistry 81, 4524-30 (2009)).

In the experiments described in Chapter four, I worked with Dr. Fiddes (then a graduate student of Prof. Eugenia Kumacheva and Prof. Aaron Wheeler) who assisted in the development of hydrogel discs as a solid support for the immobilization of enzymes. Dr. Fiddes participated in helpful discussions and supported the initial exploration of various hydrogel formats for the immobilization of enzymes on hydrogel supports and the collection of confocal data. Victoria Luk (then an undergraduate summer student) helped with the fabrication of enzymatic hydrogel microreactors and together we carried out the digestion assays. I proposed (and implemented) the idea of using hydrogels as a solid support for heterogenous enzymatic digestion, developed a protocol for the immobilization of enzymes on hydrogel discs, fabricated DMF devices, and collected and analyzed all of the fluorogenic assay data and mass spectrometry data. Dr. Fiddes and I are are co-first-authors on a paper describing this work in Proteomics (Luk, V.N., Fiddes, 
L.F., Luk, V.M., Kumacheva, E., Wheeler, A.R. Digital Microfluidic-Hydrogel Microreactors for Proteomics. Proteomics in press 2012 DOI:10.1002/pmic.201100608).

Chapter five describes initial work that I carried out, working towards a fully integrated system for protemic sample preparation. Hongbo Guo (a technician in Dr. Andrew Emili's Lab) operated and acquired data from HPLC-MS/MS system, as well as contributed in helpful discussions. Victoria Luk helped with carrying out the proteomic protocol. I amalgamated protein precipitation and sample reaction steps into one proteomic protocol, improved the chemistry of certain processing steps allowing for smooth transition from one process to the next and determined a metric for reproducibility of the full proteomic assays. Due to the scope of this work, it continues to be a work-in-progress.

In the work described in the Appendix , Dr. Fiddes and I proposed (and implemented) the idea of using hydrogel discs for a variety of bioassays. Dr. Fiddes and I characterized the design and behavior of hydrogel discs for DMF and developed a fluorogenic assay to characterize movement of molecules through hydrogel discs. Sam Au and Alphonusus Ng, both graduate students, contributed helpful discussions and assisted with development of hydrogel discs for 3D cell culture, and enzyme microreactor, respectively. Victoria Luk contributed high-definition photo images of the DMF-driven hydrogel assays. Dr. Fiddes and I are co-first-authors on a paper describing this work in Biomicrofluidics (Fiddes, L.F., Luk, V.N., Au, S.H., Ng, A.H.C., Luk, V.M., Kumacheva, E., Wheeler, A.R. Hydrogel Discs for Digital Microfluidics Biomicrofluidics in press 2012 DOI: 10.1063/1.3687381.) 
TABLE OF CONTENTS

$\begin{array}{lr}\text { ABSTRACT } & \text { II } \\ \text { ACKNOWLEDGEMENTS } & \text { IV } \\ \text { OVERVIEW OF CHAPTERS } & \mathbf{V} \\ \text { OVERVIEW OF AUTHOR CONTRIBUTIONS } & \text { VII } \\ \text { TABLE OF CONTENTS } & \text { IX } \\ \text { LIST OF TABLES } & \text { XII } \\ \text { LIST OF FIGURES } & \text { XIII } \\ \text { LIST OF ABBREVIATIONS } & \text { XV } \\ \text { LIST OF SYMBOLS } & \text { XVI }\end{array}$

1 CHAPTER 1 • REVIEW OF MICROFLUIDIC TECHNOLOGY FOR CLINICAL PROTEOMIC APPLICATIONS

1.1 Proteomics $\quad 1$

$\begin{array}{ll}\text { 1.1.1 Why is the Study of Proteomics Important } & 1\end{array}$

1.1.2 Tools for Proteomics

1.1.2.1 Two-Dimensional Gel Electrophoresis $\quad 3$

$\begin{array}{lll}1.1 .2 .2 & \text { Shotgun Proteomics } & 4\end{array}$

1.1.2.3 Bottom-up Proteomics $\quad 5$

1.1.2.4 Top-down Proteomics $\quad 5$

$\begin{array}{ll}\text { 1.1.3 Challenges in Proteomics } & 6\end{array}$

1.2 Microfluidics $\quad 6$

$\begin{array}{ll}\text { 1.2.1 Channel Microfluidics } & 7\end{array}$

$\begin{array}{lll}\text { 1.2.1.1 } & \text { Extraction of Proteins in Channel Microfluidics } & 7\end{array}$

1.2.1.2 Proteomic Reactions in Channel Microfluidics 7

1.2.2 Digital Microfluidics $\quad 8$

$\begin{array}{lll}\text { 1.2.2.1 Digital Microfluidics and Proteomics } & 11\end{array}$

\section{CHAPTER 2 • PLURONIC ADDITIVES: A SOLUTION TO STICKY PROBLEMS IN} DIGITAL MICROFLUIDICS

$\begin{array}{lll}2.1 & \text { Introduction } & 12\end{array}$

$2.2 \quad$ Materials and Method $\quad 144$

2.2.1 Reagents and Materials $\quad 14$

2.2.2 Device and Test Substrate Fabrication $\quad 155$

2.2.3 Device Operation 166

2.2.4 Direct Analysis of Protein Adsorption 166

2.2.5 Indirect-Depletion Analysis of Protein Adsorption $\quad 188$

2.2.6 NMR Investigation of Pluronic-Protein Interaction $\quad 18$

$\begin{array}{lll}2.2 .7 & \text { Protein Movability Assay } & 199\end{array}$

$\begin{array}{lll}2.2 .8 & \text { Enzyme Assay } & 19\end{array}$

$\begin{array}{lll}2.3 & \text { Results and Discussion } & 20\end{array}$

$\begin{array}{ll}\text { 2.3.1 Protein Adsorption on DMF Devices } & 20\end{array}$

$\begin{array}{ll}\text { 2.3.2 Worst Case Scenario } & 22\end{array}$

2.3.3 Validation of Results $\quad 25$ 
2.3.4 NMR investigation of Pluronic-Protein Interaction 26

2.3.5 Effects and Mechanism of Pluronic Additives $\quad 27$

$\begin{array}{ll}\text { 2.3.6 Non-Stick Digital Microfluidics } & 29\end{array}$

$\begin{array}{llr}2.4 & \text { Conclusion } & 30\end{array}$

\section{CHAPTER 3 • A DIGITAL MICROFLUIDIC APPROACH TO PROTEOMIC SAMPLE} PROCESSING

$\begin{array}{lll}3.1 & \text { Introduction } & 32\end{array}$

3.2 Materials and Method 33

3.2.1 Reagents and Materials $\quad 33$

3.2.2 Device Fabrication $\quad 34$

3.2.3 Device Operation $\quad 35$

3.2.4 Qualitative Analysis $\quad 35$

3.2.5 Reaction Rate Analysis $\quad 36$

$\begin{array}{lll}3.2 .6 & \text { Reproducibility Assay } & 37\end{array}$

3.3 Results and Discussion $\quad 37$

3.3.1 Multistep Proteomic Processing on DMF Devices 37

3.3.2 Processing Time 41

$\begin{array}{lll}\text { 3.3.3 } & \text { Reproducibility } & 44\end{array}$

$\begin{array}{llr}3.4 & \text { Conclusion } & 45\end{array}$

4 CHAPTER 4 • A TAILORED HYDROGEL MICROREACTOR FOR DIGITAL MICROFLUIDICS: PROTEOMICS APPLICATION 46

$\begin{array}{lll}4.1 & \text { Introduction } & 46\end{array}$

$\begin{array}{lll}4.2 & \text { Materials and Method } & 48\end{array}$

$\begin{array}{lll}\text { 4.2.1 } & \text { Reagents and Material } & 48\end{array}$

$\begin{array}{lll}\text { 4.2.2 } & \text { DMF Device Fabrication and Operation } & 48\end{array}$

$\begin{array}{lll}4.2 .3 & \text { Hydrorogel Disc Fabrication } & 49\end{array}$

4.2.4 Covalent Attachment of Enzymes to Gels 49

4.2.5 Gel Disc Microreactor Optimization $\quad 50$

4.2.6 Proteomic Workup and Mass Spectrometry 51

4.3 Results and Discussion $\quad 53$

4.3.1 Gel Disc Microreactor Formation and Use

4.3.2 Gel Disc Microreactor Optimization $\quad 55$

4.3.3 Comparison of In-Gel and In-Solution Digestion Quality 56

$\begin{array}{ll}\text { 4.3.4 Multiplexed Digestion } & 59\end{array}$

$\begin{array}{llr}4.4 & \text { Conclusion } & 59\end{array}$ 


\section{TOWARDS A FULLY INTEGRATED MICROFLUIDIC SYSTEM FOR FRONT-END PROTEOMIC PROCESSING}

$\begin{array}{lll}5.1 & \text { Introduction } & 61\end{array}$

5.2 Methods and Materials $\quad 62$

5.2.1 Reagents and Materials $\quad 62$

5.2.2 Device Fabrication and Operation $\quad 63$

5.2.3 Workflow of DMF-driven and conventional Proteomic Assay 64

5.2.4 Reversed-Phase Capillary LC-MS/MS Analysis $\quad 65$

5.2.5 Data Analysis $\quad 65$

5.3 Results and Discussion $\quad 66$

5.3.1 Analysis of model serum on DMF device 66

5.3.2 Analysis of model serum on DMF device macroscale processing 69

$\begin{array}{lll}5.4 & \text { Conclusion } & 70\end{array}$

6 CONCLUDING REMARKS AND FUTURE WORK $\quad 71$

APPENDIX 1・HYDROGEL DISCS FOR DIGITAL MICROFLUIDICS

$\begin{array}{lll}\text { A1.1 Introduction } & 74\end{array}$

A1.2 Methods and Materials $\quad 76$

A1.2.1 Reagents and Material $\quad 76$

A1.2.2 DMF Device Fabrication and Operation $\quad 76$

A1.2.3 Formation of Gel Discs $\quad 77$

A1.2.4 Gel Disc Dispensing Measurements $\quad 77$

A1.2.5 Gel Disc Solution Exchange Assay $\quad 77$

A1.2.6 Enzyme Microreactor Assay $\quad 78$

$\begin{array}{ll}\text { A1.2.7 3D Cell Culture } & 79\end{array}$

A1.3 Results and Discussion $\quad \mathbf{8 0}$

A1.3.1 Design and Behaviour of Microgel Discs for Digital Microfluidics $\quad 80$

A1.3.2 Application \#1: Enzyme Microreactor $\quad 85$

A1.3.3 Application \#2: 3D Cell Culture $\quad 86$

$\begin{array}{llc}\text { A1.4 Conclusion } & 88\end{array}$

$\begin{array}{lr}\text { REFERENCES } & 89\end{array}$ 


\section{List of Tables}

\section{Chapter 1}

Table 1.0: Advantages and Disadvantages of Digital Microfluidic Technology

\section{Chapter 2}

Table 2.1: Normalized Protein Adsorption (\% Relative to Control) on DMF Substrates........ 23

Table 2.2: Maximum Movable Protein Concentrations by DMF......................... 29 


\section{List of Figures}

\section{Chapter 1}

Figure 1.1: Proteomic Complexity .................................................. 2

Figure 1.2: Two-dimensional gel electrophoresis versus shotgun proteomics................ 4

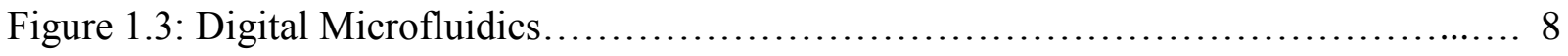

\section{Chapter 2}

Figure 2.1: Protein adsorption from an aqueous droplet onto a DMF device................. 21

Figure 2.2: Confocal analysis of DMF surfaces exposed to labeled proteins................. 24

Figure 2.3: ToF-SIMS spectra of Teflon-coated substrates exposed to labeled proteins........ 26

Figure 2.4: Diffusion of Pluronic 127 with and without BSA............................. 27

Figure 2.5: Digital microfluidic driven proteolysis assay ................................. 31

\section{Chapter 3}

Figure 3.1: A typical proteomic sample processing workup................................ 39

Figure 3.2: MALDI-MS spectra of samples prepared by DMF-driven processing ............ 40

Figure 3.3: Rates of reactions........................................................ 43

Figure 3.4: Experimental precision in DMF-driven assays............................ 44

\section{Chapter 4}

Figure 4.1: Immobilization of enzymes onto hydrogel discs........................... 54

Figure 4.2: Hydrogel proteolytic enzyme microreactors................................. 55

Figure 4.3: Gel disc microreactor optimization........................................... 56

Figure 4.4: Comparison of digestion efficiency..................................................... 58

Figure 4.5: DMF-driven multi-enzyme digestion of a protein sample using hydrogel discs... 60 


\section{Chapter 5}

Figure 5.1: A typical proteomic sample processing workup.................................. 68

Figure 5.2: Spectral count of each analyte recovered from model serum.................. 70

\section{Appendix 1}

Figure A1.1: Images of gel discs on a finger and on a digital microfluidic device............ 81

Figure A1.2: Droplet actuation and hydrogel discs in digital microfluidics................. 82

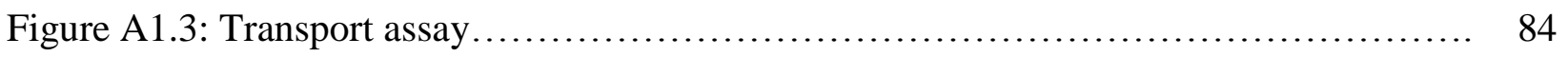

Figure A1.4: Enzyme Microreactor.............................................. 86

Figure A1.5: Three-Dimensional Cell Culture ....................................... 88 


\section{List of Abbreviations}

4-amino-7-aminosulfonylbenzofurazan (ABD-F)

4,4-Difluoro-4-bora-3a,4a-diaza-s-indacene (BODIPY)

Acetonitrile (ACN)

Alpha-cyano-4-hydroxycinnamic acid ( $\alpha$-CHCA)

Bovine serum albumin (BSA)

Deionized water $\left(\mathrm{dH}_{2} \mathrm{O}\right)$

Digital microfluidics (DMF)

Dimethyl sulfoxide (DMSO)

Dithiothreitol (DTT)

Electrospray ionization (ESI)

Electrowetting-on-dielectric (EWOD)

Emerging communications technology institute (ECTI)

Fetal bovine serum (FBS)

Fibrinogen $(\mathrm{Fb})$

Fluorescein isothiocyanate (FITC)

Formic acid (FA)

Fourier-transform ion cyclotron resonance mass spectrometry (FTICR-MS)

Hexamethyldisilazane (HMDS)

Human serum albumin (HSA)

Human Proteome Organization (HuPO)

Indium tin oxide (ITO)

Immobilized-metal affinity chromatography (IMAC)

Immunoglobulin A (IgA)

Immunoglobulin $\mathrm{G}$ (IgG)

Isoelectric point ( $\mathrm{pI}$ )

Mass Spectrometry (MS)

Matrix assisted laser desorption ionization (MALDI)

Peptide Mass Fingerprinting (PMF)

Poly(ethylene glycol) (PEG)

Poly(ethyleneoxide) (PEO)

Poly(propyleneoxide) (PPO)

Post-translation modifications (PTMs)

Pulsed field gradient (PFG)

Region of interest (ROI)

Sinapinic acid (SA)

Stimulated echo (STE)

Sodium dodecyl sulfate (SDS)

Sodium Dodecyl Sulfate Polyacrylamide Gel Electrophoresis (SDS-PAGE)

Solid-phase extraction (SPE)

Tandem MS (MS/MS)

Time-of-Flight Secondary Ion Mass Spectrometry (ToF-SIMS)

Trichloroacetic acid (TCA)

Tris(2-carboxyethyl)phosphine (TCEP)

Two-Dimensional Gel Electrophoresis (2DGE)

Two-Dimensional Liquid Chromatography (2DLC) 


\section{List of Symbols}

$\theta$ contact angle

$\mathrm{V}$ applied voltage

$\theta_{0}$ is the contact angle when the electric field across interfacial layer is zero

$\varepsilon_{\mathrm{r}}$ is the relative permittivity of the dielectric

$\varepsilon_{\mathrm{O}}$ is the permittivity of free space

$\gamma$ is the is the liquid-filler media surface tension

$d$ is the dielectric thickness

$L$ length of the contact line overlapping the actuated electrode

$\mathrm{F}$ driving force on the droplet

$E$ capacitively stored in the system

$f$ frequency

$\varepsilon_{\mathrm{r}, \mathrm{i}, \mathrm{liquid}}$ relative permittivity of liquid

$V_{\mathrm{i}, \text { liquid }}$ voltage drop for the liquid

$\varepsilon_{\mathrm{r}, \mathrm{i}, \mathrm{fill}}$ relative permittivity of filler

$V_{\mathrm{i}, \text { filler }}$ voltage drop for the filler

$d_{i}$ thickness of layer $i$

$f_{c}$ critical frequency

$\gamma_{\mathrm{H}}$ is the magnetogyric ratio of proton

$\delta$ gradient duration

g gradient amplitude

$\Delta$ diffusion time 


\section{Chapter 1 - Review of microfluidic technology for clinical proteomic applications}

\subsection{Proteomics}

The term "proteome" was first coined by Marc Wilkins to describe the total set of proteins expressed in a given cell at a given time ${ }^{1}$ and the study of the proteome is called proteomics. From its first conceptualization in the mid 1990s, ${ }^{2}$ the field of proteomics has undergone a meteoric rise in popularity, leading to the publication of more than 30,000 experimental papers in the field in the past decade. ${ }^{3}$ Here, I discuss why proteomics is important to study, review the common technologies used in proteomic studies, and describe some of the analytical challenges.

\subsubsection{Why is the Study of Proteomics Important}

Proteomics is the field that bridges genomes with cellular behavior. Although genomic biomarkers have been touted as a useful predictive tool that can aid in the diagnosis and prognosis of potential diseases, it is ultimately the gene product (protein) that has an effect cellular function. As depicted in figure 1.1, an organism's genes (static DNA blueprint) are first transcribed into mRNA and then translated into the gene products (proteins). Both mRNA and gene products are dynamic in nature and can undergo myriad modifications. For example, alternative splicing of mRNA can result in the generation of different proteins from the same gene; alternatively, gene products can undergo post-translation modification (i.e., phosphorylation, glycosylation, lipidation, ubiquitination, methylation, acetylation, etc.). These changes drastically affect function of a protein and its effect on a cell; therefore, proteins are a rich source of information regarding the cellular/disease state of an individual.

The field of proteomics encompasses a wide range of sub-disciplines; three major areas include proteome expression profiling, structural proteomics, and functional proteomics. Proteome expression profiling is defined as the identification and quantitation of all proteins in a sample. Expression levels of an entire proteome or subproteomes are compared between samples to identify variations, which may indicate changes in cellular/disease state. Structural proteomics aims to determine the 3D architecture of all proteins, and in addition to identify where the proteins are located within the cell, and to characterize all protein-protein interactions within a protein complex or organelle, creating what is known as a "cell map". 4 


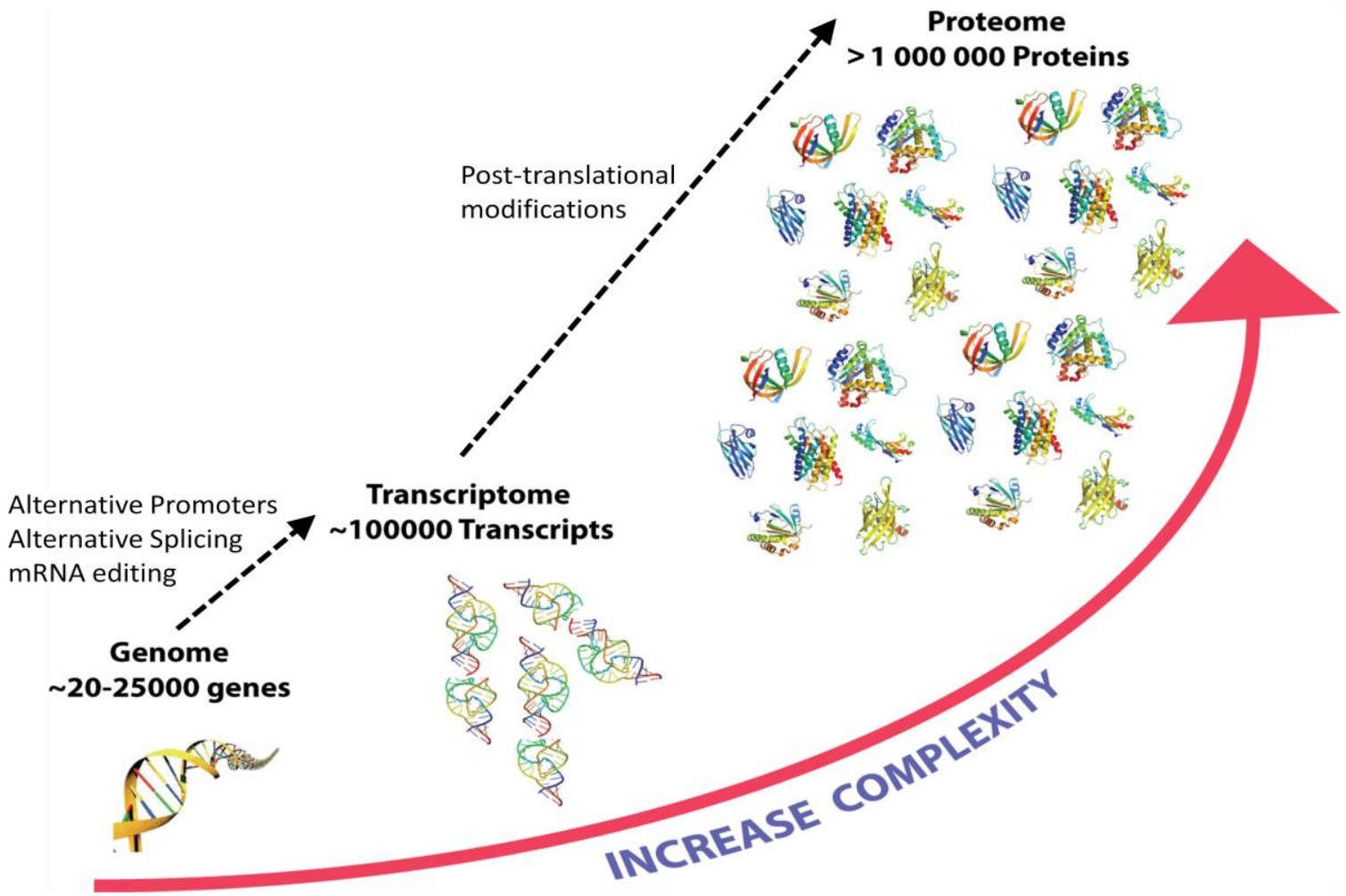

Figure 1.1: Proteome Complexity. Schematic demonstrating the changes involved at the transcriptional and mRNA levels. These modifications exponentially increase the complexity of the proteome relative to both the transcriptome and genome.

Functional proteomics aims to characterize protein activities, multiprotein complexes, and signaling pathways. ${ }^{5}$ Of the three sub-disciplines, proteome expression profiling has received the most attention from corporate and academic researchers; this is likely due to the intimate relationship between disease states and protein expression levels.

Proteome expression profiling studies typically investigate protein expression levels/patterns in abnormal cells (e.g., malignant) in comparison with normal cells. This comparative approach is carried out to identify any proteins that may be upregulated or downregulated in a diseasespecific manner for eventual use as biomarkers of the abnormal state. Various cancer biomarkers are being tentatively identified using the proteome profiling approach, including lung, ${ }^{6,7}$ ovarian, ${ }^{8}$ prostate, ${ }^{9}$ colorectal, ${ }^{10}$ and liver cancers. ${ }^{11}$ It is evident that proteomics is becoming an increasingly important tool for the identification of biomarkers and therapeutic targets for cancer and other diseases. 


\subsubsection{Tools for Proteomics}

Serum is a useful biological sample for biomarker detection because it contains a large number

of proteins, including those released from diseased tissues. ${ }^{12}$ Because of the complexity of the serum proteome, protein mixtures are typically proteolytically digested into peptides of similar size and chemistry making them more suitable for mass spectrometry (MS) analysis. The resultant peptide masses from MS or tandem MS (MS/MS) data are compared to proteomic databases (e.g., UniProtKB/Swiss-Prot) which enable identification by means of peptide mass fingerprinting (PMF) or peptide sequencing, respectively. However, the number of approaches to move from complex protein serum sample to analysis by MS is large, diverse, and growing. Here I review the two standard proteomic techniques, as summarized in figure 1.2; namely, twodimensional gel electrophoresis - mass spectrometry (2DGE-MS) and two-dimensional liquid chromatography - tandem mass spectrometry (2DLC-MS/MS). The advent of more powerful MS instrumentation and new ionization techniques, spawned two different approaches to protein characterization called top-down proteomics and bottom-up proteomics, which are also reviewed in this chapter.

\subsubsection{Two-Dimensional Gel Electrophoresis}

2DGE-MS has historically been the most popular method for proteome profiling. In 2DGE-MS analysis, components of crude protein mixtures (e.g., serum) are separated in two dimensions that are physically orthogonal to each other. In the first dimension, proteins are separated based on charge; specifically, the isoelectric point (pI). In the second dimension, known as sodium dodecyl sulfate polyacrylamide gel electrophoresis (SDS-PAGE), samples are treated with SDS, an anionic detergent that denatures the protein and imparts a negative charge that is proportional to its mass, and allows for separation based on molecular weight. The gels are then fixed and the proteins visualized by staining (e.g., Coomassie Blue or silver staining). The relevant protein bands are then manually excised, destained, washed, reduced, alkylated and digested prior to either electrospray ionization (ESI) or Matrix assisted laser desorption ionization (MALDI) mass spectrometry. These processes (beginning with excision) are then repeated hundreds of times to analyze each relevant spot. It is evident that this technique is highly labour intensive with respect to sample handling and time consuming. It should also be noted that it has limited reproducibility and is biased against certain classes of proteins (e.g., SDS-PAGE has limited compatibility with 
very basic/acidic or hydrophobic proteins), which can reduce the number of proteins detected and make comparison between samples difficult.

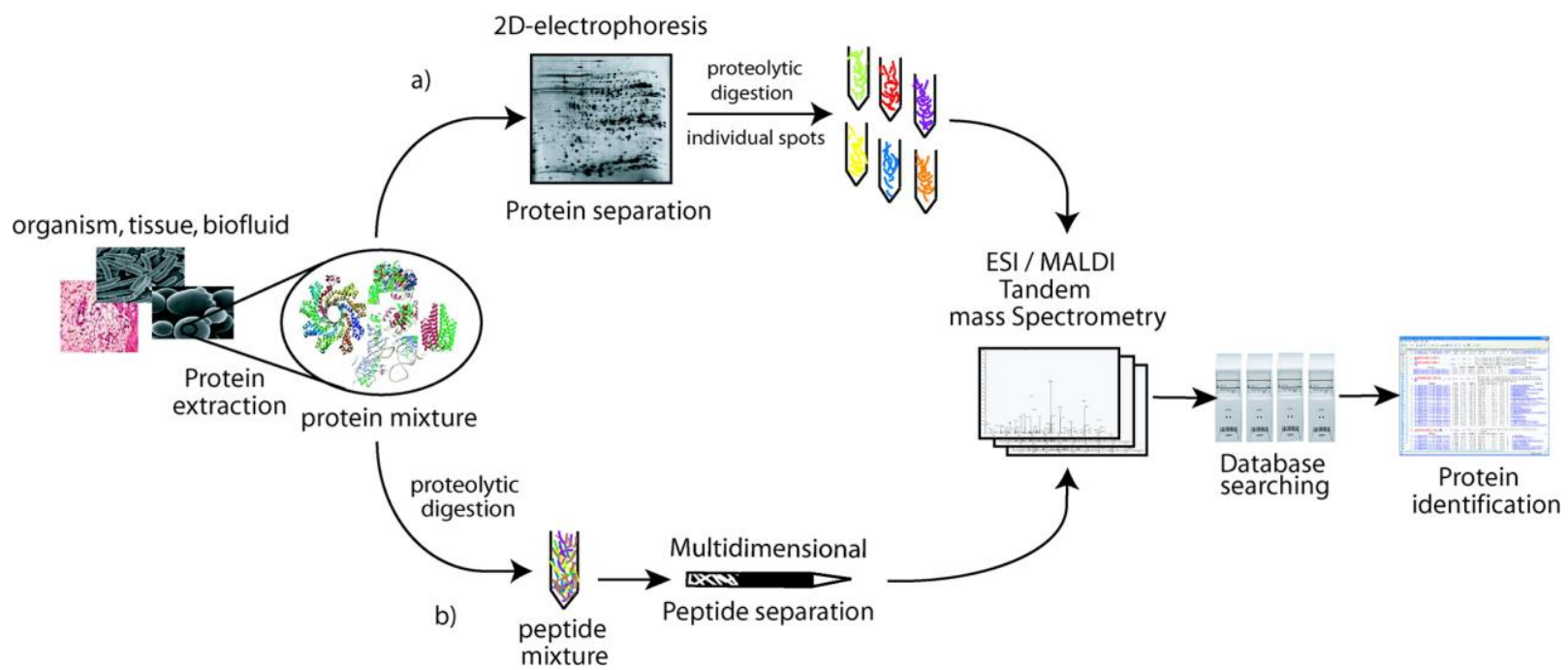

Figure 1.2: Schematic demonstrating (a) two-dimensional gel electrophoresis (2DGE) versus (b) shotgun proteomics for the analysis of proteins. Reproduced from reference ${ }^{228}$ with permission. Copyright $\odot 2007$ American Chemical Society.

\subsubsection{Shotgun Proteomics}

Advances in tandem MS (MS/MS) technology has allowed for the development of alternative techniques for proteome profiling. One common technique is 2DLC-MS/MS or "shotgun proteomics." In this technique, samples are subjected to a series of biochemical processes including alkylation, reduction and digestion, followed by online 2DLC separation. In typical experiments, proteins are first separated on an ion exchange column followed by a reversedphase column and then eluted directly into a MS for detection. Shotgun proteomics is supplanting 2DGE-MS as the most popular method for proteome profiling for two reasons. The first (most obvious) advantage is the elimination of the need for manual excision of protein bands from gels, which is a major bottleneck and source of variability in results. The second advantage is the capacity to analyze the entire sample, not just the visible bands after staining, which diminishes the bias against certain protein classes. Thus, shotgun proteomics represents a significant advancement in the field of proteome profiling, as it allows for high-throughput automated back-end processing (separation and detection). However, front-end processing (serial biochemical processes), a common element in both shotgun proteomics and 2DGE, remains a bottleneck, preventing high-throughput analysis. For example, in a highly cited study ${ }^{13}$ of the 
yeast proteome using shotgun proteomics, prior to analysis, the samples were subjected to a three-day procedure including acidification, denaturing, reduction, alkylation, proteolytic digestion (twice), purification, and dilution prior to analysis by 2DLC-MS/MS.

\subsubsection{Bottom-up Proteomics}

The two techniques for protein characterization described above (section 1.1.2.1. and 1.1.2.2) are the main methods used in the bottom-up proteomics approach, defined as the analysis of peptide fragments derived from digestion, either enzymatically and/or chemically. In general, in this strategy complex protein mixtures are chemically and/or enzymatically digested prior to analysis by MS, followed by identification using established databases. This approach makes it suitable for automation and high-throughput processing ${ }^{14}$ with respect to separation and identification, and is the most common strategy used by the proteomic community.

\subsubsection{Top-down Proteomics}

An alternative strategy for protein characterization that is maturing and growing in popularity is known as Top-down proteomics, which involves the analysis of intact proteins. The primary advantage of this approach compared to bottom-up proteomics is the capacity to identify proteins as distinct molecular species, rather than having to reconstruct the structures from the identity of various peptide fragments. A second advantage is the capacity to identify protein isoforms and post-translation modifications (PTMs) rather than individual peptides that may belong to one or several isoforms. ${ }^{15,16}$ In this approach, proteins from biological samples (e.g., cell lysate) are collected, purified, separated, and then analyzed by MS. The elimination of the protein digestion step allows for isolation of different isoforms prior to MS detection. However, the ability to measure intact proteins requires powerful mass spectrometers with high mass accuracy. Traditionally, this approach has required Fourier-transform ion cyclotron resonance mass spectrometry (FT-ICR-MS or FT-MS). Although FT-ICR-MS is known for its high resolving power and mass accuracy, the expertise required to operate such an instrument, costs associated with maintaining it, complexity of the mass spectra and lack of automation still restricts this

approach from becoming a high throughput method. ${ }^{17}$ An alternative instrument that also has an impact on analytical proteomics is the orbitrap mass analyzer. ${ }^{18}$ It also possesses high mass accuracy and resolution, close to those achievable by the FI-ICR. This mass analyzer is more 
affordable and straight-forward to use compared to the FT-ICR and therefore is becoming more widely adopted for many standard top-down ${ }^{16,19}$ and bottom-up ${ }^{20,21}$ proteomic work.

\subsubsection{Challenges in Proteomics}

It is evident that proteomics is a challenging field to study. For example, the human serum proteome is a very complex biological sample to study because of its large dynamic range (> $\left.10^{10}\right){ }^{22}$ diverse physical (e.g., molecular weight) and chemical properties (e.g., solubility, hydrophobicity, $\mathrm{pH}$ ), low-abundance of target analytes, and presence of interfering compounds (e.g., salts and lipids). In terms of techniques used to characterize proteins there are often limitations with respect to identification of post-translational modification, compatibility/bias of different classes of proteins, limitations in reproducibility, and limitations in automation. Also, many techniques are labour intensive, time consuming and must be performed by well-trained personnel. The complexity of the sample and limited availability of fully automated front-end processing prevents the implementation of anything approaching a high-throughput analysis.

\subsection{Microfluidics}

Microfluidics describes the field of research focused on the properties and applications of fluid flow in micron-dimension lengths in microfabricated devices. Microfluidics has become a powerful tool with the promise to revolutionize chemical and biological analysis. ${ }^{23,24}$ Features $^{2}$ that make microfluidics an attractive technology include reduced sample and reagent consumption, faster reaction times, decreased laboratory footprint, multiplexing capability, increased processing throughput and the potential for portability and automation. These advantages have been touted as being useful for high-throughput proteomic analysis. Here, I review the microfluidic literature with respect to proteomic analysis with specific emphasis on front-end processing. In this case, front-end processing refers to the typical series of biochemical reactions carried prior to separation and MS detection in shotgun proteomics, including: (1) extraction of proteins from heterogeneous samples, and (2) reactions necessary to process samples for analysis by mass spectrometry (reduction, alkylation, and digestion). Currently, there are two paradigms in microfluidics: channel microfluidics (section 1.2.1) and digital microfluidics (section 1.2.2). 


\subsubsection{Channel Microfluidics}

In channel microfluidics, liquids are manipulated in micron dimension channels. Fluid flow in channels can be controlled electrokinetically (electrically driven), ${ }^{25-27}$ hydrodyanamically (pressure driven), ${ }^{28-30}$ or through capillary action. ${ }^{31}$ Based on one or a combination of these methods, fluids can be manipulated in channels in one of two formats, continuous flow or segmented flow. Continuous flow involves the flow of one or more fluid phases through a network of micron-sized channels. Segmented flow (also known as droplet microfluidics) uses two immiscible fluids in channels, including one dispersed phase and one continuous phase. The dispersed phase forms discrete liquid droplets or "plugs" of fluid that are encapsulated in by the carrier fluid.

\subsubsection{Extraction of Proteins in Channel Microfluidics}

Biological samples (e.g., blood, serum, tissue) are very complex, which requires that extraction protocols be used to isolate analytes of interest and remove biological debris that may interfere with downstream processing. In many cases, extraction protocols are implemented in heterogeneous flow systems by incorporating solid-phase components directly into the channels. For example, polystyrene beads ${ }^{32}$ or $\mathrm{C}_{18}$-coated silica beads ${ }^{33}$ have been incorporated into channels as solid-phase extraction (SPE) media to remove salts and other unwanted solution constituents. Alternatively, the extraction of subproteomes has been demonstrated in microflow systems via immobilized metal affinity chromatography (IMAC) beads ${ }^{34}$ or lectin immobilized on polymer monoliths ${ }^{35}$ for the enrichment of phosphopeptides or glycoproteins, respectively. Two-phase continuous flow systems, exploiting the laminar flow phenomenon, have been used for the separation and purification of biological materials such as proteins or cells via liquidliquid extraction. ${ }^{36}$ However, many of the above microfluidics systems are limited to single-step reactions and require fabrication of complicated intricate structures (i.e., weir structures or physical constrictors) to retain solid-phase media.

\subsubsection{Proteomic Reactions in Channel Microfluidics}

Efficient sample preparation is essential for high quality MS protein identification. After protein extraction, sample preparation steps commonly include a series of sequential reactions, including reduction of disulfide bonds, alkylation to prevent the reformation of disulfide bonds, and digestion to cleave the protein into peptides. Several groups have miniaturized and integrated 
sample processing into microfluidic devices in an attempt to solve the problem of laborious, time consuming and irreproducible macroscale sample processing. Many strategies have focused on implementing digestion in microflow systems, in which digestive enzymes (e.g., trypsin) are immobilized in beads, ${ }^{37-39}$ gel matrices, ${ }^{40-43}$ polymer monoliths, ${ }^{44,45}$ membranes, ${ }^{46}$ and others. However, the serial (unidirectional) nature of channel microfluidics has limited such systems to a single step (with some exceptions noted ${ }^{47}$ ). Implementation of multistep reactions with different incubation periods and changes in $\mathrm{pH}$ may be challenging for enclosed microchannels. Although microvalves offers some relief to this problem, ${ }^{48}$ a complicated series of reactions, such as those required for proteome profiling, may still prove to be difficult to carry out in channels.

\subsubsection{Digital Microfluidics}

We propose that an alternative fluid handling technique, called digital microfluidics (DMF), is a good match for implementing multistep sample processing for proteomics (chapter 3). ${ }^{49-52}$ In DMF, discrete microdroplets are manipulated electrostatically on an array of electrodes coated with a hydrophobic, dielectric insulator. ${ }^{53,54}$ Figure 1.3 depicts a schematic of a typical digital microfluidic device. A droplet of polarizable liquid is sandwiched between two planar electrodes in close proximity including (i) a top continuous ground electrode and (ii) an array of independently addressable bottom control electrodes.

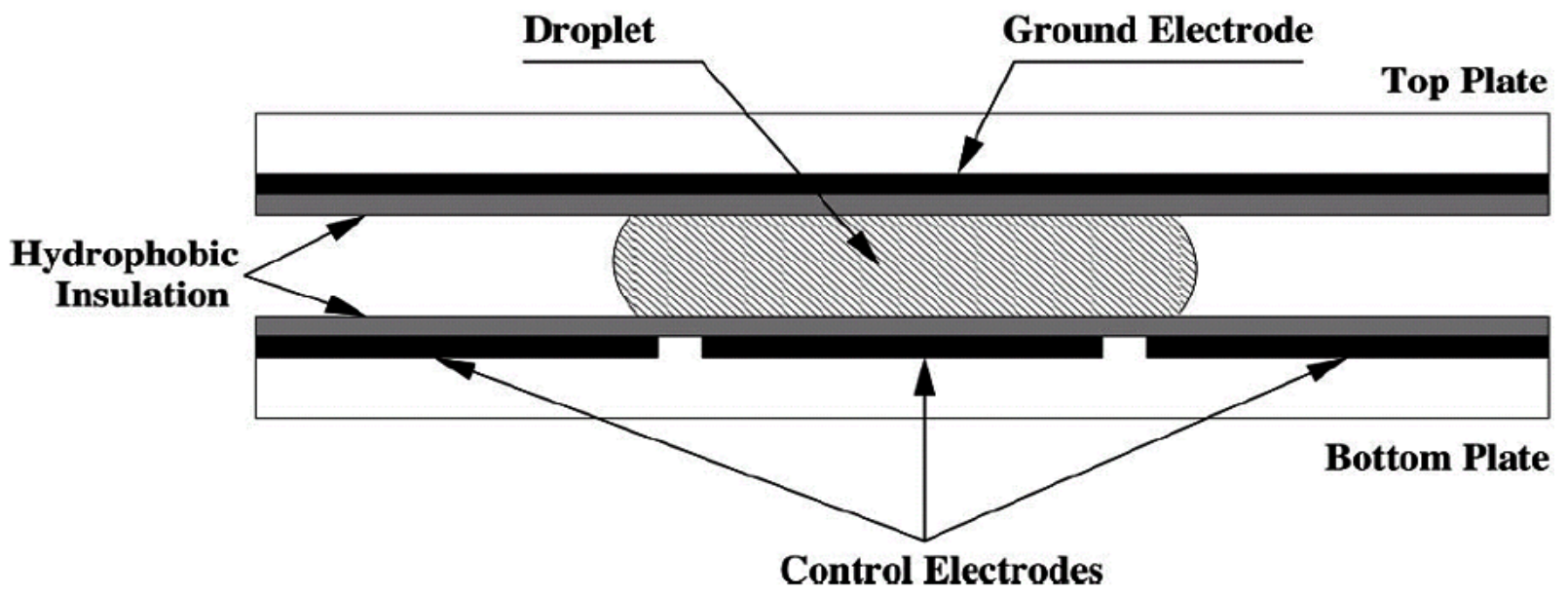

Figure 1.3. Digital Microfluidics. Schematic cross-section of digital microfluidic device. Reproduced from reference ${ }^{53}$ with permission. Copyright $\odot 2000$ American Institute of Physics. 
The classical characterization of droplet movement in DMF is based on a thermodynamic approach using the Young-Lippman equation (Eq 1), ${ }^{55,56}$ where change in contact angle, $\theta$, is a function of applied voltage, V. Here, $\theta_{0}$ is the contact angle when the electric field across interfacial layer is zero, $\varepsilon_{\mathrm{r}}$ is the relative permittivity of the dielectric, $\varepsilon_{\mathrm{o}}$ is the permittivity of free space, $\gamma$ is the is the liquid-filler media surface tension, and $d$ is the dielectric thickness.

$$
\cos \theta=\cos \theta_{0}+\frac{\varepsilon_{0} \varepsilon_{\mathrm{r}} V^{2}}{2 \gamma d}
$$

In this approach, droplet movement is described as being the result of changes in interfacial energy as a result of the accumulation of charge at the surface, ${ }^{57}$ leading to a change in contact angle. Subsequently, this generates a Laplace/capillary pressure due to asymmetric contact angles across the droplet. Upon rearrangement of the above equation (Eq 1) and multiplication by the length of the contact line overlapping the actuated electrode, $L$, it can be seen the driving force on the droplet, $F$ is as follows ${ }^{56}$ :

$$
F=L \gamma_{\mathrm{LG}}\left(\cos \theta-\cos \theta_{0}\right)=\frac{\varepsilon_{0} \varepsilon_{\mathrm{r}} L V^{2}}{2 d}
$$

According to this model, liquid must be conductive and exhibit a large contact angle changes upon application of potential to be movable. ${ }^{58}$ Experiments based on this model were carried out on sessile droplets and the observed phenomenon was named electrowetting-on-dielectric (EWOD). ${ }^{59,60}$ This theory led to many references in the literature of an "EWOD"-based force. However, driving force in DMF is in fact a result of electrostatic forces and large contact angle changes are not a requirement for droplet movement, but rather the wetting is an observable effect of the forces acting on the droplet. ${ }^{61-64}$

A more generalized approach for estimating the force on a droplet is based on an electromechanical derivation. As seen in Eq. 3, the amount of energy, E, capacitively stored in the system is calculated as a function of frequency, $f$ and droplet position along the $\mathrm{x}$-axis, $x .^{57}$

$$
E(f, x)=\frac{L}{2}\left(x \sum_{i} \frac{\varepsilon_{0} \varepsilon_{\text {ri,iliquid }} V_{\mathrm{i}, \text { liquid }}^{2}(f)}{d_{\mathrm{i}}}+(L-x) \sum_{i} \frac{\varepsilon_{0} \varepsilon_{\mathrm{ri}, \mathrm{filler}} V_{\mathrm{i}, \mathrm{ifiller}}^{2}(f)}{d_{\mathrm{i}}}\right)
$$


In Eq. 3., $\varepsilon_{\mathrm{r}, \mathrm{i}, \text { liquid }}, V_{\mathrm{i} \text {,liquid }}$, and $\varepsilon_{\mathrm{r}, \mathrm{i}, \mathrm{fill}}, V_{\mathrm{i}, \text { filler }}$ are the relative permittivity and voltage drop for the liquid and filler fluid portion of the electrode respectively, and $d_{i}$ is the thickness of layer $i$. The $i$ subscript represents one of the dielectric, top and bottom hydrophobic, or liquid/filler layers. The derivative of energy, $E$, with respect to $x$, is the force in the $x$-direction, seen in Eq. 4:

$$
F(f)=\frac{\partial E(f, x)}{\partial x}=\frac{L}{2}\left(\sum_{i} \frac{\varepsilon_{0} \varepsilon_{\mathrm{ri}, \text { liquid }} V_{\mathrm{i}, \mathrm{liquid}}(f)}{d_{\mathrm{i}}}-\sum_{i} \frac{\varepsilon_{0} \varepsilon_{\mathrm{r} \text {.i,filler }} V_{\mathrm{i} \text {,filler }}(f)}{d_{\mathrm{i}}}\right)
$$

A critical frequency, $f_{c}$, can be calculated for each device geometry/liquid combination. ${ }^{64}$ Below this frequency, the estimated force is well described by the thermodynamic (or "EWOD") method. Above this frequency, a significant electric field gradient is established within the droplet resulting in polarization (including in uncharged liquid droplets). A polarizable medium (e.g., water) responds to a nonuniform electric field by collecting preferentially in regions of maximum field intensity resulting in a pulling force towards the activated electrode. This is known as liquid-dielectrophoresis force. ${ }^{61,65}$

A summary of the advantages and disadvantages of digital microfluidic technology is shown in Table 1.0.

Table 1.0. Advantages and Disadvantages of Digital Microfluidic Technology

\begin{tabular}{|c|c|}
\hline Advantages & Disadvantages \\
\hline Individual microreactors & Evaporation \\
\hline Accomadate a wide range of volumes & Biofouling \\
\hline Array geometry & Fabrication \\
\hline Multiplexing & Electronic control system \\
\hline Reconfiguration & Not yet a mature technology \\
\hline
\end{tabular}




\subsubsection{Digital Microfluidics and Proteomics}

When I began my thesis work, digital microfluidics had only been used for a few applications related to proteomics, focused on substrate preparation for analysis by MALDI-MS. ${ }^{66-68}$ In the work described in this dissertation, I took on the challenge of applying digital microfluidics to enable reproducible sample processing for proteomic applications. This work required the development of a strategy to inhibit protein biofouling in DMF devices (chapter 2), techniques to implement standard biochemical reactions involved in proteomic sample preparation (chapters 3 and 4), and the integration of biochemical reactions with protein extraction from complex mixtures (chapter 5). Along the way, I developed the first techniques combining digital microfluidics with hydrogels (chapter 4), and applied these techniques to several applications outside of proteomics (Appendix 1). In all, this thesis represents an important step towards the goal of developing an integrated proteome profiling platform using microfluidics. 


\section{Chapter 2 - Pluronic Additives: A Solution to Sticky Problems in Digital Microfluidics}

Digital microfluidics (DMF) is a promising technique for carrying out miniaturized, automated biochemical assays in which discrete droplets of reagents are actuated on the surface of an array of electrodes. A limitation for DMF is nonspecific protein adsorption to device surfaces, which interferes with assay fidelity and can cause droplets to become unmovable. Here, we report the results of a quantitative analysis of protein adsorption on DMF devices by means of confocal microscopy and secondary ion mass spectrometry. This study led us to a simple and effective method for limiting the extent of protein adsorption: the use of low concentrations of Pluronic F127 as a solution additive. This strategy has a transformative effect on digital microfluidics, facilitating the actuation of droplets containing greater than 1000-fold higher protein concentrations than is possible without the additive. To illustrate the benefits of this new method, we implemented a DMF-driven protein digest assay using large concentrations $(1 \mathrm{mg} / \mathrm{mL})$ of protein-substrate. The use of Pluronic additives solves a sticky problem in DMF, which greatly expands the range of applications that are compatible with this promising technology.

\subsection{Introduction}

A new paradigm for miniaturized bioassays has recently emerged and is called "digital" (or droplet-based) microfluidics. In digital microfluidics (DMF), droplets containing samples, reagents, and other liquids are manipulated on the surface of an array of electrodes by means of electrowetting ${ }^{53,54}$ and/or dielectrophoresis. ${ }^{63,69}$ By applying a sequence of potentials to adjacent electrodes, a droplet of fluid can be dispensed from a reservoir, transported on the array, and merged with other droplets to implement nanoliter-scale reactions. Because each droplet is isolated from its surroundings rather than being embedded in a stream of fluid (as is the case for microchannels), DMF is a facile method for forming microreactors in which there is no possibility that reagents or samples will diffuse away. Perhaps most importantly, because DMF is implemented in an array geometry, it seems a natural fit for parallel-scale, multiplexed analyses. ${ }^{70}$ As the popularity of this technique grows, it is being applied to an ever-expanding range of applications, including cell-based assays, ${ }^{71}$ enzyme assays, ${ }^{72-76}$ protein profiling, ${ }^{66-68}$ and the polymerase chain reaction. ${ }^{77}$ 
Unfortunately, digital microfluidics is limited by a critical problem: nonspecific adsorption of biomolecules to device surfaces, or biofouling. DMF surfaces are typically formed from a hydrophobic material such as Teflon-AF, and although Teflon is marketed as a "non-stick" surface, it is actually quite prone to biofouling because of its hydrophobicity ${ }^{78,79}$ Teflon is not unique. In fact, most low-energy surfaces become fouled when exposed to solutes possessing nonpolar residues in aqueous solutions. This causes analytical problems for many applications (biosensors, medical implants, pipet tips, centrifuge tubes, etc.): molecules that adsorb to surfaces have reduced activities, become lost when samples are transferred to other vessels, and desorb unpredictably, leading to cross-contamination. Digital microfluidics also suffers from these analytical problems but in addition suffers from a mechanical problem: in DMF, droplet actuation is dependent upon smooth, homogeneous surfaces, and when appreciable biofouling occurs, droplets become stuck, rendering devices useless. ${ }^{80-82}$ Clearly, if DMF is going to mature from being a technology for aficionados to being a technique that is useful for biochemical applications at large, the problem of biofouling must be overcome. Here, we present a quantitative evaluation of biofouling on digital microfluidic devices. Our primary tool was confocal microscopy. In assays similar to those reported recently by Tserepi et al., ${ }^{80}$ droplets containing labeled proteins were manipulated by DMF, and the extent of protein adsorption was characterized by fluorescence (Figure 2.1). This technique was versatile and robust and allowed us to evaluate several experimental conditions to develop a new strategy for preventing protein adsorption. To validate these results, we used an orthogonal technique, secondary ion mass spectrometry (SIMS).

We note that this is not the first attempt to characterize and/or prevent biofouling in digital microfluidics. Previous efforts have included the use of water-immiscible oils, ${ }^{73,75}$ the application of specific $\mathrm{pH}$ and voltage biases, ${ }^{81}$ and the formation of textured device surfaces. ${ }^{82-84}$ In the first method, ${ }^{73,75}$ aqueous droplets are manipulated through a matrix of oil (rather than air, as they are in our work), which functions to encapsulate the droplets, preventing contact with device surfaces. Although this technique is effective at reducing surface adsorption, it has several disadvantages, including incompatibility with miscible solvents such as ethanol or methanol, and partitioning of nonpolar analytes from aqueous droplets into the oil matrix. In the second method, ${ }^{81}$ actuation parameters including voltage bias and $\mathrm{pH}$ are carefully tuned to limit the electrostatic attraction of proteins to device surfaces; however, this method does not prevent 
hydrophobic interactions, and it is unlikely to be useful for assays requiring reagents and solutions with different $\mathrm{pI}$ and $\mathrm{pH}$ values. The third method ${ }^{82-84}$ may be the most promising because micro- or nanostructured device surfaces have reduced contact areas with aqueous droplets, which limits the amount of molecular adsorption. Thus far, however, such surfaces have proven difficult to integrate with DMF actuation because droplets tend to wet the interfeature regions, causing them to become stuck. ${ }^{84}$ We assert that whereas these previous techniques to reduce biofouling in DMF may be useful in some circumstances, none of them represents a universal solution for the wide range of applications envisioned for this promising technology.

Here, we introduce a new method for minimizing the extent of biofouling in digital microfluidics. In this work, we have built on the extensive literature ${ }^{85-87}$ regarding the use of poly(ethyleneoxide) (PEO), also known as poly(ethylene glycol) (PEG), to reduce the surface adsorption of proteins and other solutes. Specifically, we evaluated the efficacy of PEOcontaining triblock copolymers distributed under the trade name Pluronic (BASF, Florham Park, NJ). Whereas various kinds of Pluronics have been used for applications in microchannels, ${ }^{88,89}$ we believe this to be the first report of the pairing of Pluronics and digital microfluidics. Because DMF is mechanically dependent upon device surface homogeneity (as described above), the Pluronic based method reported here has a transformative effect on the technique, facilitating the actuation of droplets containing greater than 1000-fold higher protein concentrations than is possible for conventional DMF. This technique has the potential to be a near universal solution for preventing biomolecular adsorption in digital microfluidics, thus greatly expanding the range of applications compatible with this promising technique.

\subsection{Materials and Method}

\subsubsection{Reagents and Materials}

Pluronic F68, Pluronic F127, Fluorinert FC-40, Tris-HCl, sodium azide, bovine serum albumin (BSA), fibrinogen $(\mathrm{Fb})$, casein, and FITC-labeled casein (from bovine milk) were purchased from Sigma Chemical (Oakville, ON). Fetal bovine serum (FBS), FITC-BSA, Alexa Fluorlabeled fibrinogen from human plasma, and an E6638 EnzChek protease assay kit were 
purchased from Invitrogen (Burlington, ON). Parylene-C dimer was from Specialty Coating Systems (Indianapolis, IN), and Teflon-AF was from DuPont (Wilmington, DE).

Stock solutions $(5.0-60 \mathrm{mg} / \mathrm{mL})$ of all lyophilized proteins were prepared in $10 \mathrm{mM}$ Tris- $\mathrm{HCl}$ (pH 7.8) buffer containing $0.1 \mathrm{mM}$ sodium azide. Stock solutions of Pluronic F68 and F127 were formed in Tris- $\mathrm{HCl} /$ sodium azide buffer $(1.0 \% \mathrm{w} / \mathrm{v})$, and in $\mathrm{dH}_{2} \mathrm{O}$ with no buffer $(2.0 \% \mathrm{w} / \mathrm{v})$. For quantitative protein adsorption measurements, working protein solutions were formed by diluting stock solutions to $1.0 \mathrm{mg} / \mathrm{mL}$ protein with $x \% \mathrm{w} / \mathrm{v}$ Pluronic F68 or F127 ( $x=0,0.08,0.4,0.8)$. For indirect-depletion measurements, working solutions were formed by diluting labeled BSA to concentrations ranging from 0.92 to $1.0 \mathrm{mg} / \mathrm{mL}$ in $10 \mathrm{mM}$ Tris- $\mathrm{HCl}$ buffer. For DMF movability assays, unlabeled proteins were diluted to various concentrations in Tris- $\mathrm{HCl}$ buffer.

Clean-room reagents and supplies included Shipley S1811 photoresist and MF321 developer from Rohm and Haas (Marlbor- ough, MA), AZ300T photoresist stripper from AZ Electronic Materials (Somerville, NJ), solid chromium and gold from Kurt J. Lesker Canada (Toronto,ON), CR-4 chromium etchant from Cyantek (Fremont, CA), hexamethyldisilazane (HMDS) from Shin-Etsu MicroSi (Phoenix, AZ), and concentrated sulfuric acid and hydrogen peroxide (30\%) from Fisher ScientificCanada (Ottawa, ON). Piranha solution was prepared as a 3:1 v/v mixture of sulfuric acid/hydrogen peroxide.

\subsubsection{Device and Test Substrate Fabrication}

Digital microfluidic devices were formed using conventional methods in the University of Toronto Emerging Communications Technology Institute (ECTI) fabrication facility. Glass wafers were cleaned in piranha solution $(10 \mathrm{~min})$ and then coated with chromium $(60 \mathrm{~nm})$ and gold $(120 \mathrm{~nm})$ by electron beam deposition. After rinsing and baking on a hot plate $\left(115^{\circ} \mathrm{C}, 5\right.$ min), the substrates were primed by spin coating with HMDS (3000 rpm, $30 \mathrm{~s}$ ) and were then spin coated with Shipley S1811 photoresist (3000 rpm, $30 \mathrm{~s}$ ). Substrates were prebaked on a hotplate $\left(100^{\circ} \mathrm{C}, 2 \mathrm{~min}\right)$ and exposed through a photomask using a Suss Mikrotek mask aligner. Substrates were developed in MF321 (3 min) and then postbaked on a hot plate $\left(100^{\circ} \mathrm{C}, 1 \mathrm{~min}\right)$. After photolithography, substrates were immersed in gold etchant (50 s), followed by chromium etchant (30 s). Finally, the remaining photoresist was stripped in AZ300T (10 min). 
After forming electrodes, devices were coated with parylene-C $(2 \mu \mathrm{m})$ and Teflon-AF (50 nm). Parylene $\mathrm{C}$ was applied using a vapor deposition instrument (Specialty Coating Systems), and Teflon-AF was spin coated (1\% w/w in Fluorinert FC-40, 2000 rpm, $60 \mathrm{~s}$ ) and then postbaked on a hotplate $\left(160^{\circ} \mathrm{C}, 10 \mathrm{~min}\right)$. To facilitate the application of driving potentials, the polymer coatings were locally removed from the contact pads by gentle scraping with a scalpel or the tip of a voltage probe. In addition to patterned devices, unpatterned indium tin oxide (ITO)-coated glass substrates (Delta Technologies Ltd., Stillwater, MN) were spin coated with Teflon-AF (50 $\mathrm{nm}$, as above).

Unpatterned test substrates used to mimic DMF devices were prepared by cleaning glass slides in piranha solution (10 min), followed by coating with parylene-C $(2 \mu \mathrm{m})$ and Teflon-AF (50 $\mathrm{nm})$, as above.

\subsubsection{Device Operation}

Devices were assembled with an unpatterned ITO/glass top plate and a patterned bottom plate separated by a spacer formed from one piece of double-sided tape ( $70 \mu \mathrm{m}$ thick). As described

previously, ${ }^{53}$ droplets were sandwiched between the two plates and actuated by applying electric potentials between the top electrode and sequential electrodes on the bottom plate. The driving potentials of $60-80 \mathrm{~V}_{\text {rms }}$ were generated by amplifying the output of a function generator operating at $18 \mathrm{kHz}$ and were applied manually to exposed contact pads on the bottom plate surface. Droplet actuation was monitored and recorded by a CCD camera mounted on a stereomicroscope with fluorescence imaging capability (Olympus Canada, Markham, ON). The devices used here had a $4 \times 6$ array of $1 \mathrm{~mm} \times 1 \mathrm{~mm}$ actuation electrodes, with interelectrode gaps of $5-40 \mu \mathrm{m}$.

\subsubsection{Direct Analysis of Protein Adsorption}

Protein adsorption was assayed on two kinds of substrates: DMF devices and unpatterned test substrates. For the former, droplets containing labeled proteins were manipulated by DMF, after which the top plate and droplet were removed and the device was stored in the dark until analysis. For the latter, $3 \mu \mathrm{L}$ droplets of protein working solutions were pipetted onto test substrates, forming circular footprints (radius $\sim 1 \mathrm{~mm}$ ) on the surface. At least six replicates were 
evaluated for each experimental condition. Substrates held $\sim 28$ droplets, including at least 1 background sample (containing no protein) for each unique condition (intra-device). Adsorption was allowed to proceed for $45 \mathrm{~min}$ in a humidified chamber (a Petri dish partially filled with $\mathrm{dH}_{2} \mathrm{O}$ ), after which the substrates were immersed in $\mathrm{dH}_{2} \mathrm{O}$ with gentle agitation for 10 min to remove nonadsorbed proteins (dilution factor $\sim 10$ 000). After rinsing, samples were air dried and stored in the dark until further analysis. In some experiments, substrates were prepassivated with Pluronic prior to exposure to proteins. For these experiments, substrates were immersed in $2 \% \mathrm{w} / \mathrm{v} \mathrm{F} 68$ or $\mathrm{F} 127$ in $\mathrm{dH}_{2} \mathrm{O}$ (10 min) and then air dried (with no rinse) immediately before protein adsorption. All assays involving Pluronics were carried out at room temperature (20 $\left.25^{\circ} \mathrm{C}\right)$.

Confocal microscopy was the primary tool used to evaluate protein adsorption on surfaces. In these experiments, a Fluoview 300 scanning confocal microscope (Olympus, Markam, ON) equipped with an $\mathrm{Ar}^{+}$(488 nm) laser was used, in conjunction with a 100× objective (NA 0.95) for the analysis of proteins adsorbed to DMF device surfaces (Figure 2.1) or a 4x objective (NA 0.45) for the analysis of proteins adsorbed to test substrates (Figure 2.2). Fluorescence from adsorbed FITC and Alexa Fluor-488-labeled proteins was passed through a 510 - 525 nm bandpass filter, and each digital image was formed from the average of four frames using FluoView image acquisition software (Olympus). In all cases, experimental parameters were optimized such that the PMT was not saturated with signal from the most fluorescent samples. No attempt was made to quantify the density or numbers of molecules adsorbing to surfaces, thus nonlinear effects such as quenching and surface saturation were not considered.

For comparative analysis, images were processed using ImageJ (US National Institutes of Health, http://rsb.info.nih.gov/ij/). In each image, a circular (radius $1 \mathrm{~mm}$ ) region of interest (ROI) was drawn around the fluorescent spot. For background samples and others for which no or low signal was observed, the ROI was arbitrarily defined in the center of the image. In each ROI, the fluorescence was quantified by integrating the brightness values over all pixels. All measurements were then background corrected by subtracting the integrated value collected from the corresponding background ROI. Replicate measurements were averaged and normalized relative to control measurements of labeled proteins containing no Pluronics. Two-tailed $t$-tests assuming equal variances for each experimental condition were used to determine significance. 
Secondary ion mass spectrometry (SIMS) was used as an orthogonal method to validate the results obtained by confocal microscopy. These experiments made use of a ToF-SIMS IV instrument (ION-TOF, Münster, Germany) at the Surface Interface Ontario facility located at the University of Toronto. Test substrates with adsorbed protein samples were probed by collecting negative ion spectra in static SIMS mode ${ }^{90}$ (primary ion dose $<10^{13} \mathrm{~cm}^{-2}$ ) using a $25 \mathrm{keV} \mathrm{Ga}^{+}$ primary ion gun and a raster beam area of $500 \mu \mathrm{m} \times 500 \mu \mathrm{m}$ (residence time $30 \mathrm{~s}$ ). Charge neutralization was achieved using the electron flood gun associated with the instrument. SIMS images were processed using ION-TOF software and facilitated the comparison of multiple spectra, targeting selected $\mathrm{m} / \mathrm{z}$ ratios.

\subsubsection{Indirect-Depletion Analysis of Protein Adsorption}

An indirect depletion method was used to evaluate the amount of protein that adsorbs to DMF devices from aqueous droplets. In this analysis, aqueous droplets containing FITC-casein at various concentrations $(1.00,0.98,0.96,0.94,0.92$, and $0.90 \mathrm{mg} / \mathrm{mL})$ were depleted by pipetting them onto test substrates (five replicates at each concentration). After incubation (45 min) in a humidified chamber, these depleted droplets were recollected into a pipet, deposited into a well in a multiwell plate, and diluted to $300 \mu \mathrm{L}$ with buffer. A PHERAstar multiwell plate reader (BMG Labtech, Durham, NC) was used to measure the fluorescence $\left(\lambda_{\mathrm{ex}}=485 \mathrm{~nm} ; \lambda_{\mathrm{em}}=520\right.$ $\mathrm{nm}$ ) of droplets that were depleted on substrates (experimental) as well as droplets (five replicates at $1.00 \mathrm{mg} / \mathrm{mL}$ ) that were directly dispensed into the multiwell plate, repipetted and dispensed again, and then diluted to $300 \mu \mathrm{L}$ with buffer (control). This extra pipet step was included to ensure that all samples (experimental and control) were handled with the same number of pipet steps. Two-tailed $t$ tests assuming equal variances for each experimental condition were used to determine significance.

\subsubsection{NMR Investigation of Pluronic-Protein Interaction}

NMR spectra were recorded on an Infinity $500 \mathrm{MHz}$ NMR spectrometer (Varian Inc., Palo Alto, CA) using a $5 \mathrm{~mm}$ double resonance liquid probe at $25 \pm 0.5^{\circ} \mathrm{C}$. Sample solution concentrations were $0.08 \%$ w/v F127 in TrisHCl buffer, with and without $1 \mathrm{mg} / \mathrm{mL}$ BSA. ${ }^{1} \mathrm{H}$ NMR diffusion measurements were performed at $499.78 \mathrm{MHz}$ using the stimulated echo (STE) pulsed field gradient (PFG) procedure, with $5 \mathrm{~ms}$ gradient pulse duration and variable gradient pulse 
amplitude $(0.595,0.655,0.714,0.774,0.833,0.893,0.952,1.012,1.071,1.131,1.190,1.250$, 1.309, 1.369, 1.428, 1.488, 1.547, 1.607, 1.666, $1.726 \mathrm{~T} / \mathrm{m})$. The field gradient pulses were applied along the longitudinal $(z)$ direction exclusively. Typical acquisition parameters were: a $90^{\circ}$ pulse length of $16 \mu \mathrm{s}$, a spin echo delay of $10 \mathrm{~ms}$, a recycle delay of $5 \mathrm{~s}$, a spectral width of $10 \mathrm{kHz}$ and a 4000 point data set. The radio frequency pulses were cycled according to the procedure outlined by Fauth et al. ${ }^{91}$ to remove unwanted echoes. For each gradient amplitude, 16 scans were acquired for sufficient $\mathrm{S} / \mathrm{N}(>10: 1)$. Spectra were processed with an exponential multiplication equivalent to $5 \mathrm{~Hz}$ line broadening prior to Fourier transformation, and were referenced to tetramethylsilane (TMS). Gradient strength was calibrated from the known diffusion coefficient of deuterated water (HDO) at $25^{\circ} \mathrm{C}$. The ${ }^{1} \mathrm{H}$ resonance for PEO units in F127 was assigned as $3.3 \mathrm{ppm}$.

\subsubsection{Protein Movability Assay}

The relationship between protein concentration and movability by DMF was probed using solutions of unlabeled proteins (Casein, BSA, and Fb) as well as fetal bovine serum. Droplets containing various concentrations of these test solutions with and without Pluronics $(0.08 \% \mathrm{w} / \mathrm{v}$ F127) were assayed for movability, which is defined as the capacity to translate a droplet across a series of four electrodes, back and forth twice (i.e., a total of 16 electrode-to-electrode steps).

Driving potentials were typically $\sim 60 \mathrm{~V}$ (enough for contact angle change saturation ${ }^{92}$ ), but when a given droplet resisted movement, the driving potential was increased until either (a) the droplet moved or (b) the device experienced dielectric breakdown (i.e., became unusable). At least three replicates of each experimental condition were evaluated on three separate devices to account for interdevice variation, and the maximum movable concentration for each protein was recorded.

\subsubsection{Enzyme Assay}

Stock solutions of $100 \mu \mathrm{g} / \mathrm{mL}$ trypsin and $1 \mathrm{mg} / \mathrm{mL}$ labeled, quenched bodipy-casein (each with $0.08 \%$ w/v F127 additive) were prepared using the E6638 EnzChek Protease Assay Kit. For microscopy, $70 \mathrm{~nL}$ droplets of each stock solution were actively dispensed from separate reservoirs onto the electrode array and then merged and mixed by moving the coalesced droplet around a loop of six actuation electrodes. For quantitative analysis, the procedure was replicated 
at lower concentrations of protein $(10 \mu \mathrm{g} / \mathrm{mL}$ trypsin, $2.5 \mu \mathrm{g} / \mathrm{mL}$ bodipy-casein, both with $0.08 \%$ F127), and after mixing, the device was positioned on the top of a microtiter plate and inserted into a PheraStar multiwell plate reader for fluorescence detection $\left(\lambda_{\mathrm{ex}}=485 \mathrm{~nm}, \lambda_{\mathrm{em}}=520 \mathrm{~nm}\right.$, focal height $=15.0 \mathrm{~mm}$, gain $=900)$. The fluorescence from the merged droplet was measured in intervals of $60 \mathrm{~s}$ for $28 \mathrm{~min}$. Four replicate trials were conducted.

\subsection{Results and Discussion}

\subsubsection{Protein Adsorption on DMF Devices}

Digital microfluidic device surfaces are typically formed from Teflon-AF, which is prone to unwanted protein adsorption from aqueous solutions. As a consequence, DMF applications involving proteins suffer from analytical problems (i.e., loss of analyte, cross-contamination, etc.) and mechanical problems when enough protein adsorbs to a DMF device surface, droplets become stuck, rendering the device useless. This phenomenon is illustrated in Figure $2.1-\mathrm{a}$ droplet containing FITC-BSA $(5 \mu \mathrm{g} / \mathrm{mL})$ was manipulated between four electrodes until the droplet became stuck (which occurred after 16 electrode-to-electrode steps). As shown, the FITC-BSA that adsorbed to the surface of the device as a consequence of the droplet movement was detectable by confocal microscopy. This result (i.e., a detectable amount of protein) is fortunate because it allowed us to conduct comparative experiments evaluating conditions for reducing the level of biofouling (described in the following section, section 2.3.3).

In contrast to confocal microscopy, an indirect-depletion method assaying the concentration of protein in solution after exposure to a Teflon-coated substrate (described in the section 2.2.5) was not sensitive enough to detect the depleted protein. For example, a $t$-test could distinguish between the fluorescence from standard solutions of 0.92 and $1.00 \mathrm{mg} / \mathrm{mL}$ FITC-casein $(\mathrm{p}<$ 0.05) but could not distinguish between $1.00 \mathrm{mg} / \mathrm{mL}$ FITC-casein and an identical sample that had been predepleted on a Teflon surface $(\mathrm{p}=0.18)$. This result is encouraging for those who wish to use DMF to develop analytical methodologies involving proteins because it suggests that the number of molecules in solution that adsorb to a DMF device surface is low (i.e., less than 8\%). But clearly, the amount that does adsorb causes mechanical problems because droplets become stuck and can no longer be moved. In the following sections, we describe our efforts to characterize this phenomenon and develop strategies to minimize it. 


\section{BEFORE movement}

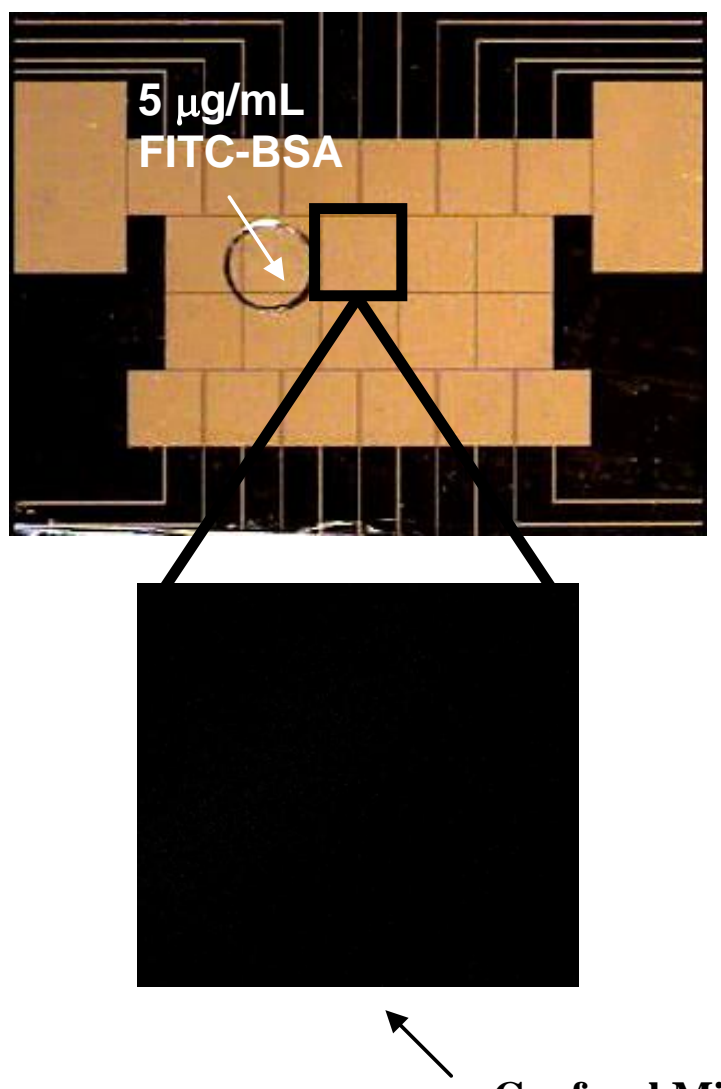

\section{AFTER movement}

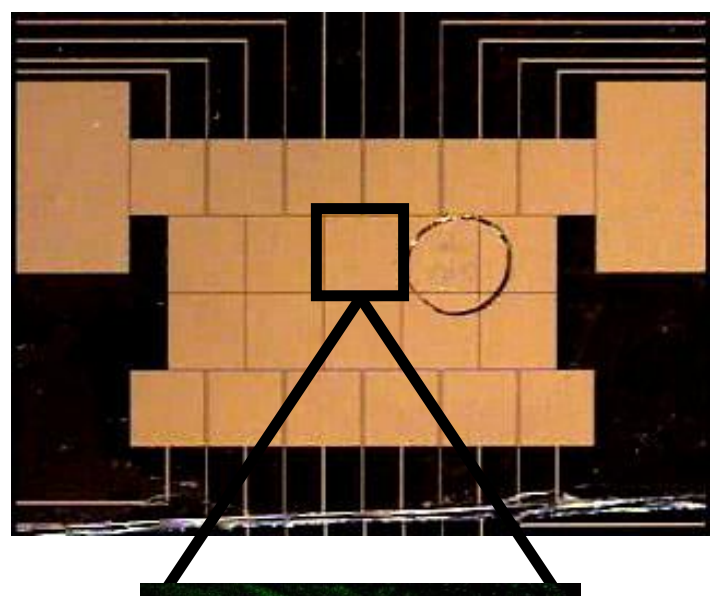

\section{Confocal Microscopy Images}

Figure 2.1: Protein adsorption from an aqueous droplet onto a DMF device. The left image shows a device prior to droplet actuation, paired with a corresponding confocal image of a central electrode. The right image shows the same device after a droplet containing FITC-BSA $(5 \mu \mathrm{g} / \mathrm{mL})$ has been cycled over the electrode 4 times, paired with a confocal image collected after droplet movement. The two images were processed identically to illustrate that confocal microscopy can be used to detect the protein that adsorbs to a device as a result of droplet actuation. 


\subsubsection{Worst Case Scenario}

One strategy that might be used to reduce the effects of biofouling would be to translate droplets very rapidly to limit the amount of adsorption to any given electrode. (DMF is capable of actuation at $25 \mathrm{~cm} / \mathrm{s}^{93}$ ) Unfortunately, this strategy is untenable for many applications that require that reagents be merged and incubated in a static (i.e., not moving) droplet for a period of time to allow a reaction to complete. With this in mind, we developed an assay to estimate the relative amount of protein that adsorbs to a Teflon-coated surface in a worst case scenario in which a droplet remains static on one spot for $45 \mathrm{~min}$. Surfaces were exposed to droplets containing fluorescently labeled casein, BSA, or Fb and were then rinsed, dried, and evaluated by confocal fluorescence microscopy.

In this worst case scenario analysis, two strategies for reducing the extent of protein adsorption were investigated: (1) protein samples were spiked with Pluronics as additives in different concentrations $(0.08,0.4$, and/or $0.8 \% \mathrm{w} / \mathrm{v})$, and (2) devices were prepassivated with surfaceadsorbed Pluronics (a strategy that has proven useful for reducing nonspecific adsorption for other applications ${ }^{87,94}$ ). For both strategies, two different kinds of Pluronics, F68 and F127 (with $\mathrm{PEO}_{\mathrm{x}} / \mathrm{PPO}_{\mathrm{y}} / \mathrm{PEO}_{\mathrm{z}}$ molecular ratios of 76:30:76 and 99:67:99, respectively), were evaluated. The critical micellar concentrations (CMCs) for F68 and F127 are approximately $10 \mathrm{~g} / \mathrm{dL}(0.1 \% \mathrm{w} / \mathrm{v}$ at $\left.20^{\circ} \mathrm{C}\right)^{95}$ and $2.5 \mathrm{~g} / \mathrm{dL}\left(0.025 \% \mathrm{w} / \mathrm{v}\right.$ at $\left.25^{\circ} \mathrm{C}\right),{ }^{96}$ respectively; thus, all solutions used in strategy 1 contained micelles. Additionally, F127 is known to form gels at high concentrations and temperatures ${ }^{97-99}$ (i.e., $15-30 \% \mathrm{w} / \mathrm{v}, 37^{\circ} \mathrm{C}$ ); thus, the parameters in our experiments were well below those required for gelation.

Figure 2.2a shows typical images collected in a confocal microscopy assay for a surface exposed to labeled protein under the worst case scenario conditions. As shown in the left panel, in an area exposed to a droplet containing only labeled protein, a circular fluorescent feature was observed that corresponded to the droplet footprint. In contrast, in an area exposed to a droplet containing labeled protein and $0.08 \% \mathrm{w} / \mathrm{v}$ F127, very little fluorescence was observed. As shown in Table 2.1, all of the pluronic-additive treatments (strategy 1, above) reduced the level of protein adsorption to surfaces. Each condition resulted in a > 99\% reduction in signal from adsorbed $\mathrm{BSA}$ and Fb, but F127 was significantly more effective at reducing the adsorption of casein, which suggests that F127 may be a more widely applicable adsorption blocker. There are small 
but significant differences ${ }^{100}$ in the effectiveness of the different concentrations of F127. The results for one case, $0.08 \%$ F127, are shown graphically in Figure 2.2b.

Table 2.1. Normalized Protein Adsorption (\% Relative to Control) on DMF Substrates ${ }^{a}$

\begin{tabular}{|c|c|c|c|c|c|c|c|}
\cline { 2 - 7 } \multicolumn{1}{c|}{} & \multicolumn{3}{c|}{$\%$} & \multicolumn{2}{c|}{$\%$ F68 in solution } & F127 on & F68 on \\
\cline { 2 - 7 } & $\mathbf{0 . 0 8 \%}$ & $\mathbf{0 . 4 0 \%}$ & $\mathbf{0 . 8 0 \%}$ & $\mathbf{0 . 4 0 \%}$ & $\mathbf{0 . 8 0 \%}$ & surface & surface \\
\hline FITC- & 4.0 & 2.6 & 3 & 16.0 & 5.6 & 67.9 & 80.6 \\
Casein & $(0.7)$ & $(0.7)$ & $(0.4)$ & $(11.5)$ & $(1.9)$ & $(47.7)$ & $(17.2)$ \\
\hline & 0.7 & 0.5 & 0.5 & 0.6 & 0.3 & 49.3 & 67 \\
FITC-BSA & $(0.2)$ & $(0.1)$ & $(0.1)$ & $(0.2)$ & $(0.1)$ & $(9.1)$ & $(7.0)$ \\
\hline Alexa Fluor- & 0.3 & 0.6 & 0.9 & 0.2 & 0.5 & 155 & 148 \\
488 Fb & $(0.1)$ & $(0.3)$ & $(1.0)$ & $(0.1)$ & $(0.1)$ & $(20.7)$ & $(24.6)$ \\
\hline
\end{tabular}

${ }^{a} \mathrm{SD}$ in parentheses 
(a)

FITC-Casein Only

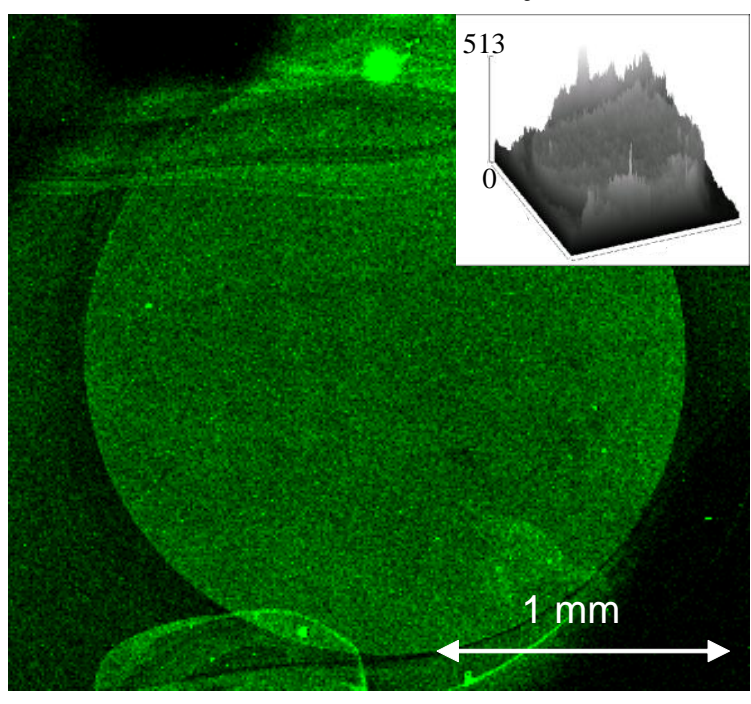

FITC-Casein + 0.08\% F127

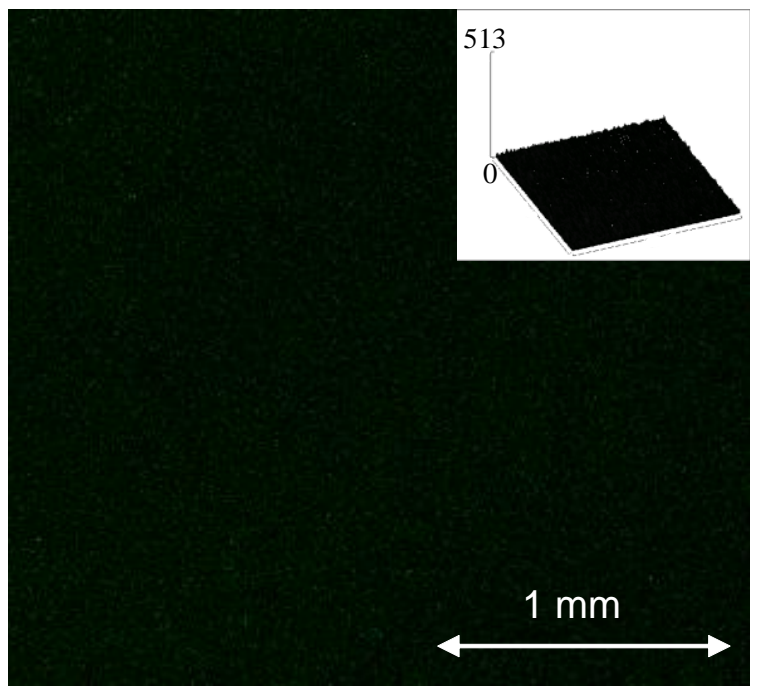

(b)

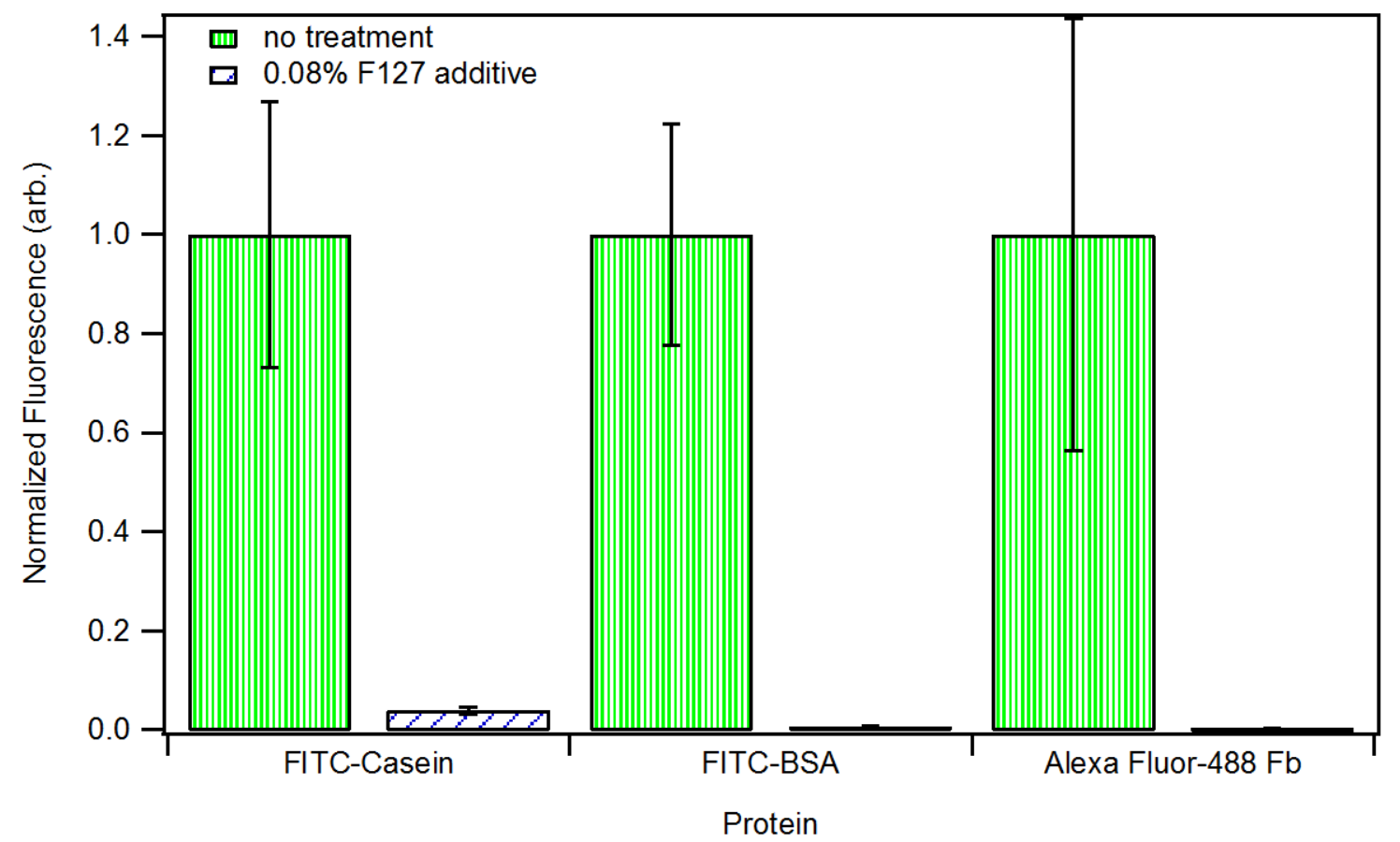

Figure 2.2: Confocal fluorescence analysis of the effect of Pluronic additive on protein adsorption to Tefloncoated surfaces. (a) Fluorescent images of test substrates exposed to FITC-casein without (left) and with (right) $0.08 \% \mathrm{w} / \mathrm{v}$ F127. The inset figures are 3D-graphs of pixel intensity. (b) Graph depicting the normalized, integrated fluorescence of substrates exposed to FTIC-casein, FITC-BSA and Alexa Fluor-488 Fb, without (diagonal-lined green bars) and with (vertical-lined blue bars) $0.08 \%$ Pluronic F127. The data represent six replicates per condition, with error bars of \pm 1 S.D. 
In comparison to strategy 1 for blocking protein adsorption (solution additives), strategy 2 (prepassivated surfaces) was not nearly as successful. In fact, as shown in Table 2.1, in the case of fibrinogen, prepassivation of surfaces appeared to increase the level of protein adsorption relative to the control. We are unsure of why this was the case because surface passivation has proven effective for other applications; ${ }^{87,94}$ we speculate that higher concentrations of Pluronics and longer passivation times may be required to block protein adsorption more effectively. Alternatively, covalent attachment of Pluronics or other PEO-containing polymers to surfaces $^{101,102}$ might be a more effective method. Regardless, because strategy 1 was so successful, we chose to adopt it, and in all of the experiments described in the following sections, $0.08 \% \mathrm{w} / \mathrm{v}$ F127 was used to prevent protein adsorption.

\subsubsection{Validation of Results}

To verify the confocal microscopy results described above (section 2.3.2), SIMS was used to qualitatively assess the effects of Pluronic additives on protein adsorption. In these experiments, unlabeled BSA was used as a model protein, and three experimental conditions were evaluated in which Teflon-coated surfaces were exposed to (i) $0.08 \% \mathrm{~F} 127$ alone, (ii) $1 \mathrm{mg} / \mathrm{mL}$ protein alone, and (iii) $1 \mathrm{mg} / \mathrm{mL}$ protein $+0.08 \%$ F127. As shown in Figure 2.3, several $\mathrm{m} / \mathrm{z}$, ratios were identified that correlated with particular chemical groups. For example, $m / z 50$ corresponds to Teflon (CF2') and appears in all samples, whereas $\mathrm{m} / \mathrm{z} 33,42$, and 26 (not shown) correspond to moieties found only in proteins $\left(\mathrm{SH}^{-}, \mathrm{CNO}^{-}\right.$, and $\mathrm{CN}^{-}$, respectively), and were detected only on surfaces exposed to protein alone (condition ii). The fact that these peaks were not detected in the sample containing protein and F127 (condition iii) supports the conclusion drawn from the confocal data that $0.08 \%$ F127 blocks the adsorption of proteins onto Teflon-coated surfaces.

In an attempt to interrogate the potential adhesion of Pluronic molecules to DMF substrates, we also evaluated several $\mathrm{m} / \mathrm{z}$ ratios that correspond to chemical groups in Pluronics $(\mathrm{m} / \mathrm{z}$ 59, 73, and 87) but were unable to detect any such peaks that were unique to conditions $\mathrm{i}$ and iii. Whereas F127 may adsorb to surfaces at levels below the detection limit of ToF-SIMS, it does not appear to do so to a significant extent. 


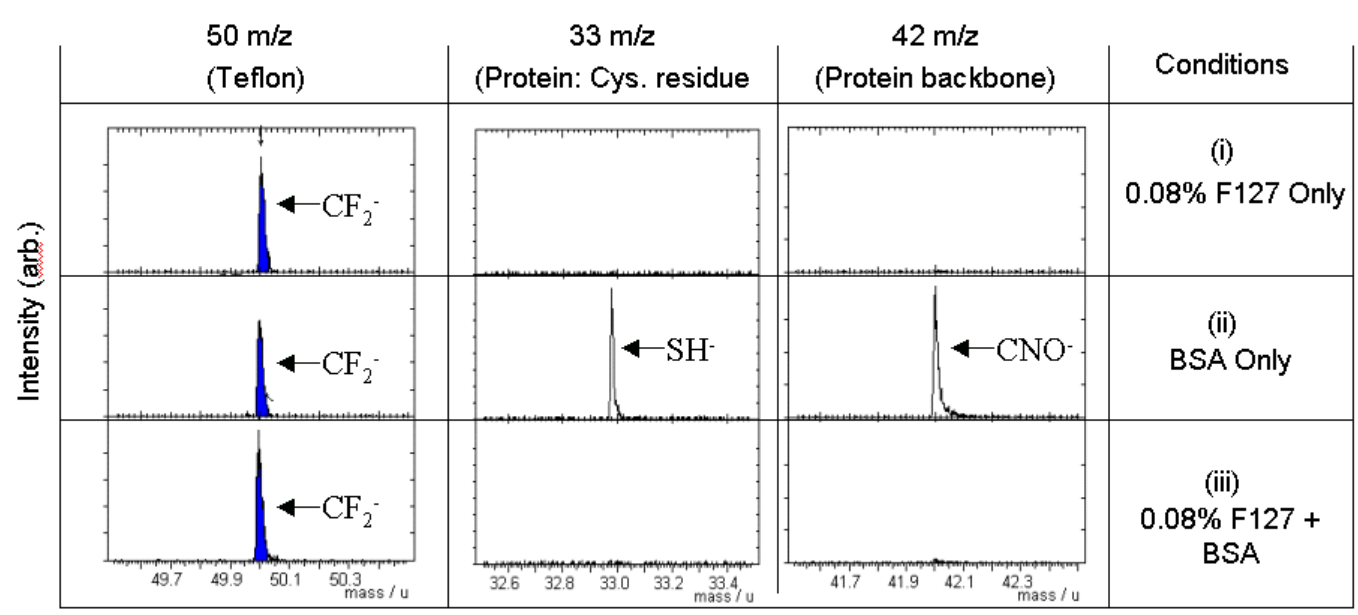

Figure 2.3. Negative ion SIMS spectra of Teflon-coated substrates exposed to (i) $0.08 \% \mathrm{~F} 127$ in Tris-HCl buffer; (ii) $1.0 \mathrm{mg} / \mathrm{mL}$ BSA in Tris-HCl buffer; and (iii) $1.0 \mathrm{mg} / \mathrm{mL}$ BSA in $0.08 \% \mathrm{~F} 127$ in Tris-HCl buffer. M/z 50, 33, and 42 correspond to $\mathrm{CF}_{2}^{-}, \mathrm{SH}^{-}$, and $\mathrm{CNO}^{-}$, respectively. The data shown are representative of 4 samples, evaluated with a scan area of $500 \times 500 \mu \mathrm{m}$.

\subsubsection{NMR investigation of Pluronic-Protein Interaction}

Pulsed Field Gradient NMR (PFG-NMR) is a well-established method used to study molecular diffusion in isotropic liquids, ${ }^{103-106}$ and can be applied to examine the diffusion of Pluronics in aqueous solutions. This method can be used to determine the presence of non-specific interactions between polymers and proteins as a function of the diffusant's (i.e., the polymer's) hydrodynamic radius. The natural logarithm of the normalized stimulated echo intensity decay, $I$, of F127 is shown as a function of $k$ in Figure 2.4. Here, $\mathrm{k}=\left(\gamma_{\mathrm{H}} \delta \mathrm{g}\right) 2(\Delta-\delta / 3)$, where $\gamma$ is the magnetogyric ratio of the nucleus under investigation (for ${ }^{1} \mathrm{H}, \gamma_{\mathrm{H}}=42.576 \mathrm{MHz} / \mathrm{T}$ ), $\delta$ is the gradient duration ( $5 \mathrm{~ms}$ ), $\mathrm{g}$ is the gradient amplitude (see above), and $\Delta$ is the diffusion time (400 ms). The slope of the plot is proportional to the diffusion coefficient of Pluronic F127. The diffusion coefficient of Pluronic F127 was determined in the presence and absence of BSA to be $5.22 \times 10^{-11} \mathrm{~m}^{2} \mathrm{~s}^{-1}$ and $5.58 \times 10^{-11} \mathrm{~m}^{2} \mathrm{~s}^{-1}$, respectively. Thus, the diffusion coefficient of F127 is relatively constant, regardless of the presence of BSA, which suggests that there is not much interaction between the two species. In this case, the difference between the diffusion coefficients with and without BSA is $6.7 \%$, which indicates the interaction between the two species is limited, and may simply be a result of the increase in the solution viscosity caused by the addition of proteins. 


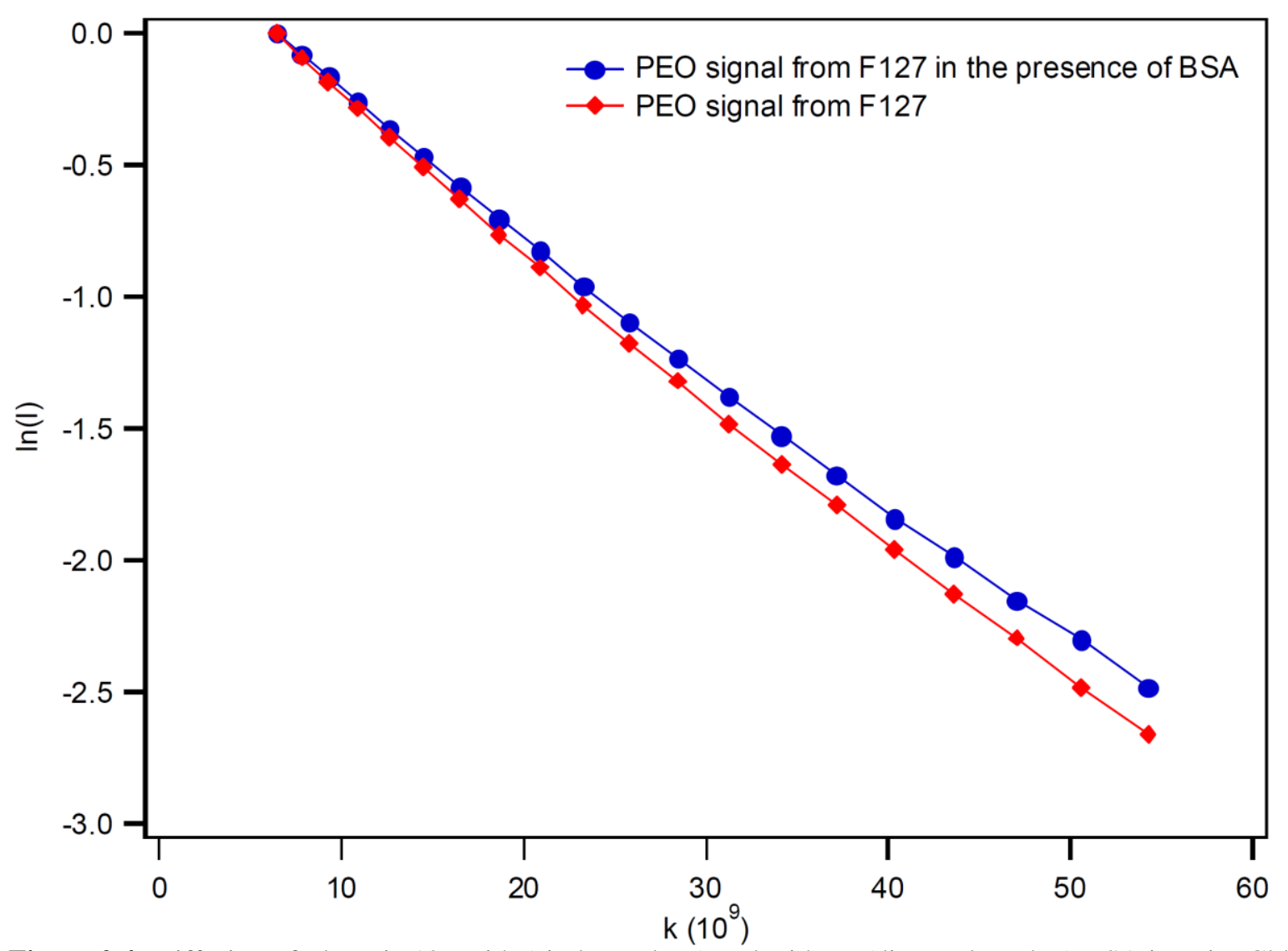

Figure 2.4: Diffusion of Pluronic 127 with (circle markers) and without (diamond marker) BSA in TrisHCl buffer. Plot of intensity decay $\left({ }^{1} \mathrm{H}\right.$ resonance of the PEO units in F127) as a function of $k$, where $\mathrm{k}=(\gamma \delta g)^{2}(\Delta-\delta / 3)$. Best fit line for free diffusion of F127 (red line) is $\mathrm{y}=-5.58 \times 10^{-11} x+0.30\left(\mathrm{R}^{2}=0.9984\right)$ and for diffusion of F127 in the presence of BSA (blue line) is $\mathrm{y}=-5.22 \times 10^{-11} x+0.29\left(\mathrm{R}^{2}=0.9983\right)$. The slope of the graph is proportional to diffusion coefficient: $\mathrm{D}_{\mathrm{F} 127}=-5.58 \times 10^{-11}$ and $\mathrm{D}_{\mathrm{F} 127 \text { (with } \mathrm{BSA})}=-5.22 \times 10^{-11}$.

\subsubsection{Effects and Mechanism of Pluronic Additives}

The additive strategy for preventing protein adsorption in digital microfluidics has potential drawbacks because chemical additives can interfere with sensitive assay constituents such as enzymes or cells. Fortunately, this does not appear to be a problem for pluronics. For example, PEG-containing polymers have been shown not to cause problems for protein-protein interactions ${ }^{107}$ and in fact can be used as biomimetic additives to enhance protein stability. ${ }^{108,109}$ For this reason, Pluronics are widely used in tissue engineering studies ${ }^{110,111}$ and is even included in some formulations of commercial cell culture media. ${ }^{112}$ These data are supported by our own work, which has demonstrated little or no effect of Pluronic additives on cell viability ${ }^{71}$ or 
enzyme kinetics. ${ }^{72}$ Finally, we note that the concentration reported here, $0.08 \% \mathrm{w} / \mathrm{v}$ F127, is probably not the lower-limit on conditions that can reduce biofouling; it is simply the lowest concentration we evaluated. Thus, if $0.08 \%$ F127 ever proves problematic for a given assay, lower concentrations might be effective.

The data presented in Figures 2.2 and 2.3 do not suggest an obvious mechanism: how does a small amount of additive so effectively reduce the level of protein adsorption onto Teflon-coated substrates? The molar ratios of $0.08 \%$ Pluronics/protein in these experiments were 1:6.9, 1:2.4, and 1:0.5 for casein, $\mathrm{BSA}$, and $\mathrm{Fb}$, respectively; thus, it is conceivable that the observed effect on adsorption is a function of interactions between additive and protein molecules (e.g., it is possible that Pluronic molecules coat the proteins, preventing them from adsorbing to surfaces). In experiments described in the section 2.3.4, we probed this phenomenon using stimulated echo (STE) pulsed field gradient (PFG) NMR to calculate diffusion coefficients for Pluronic F127. As shown in Figure 2.4, the presence of BSA has little effect on the diffusion coefficient of the additive, suggesting that molecular interactions (if any) are limited. More study is warranted, but it seems unlikely that the effects reported above can be explained by additive-protein interactions.

We hypothesize that the observed effects on protein adsorption are a result of layers of Pluronics that form at the phase boundaries of aqueous droplets. We base this hypothesis on the results of Chen et al., ${ }^{113}$ who used vibrational spectroscopy to demonstrate that Pluronics dissolved in water at concentrations between $0.0001 \%$ and $0.5 \% \mathrm{w} / \mathrm{v}$ form an ordered layer at the air/liquid and hydrophobic solid/liquid interfaces (PPO units oriented toward air or solid, PEO oriented toward water). In similar studies, Phipps et al. ${ }^{114}$ used neutron reflection to probe the same phenomenon, revealing that ordered layers of Pluronics form at interfaces when dissolved at concentrations $>0.001 \% \mathrm{w} / \mathrm{v}$. If Pluronic layers form at the solid/liquid interface in droplets manipulated by digital microfluidics (even if the layers are not permanent, as indicated by the fact that we did not detect Pluronics-derived signals by SIMS), this could prevent protein adsorption, even if proteins are present at much higher concentrations than Pluronics in the bulk solution (as is the case in the following section). 


\subsubsection{Non-Stick Digital Microfluidics}

The experiments described above demonstrate that Pluronic additives significantly reduce the extent of biofouling on Teflon surfaces. To test the efficacy of this strategy for digital microfluidics, we evaluated the maximum movable concentrations of three unlabeled proteins (casein, BSA, and Fb) with and without $0.08 \%$ F127. Movability was defined as the capacity to manipulate droplets back and forth over four electrodes on three different devices. As shown in Table 2.2, in control solutions without Pluronic additive, the maximum movable concentrations were $\sim 5-15 \mu \mathrm{g} / \mathrm{mL}$ (similar to what has been reported previously ${ }^{66-68}$ ). In comparison, when droplets contained $0.08 \%$ F127, the maximum movable concentrations were more than $1000 \times$ higher (i.e., 10-50 mg/mL). In fact, the concentrations reported in Table 2.2 for Pluronic containing samples are not limits; they are simply the highest concentrations we tested (and are likely much higher concentrations than are needed for most assays).

Table 2.2. Maximum Movable ${ }^{a}$ Protein Concentrations by DMF ( $\left.\mathrm{n}=3\right)$

\begin{tabular}{|c|c|c|c|c|}
\hline & Casein & BSA & Fb & 100\% FBS \\
\hline \multirow{2}{*}{ Protein Only } & $0.005 \mathrm{mg} / \mathrm{mL}$ & $0.005 \mathrm{mg} / \mathrm{mL}$ & $0.0015 \mathrm{mg} / \mathrm{mL}$ & Not movable \\
& & & & \\
\hline Protein + & At least & At least & At least & Movable \\
$\mathbf{0 . 0 8 \% ~ F 1 2 7 ~}$ & $10 \mathrm{mg} / \mathrm{mL}$ & $50 \mathrm{mg} / \mathrm{mL}$ & $50 \mathrm{mg} / \mathrm{mL}$ & \\
& $(1: 69)^{\boldsymbol{b}}$ & $(1: 120)^{\boldsymbol{b}}$ & $(1: 25)^{\boldsymbol{b}}$ & \\
\hline
\end{tabular}

${ }^{a}$ Definition of movability: droplet moves across four electrodes back and forth for two cycles on three different devices. ${ }^{b}$ Molar ratios of Pluronic/protein.

In an attempt to probe the limits of this phenomenon, we evaluated the movability of fetal bovine serum (FBS), a complex, concentrated mixture of proteins and other constituents. Somewhat surprisingly, this very sticky solution, when paired with $0.08 \%$ F127, was movable by DMF. The translation of FBS-containing droplets was sluggish relative to that of less concentrated solutions (probably caused by viscosity), but the results were reproducible and no signs of adsorption (i.e., irregularities in the circular droplet shape) were observed. These compelling results suggest that 
Pluronic additives transform DMF into a technique compatible with a much wider range of reagents, solutions, and assays than was previously thought to be possible.

To illustrate the new kinds of applications that are possible for digital microfluidics when paired with Pluronic additives, we implemented a DMF-driven protein digest assay. The reporter in this assay, bodipy-labeled/quenched casein, has low fluorescence when intact but becomes highly fluorescent when digested, making it useful for evaluating the kinetics of proteolysis. In this assay, a concentrated $(1 \mathrm{mg} / \mathrm{mL})$ solution of bodipy-casein was used to ensure that the fluorescence would be visible in a stereomicroscope. Note that this would be impossible without Pluronics because $1 \mathrm{mg} / \mathrm{mL}$ is more than $100 \times$ higher than the maximum movable concentration without the additive. Figure 2.5a shows a sequence of images from a movie depicting the assay: $70 \mathrm{~nL}$ droplets containing trypsin and bodipy-casein were dispensed from reservoirs and then merged, mixed, and allowed to react. As shown in frame 6 (Figure 2.5a), the droplet becomes fluorescent after $\sim 10 \mathrm{~min}$. In addition to visualization, the reaction was implemented (at lower concentrations) with quantitative detection using a fluorescence plate reader. As shown in Figure $2.5 \mathrm{~b}$, the progression of the reaction was monitored for $28 \mathrm{~min}$. Throughout the reaction, droplets were observed to be movable, suggesting that the combination of Pluronic additives with DMF will facilitate procedures composed of sequential reactions that require extended periods of time.

\subsection{Conclusion}

We have demonstrated that confocal microscopy is a useful tool for probing the extent of biofouling that occurs on digital microfluidic devices. Using this tool, we identified a simple strategy for limiting protein adsorption: the use of low concentrations of Pluronic additives. Secondary ion mass spectrometry was used to corroborate these results. On the basis of previous studies, we hypothesize that this effect is a result of the formation of ordered layers of Pluronics at the phase boundaries of aqueous droplets. Regardless of the mechanism, the additive strategy significantly limits the amount of biofouling such that 1000-fold higher concentrations are movable when compared to DMF without the additive. These compelling results suggest that Pluronic additives transform DMF into a technique compatible with a much wider range of reagents, solutions, and assays than was previously thought to be possible. 
(a)
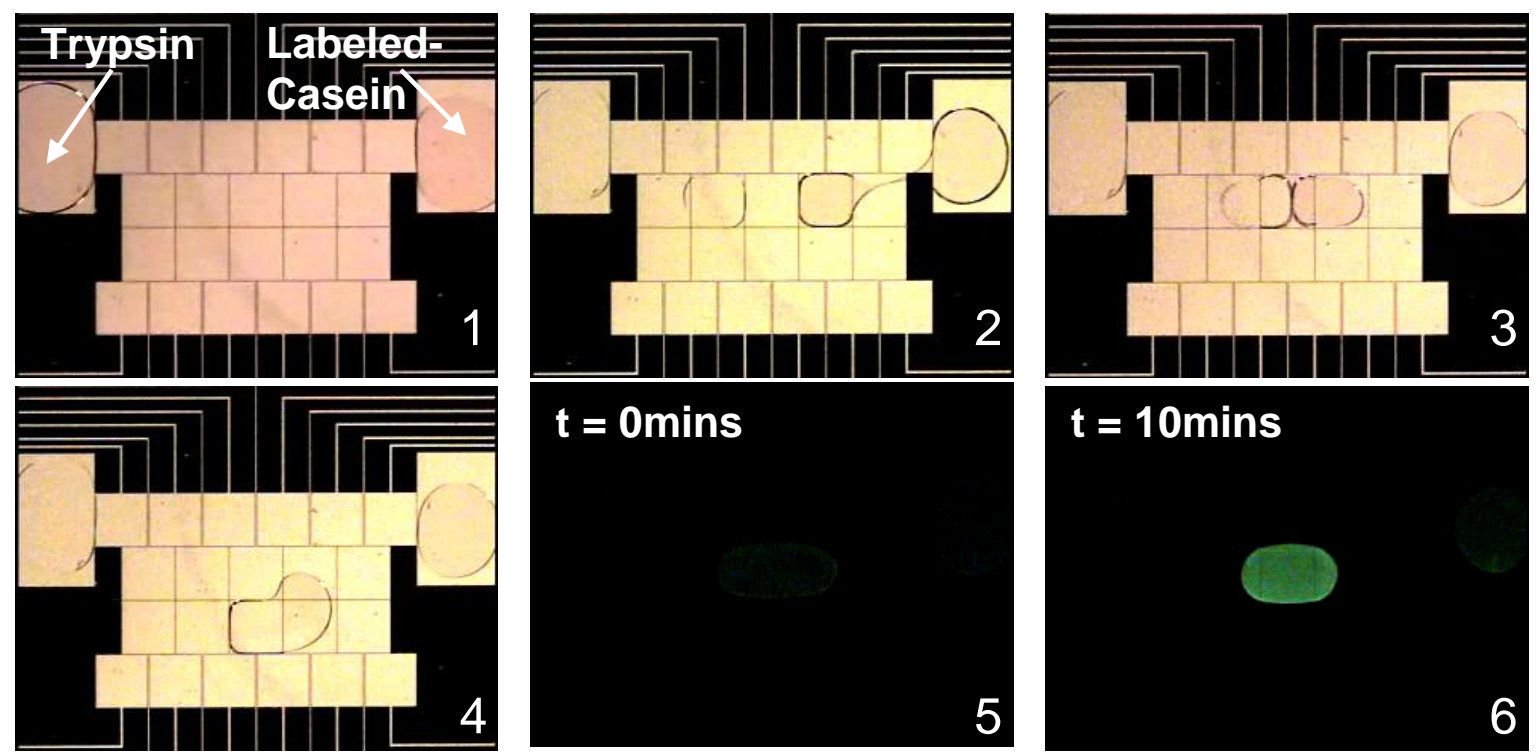

(b)

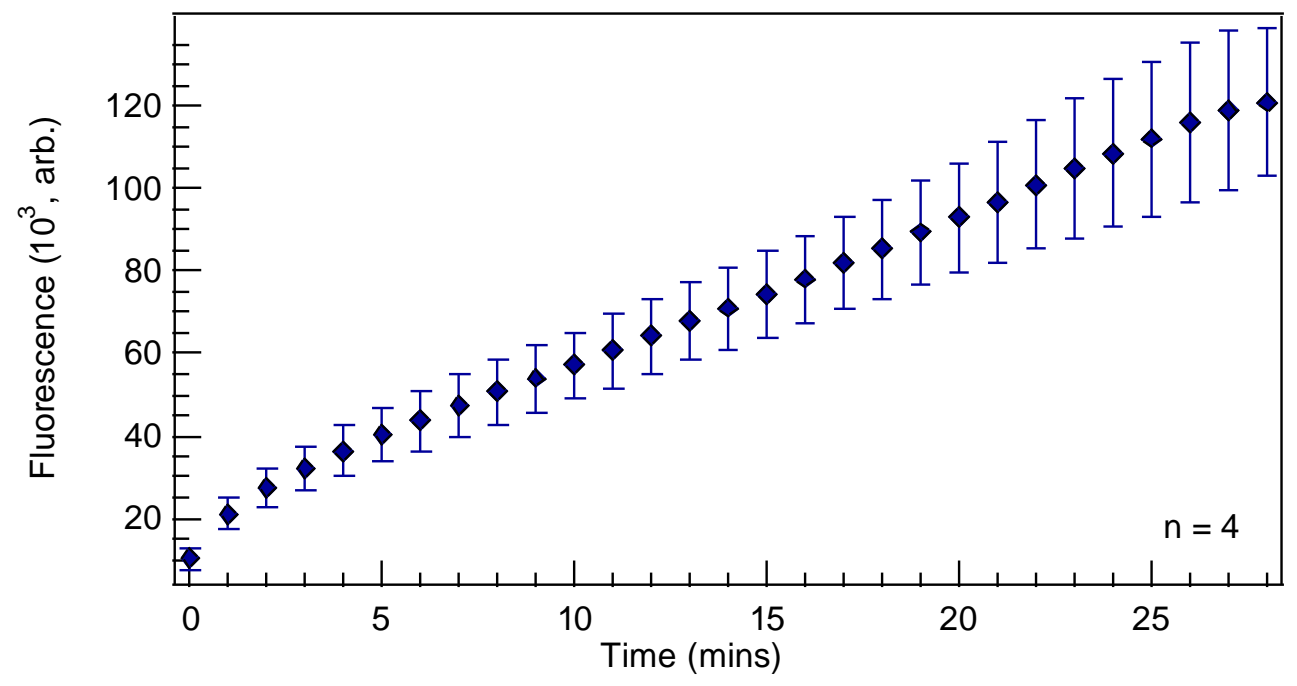

Figure 2.5: Digital microfluidic driven proteolysis assay. (a) Sequence of images depicting fluorescent assay for tryptic digestion. (1-2) Droplets containing Bodipy-casein $(1 \mathrm{mg} / \mathrm{mL})$ and trypsin $(100 \mu \mathrm{g} / \mathrm{mL})$ are dispensed from reservoirs, (3) merged and (4) actively mixed. (5-6) Fluorescent images of merged droplet before and after digestion. Fluorophores on undigested casein are quenched (i.e., non-fluorescent); as the protein is cleaved, the fluorescent signal increases. (b) Graph depicting the kinetics of tryptic digestion on a DMF device, as measured in a fluorescence plate reader. Error bars are \pm 1 S.D. and error in the data points ranged from $6-13 \%$ RSD. 


\section{Chapter 3 - A Digital Microfluidic Approach to Proteomic Sample Processing}

A common characteristic for proteomic analyses is the need for extensive biochemical processing. Digital microfluidics (DMF), a technique characterized by the manipulation of discrete microdroplets $(100 \mathrm{~nL}-10 \mu \mathrm{L})$ on an open array of electrodes, is a good match for carrying out rapid, automated solution-phase reactions. Here, we report a DMF-based method integrating several common processing steps in proteomics, including reduction, alkylation, and enzymatic digestion. Fluorogenic assays were used to quantitatively evaluate the kinetics and reproducibility of each reaction step, and MALDI-MS was used for qualitative confirmation. The method is fast, facile, and reproducible, and thus has the potential to be a useful new tool in proteomics.

\subsection{Introduction}

There is growing consensus that current proteomic analysis methods are limited by nonstandardized sample handling and processing. For example, the Human Proteome Organization (HuPO) recently published a comprehensive study of "preanalytical variables that can potentially impact the outcome of results, but are not related to inherent sample differences." "115 According to the study, a key source of variance is the ad hoc nature of sample processing methods used by laboratories around the world. In fact, the error introduced to analyses by non-standard processing is so great that different laboratories analyzing the same sample can obtain orthogonal results. It seems likely that the promise of proteomics for clinical applications ${ }^{116}$ will not be realized until these problems can be solved.

A technology that may provide some relief for ad hoc and irreproducible proteomic sample processing is miniaturization and integration in microfluidics. ${ }^{117}$ For example, networks of enclosed microchannels have been used to implement the key proteomic processing step of enzymatic digestion in a variety of formats, including trypsin-coated beads ${ }^{37,38}$ or membranes, ${ }^{118}$ and immobilized enzymes in sol-gels ${ }^{40,41}$ or polymer monoliths. ${ }^{119}$ These formats, which have high surface-area-to-volume ratios, have favorable reaction kinetics and are a good match for applications requiring a single processing step. However, we posit that enclosed channels are illsuited for carrying out multistep processing regimens. Diffusion and uncontrolled fluid 
flows ${ }^{120,121}$ make it difficult to control a complicated series of reactions between well-defined plugs of reagents in microchannels. Unfortunately, this challenge is typical in proteomics; for example, in shotgun proteomics, samples are subjected to a multiday, multistep procedure including acidification, denaturing, reduction, alkylation, enzymatic digestion (twice), purification, and dilution prior to analysis by separations and mass spectrometry. ${ }^{13}$ We acknowledge that microvalves ${ }^{48}$ may offer some relief from this problem, but we submit that in general, microchannels are ill-suited for implementing the complicated series of reactions necessary for many proteomic applications (with some exceptions noted ${ }^{47}$ ). In contrast to microchannels, we propose that digital microfluidics (DMF) is a good match for implementing multistep series of reactions. $\mathrm{DMF}^{122,123}$ is a fairly new fluid handling technique characterized by the manipulation of discrete droplets on an open array of electrodes. ${ }^{53,54}$ By applying a sequence of potentials to adjacent electrodes, a droplet of fluid can be dispensed from a reservoir, transported on the array, and merged with other droplets to implement nanoliter-scale reactions. Because each droplet is isolated from its surroundings rather than being embedded in a stream of fluid, DMF is a facile method for forming microreactors in which there is no possibility that reagents or samples will diffuse away. Most importantly, because each droplet is controlled individually, multistep methods involving many different reagents can be easily programmed.

In the past, digital microfluidics has primarily been used for relatively simple applications comprising one or two steps, including cell-based assays, ${ }^{71}$ enzyme assays, ${ }^{72,75}$ sample preparation for mass spectrometry, ${ }^{66-68}$ immunoassays, ${ }^{124,125}$ and the polymerase chain reaction. ${ }^{77}$ Here, we report an integrated method for implementing three discrete processing steps, including proteomic sample reduction, alkylation, and digestion; this paper joins related work ${ }^{52,126,127}$ in demonstrating the compatibility of DMF with multistep proteomic sample processing. The qualitative and quantitative data presented here suggest that DMF is capable of highly repeatable sample processing, and thus may be a useful new tool for standardized proteomics analyses.

\subsection{Materials and Method}

\subsubsection{Reagents and Materials}

Bovine serum albumin (BSA), lysozyme from chicken egg white, insulin from bovine pancreas, TPCK-treated trypsin from bovine pancreas, pepsin from porcine gastric mucosa, endoproteinase Lys-C (Lys-C), iodoacetamide, Tris-HCl, Pluronic F127, Fluorinert FC-40, acetonitrile (ACN), 
and acetic acid were purchased from Sigma Chemical (Oakville, ON).Tris-(2carboxyethyl)phosphine hydrochloride (TCEP) and E6638 EnzChek Protease Assay Kit were purchased from Invitrogen (Burlington, ON). Ziptip pipet tips $\left(\mathrm{C}_{4}\right.$ and $\left.\mathrm{C}_{18}\right)$ were purchased from Millipore (Etobicoke, ON), and R-cyano-4-hydroxycinnamic acid (R-CHCA) and sinapinic acid (SA) were purchased from Waters Limited (Mississauga, ON). Unless otherwise indicated, all protein and processing reagent solutions were prepared in working buffer (100 mM Tris- $\mathrm{HCl}, \mathrm{pH}$ 7.8, $0.08 \%$ w/v Pluronic F127) immediately prior to use. Clean room reagents and supplies included Shipley S1811 photoresist and MF321 developer from Rohm and Haas (Marlborough, MA), AZ300T photoresist stripper from AZ Electronic Materials (Somerville, NJ), solid chromium from Kurt J. Lesker Canada (Toronto, ON), CR-4 chromium etchant from Cyantek (Fremont, CA), hexamethyldisilazane (HMDS) from Shin-Etsu MicroSi (Phoenix, AZ), and conc. sulfuric acid and hydrogen peroxide (30\%) from Fisher Scientific Canada (Ottawa, ON). Piranha solution was prepared as a 3:1 (v/v) mixture of sulfuric acid and hydrogen peroxide. Parylene-C dimer was from Specialty Coating Systems (Indianapolis, IN), and Teflon-AF was from DuPont (Wilmington, DE).

\subsubsection{Device Fabrication}

Digital microfluidic devices were fabricated in the University of Toronto Emerging Communications Technology Institute (ECTI) facility using chrome-on-quartz photomasks printed at the University of Alberta NanoFab (Edmonton, AB). Briefly, glass wafers were cleaned in piranha solution (10 $\mathrm{min})$, and then coated with chromium (200 $\mathrm{nm}$ ) by electron beam deposition. After rinsing and baking on a hot plate $\left(115^{\circ} \mathrm{C}, 5 \mathrm{~min}\right)$, the substrates were primed by spin-coating with HMDS (3000 rpm, 30 s) and were then spin-coated with Shipley S1811 photoresist $(3000 \mathrm{rpm}, 30 \mathrm{~s})$. Substrates were pre-baked on a hot plate $\left(100^{\circ} \mathrm{C}, 2 \mathrm{~min}\right)$ and exposed through a photomask using a Suss Mikrotek mask aligner. Substrates were developed in MF321 (3 min), and then post-baked on a hot plate $\left(100^{\circ} \mathrm{C}, 1 \mathrm{~min}\right)$. After photolithography, substrates were immersed in chromium etchant (30 s). Finally, the remaining photoresist was stripped in AZ300T (10 min).

After forming electrodes, devices were coated with parylene-C (2 $\mu \mathrm{m})$ and Teflon-AF (50 nm). Parylene-C was applied using a vapor deposition instrument (Specialty Coating Systems), and Teflon-AF was spin-coated (1\% w/w in Fluorinert FC-40, 2000 rpm, 60 s) and then post-baked 
on a hot-plate $\left(160^{\circ} \mathrm{C}, 10 \mathrm{~min}\right)$. To facilitate the application of driving potentials, the polymer coatings were locally removed from the contact pads by gentle scraping with a scalpel. In addition to patterned devices, unpatterned indium tin oxide (ITO) coated glass substrates (Delta Technologies Ltd., Stillwater, MN) were coated with Teflon-AF (50 nm, as above).

\subsubsection{Device Operation}

Devices were assembled with an unpatterned ITO/glass top plate and a patterned bottom plate separated by a spacer formed from one piece of double-sided tape ( $\sim 70 \mu \mathrm{m}$ thick). Droplets were sandwiched between the two plates and actuated by applying electric potentials between the top electrode and sequential electrodes on the bottom plate. The driving potentials of $60-80 \mathrm{~V}_{\text {rms }}$ were generated by amplifying the output of a function generator operating at $18 \mathrm{kHz}$, and were applied manually to exposed contact pads on the bottom plate surface. Droplet actuation was monitored and recorded by a CCD camera mounted on a stereomicroscope with fluorescence imaging capability (Olympus Canada, Markham, ON). The devices used here had four $2.5 \times 2.5$ $\mathrm{mm}$ reservoir electrodes mated to a $4 \times 6$ array of $1 \times 1 \mathrm{~mm}$ actuation electrodes, with interelectrode gaps of $5-40 \mu \mathrm{m}$. Each "unit" droplet covered a slightly larger area than a single actuation electrode such that the volume in each droplet was $\sim 100 \mathrm{~nL}$.

\subsubsection{Qualitative Analysis}

The proteomic processing work-flow was analyzed in four stages: (i) prior to reaction, (ii) after reduction, (iii) after alkylation, and (iv) after digestion. Insulin (40 $\mu \mathrm{M}$ in working buffer) was used as a model analyte for evaluating stages (i-iii). For stage (i), a droplet of insulin was dispensed by DMF and then analyzed. For stage (ii), droplets of insulin and TCEP (10 mM in working buffer) were dispensed and merged by DMF followed by incubation (room temp., 30 min). For stage (iii), droplets of insulin and TCEP were dispensed, merged and incubated (as in stage ii), and the product was then reacted with a third droplet of iodoacetamide (12 $\mathrm{mM}$ in working buffer) and incubated again (room temp, $2 \mathrm{~min}$ ). Lysozyme (40 $\mu \mathrm{M}$ in working buffer) and BSA (10 $\mu \mathrm{M}$ in working buffer) were used as model analytes for stage (iv). In these experiments, droplets of lysozyme or BSA were dispensed and processed as in stage (iii) (above), followed by reaction with a droplet of trypsin (1:5 w/w of enzyme:protein in working buffer) and incubation (room temp, $60 \mathrm{~min}$ ). In all cases, incubation steps were carried out in a 
humidified chamber (a Petri dish partially filled with deionized water, $\mathrm{dH}_{2} \mathrm{O}$ ) to limit evaporation. At least three replicate samples were evaluated for each condition.

After processing by DMF, each sample was purified using a C18 ZipTip and then analyzed by matrix assisted laser desorption/ ionization mass spectrometry (MALDI-MS). Briefly, ZipTips were wetted in $100 \% \mathrm{ACN}$ containing $0.1 \%$ acetic acid $(3 \times)$ and then equilibrated in $10 \% \mathrm{ACN}$ containing $0.1 \%$ acetic acid $(3 \times)$. Aqueous samples were drawn in and out of tips for 7-10 cycles, and then the solid medium was washed with $10 \%$ ACN containing $0.1 \%$ acetic acid $(3 \times)$. Finally, samples were eluted in $100 \%$ ACN containing $0.1 \%$ acetic acid $(2 \times 1 \mu \mathrm{L})$ onto a stainless steel MALDI target plate. A $1 \mu \mathrm{L}$ matrix solution was added to each sample: SA (10 $\mathrm{mg} / \mathrm{mL}$ in 50:50 ACN: $\mathrm{dH}_{2} \mathrm{O}$ containing 0.1\% acetic acid) for intact proteins (stage $\mathrm{i}$ ), and $\alpha$ CHCA $\left(10 \mathrm{mg} / \mathrm{mL}\right.$ in 50:50 ACN: $\mathrm{dH}_{2} \mathrm{O}$ containing 0.1\% acetic acid) for reduced, alkylated, and digested products (stages ii-iv). After drying, spots were analyzed using a Micro-MX mass spectrometer (Waters, Milford, MA) operating in positive and reflectron modes over a mass to charge ratio $(\mathrm{m} / \mathrm{z})$ range from $500-6,500$. At least one hundred shots were collected per spectrum, with laser power tuned to optimize the signal-to-noise ratio $(\mathrm{S} / \mathrm{N})$. Data were

processed by normalization to the largest analyte peak, baseline subtraction, and smoothed with a 15-point running average. Spectra of enzyme digests were analyzed using the Mascot protein identification package searching the SwissProt database, with 3 allowed missed cleavages, a mass accuracy of $\pm 1.2 \mathrm{Da}$, and with a fixed carbamidomethyl modification.

\subsubsection{Reaction Rate Analysis}

Fluorogenic assays were used to evaluate reaction rates of reduction and digestion on DMF devices. For the former, the analytes were BSA (10 $\mu \mathrm{M}$ in working buffer), insulin (40 $\mu \mathrm{M}$ in working buffer), and lysozyme (40 $\mu \mathrm{M}$ in working buffer), the reductant was TCEP (10 $\mathrm{mM}$ in working buffer), and the reporter was ABD-F (12 $\mathrm{mM}$ in working buffer). In each reaction, three droplets containing analyte, TCEP, and ABD-F were dispensed, merged, and rapidly mixed. Reaction progress was followed by fluorescence detection with $\lambda_{\mathrm{ex}}=390 \mathrm{~nm}$ and $\lambda_{\mathrm{em}}=$ $510 \mathrm{~nm}$. For the latter (digestion assays), the proteases were Lys-C $(0.07 \mathrm{mg} / \mathrm{mL}$ in working buffer $)$, pepsin $(0.07 \mathrm{mg} / \mathrm{mL}$ in $100 \mathrm{mM} \mathrm{HCl}, \mathrm{pH} 2.0$ containing 0.08\% w/v Pluronic F127), and trypsin $(0.07 \mathrm{mg} / \mathrm{mL}$ in working buffer $)$, and the reporter/analyte was the EnzChek reagent, intramolecularly quenched, fluorescently labeled BODIPY-Casein $(0.33 \mathrm{mg} / \mathrm{mL}$ in working 
buffer). In each case, droplets of BODIPY-Casein and enzyme were dispensed, merged, and rapidly mixed, and then evaluated by fluorescence detection with $\lambda_{\mathrm{ex}}=485 \mathrm{~nm}$ and $\lambda_{\mathrm{em}}=520 \mathrm{~nm}$.

In both types of assays, the fluorescence was quantified using a PheraStar multiwell plate reader (BMG Labtech, Durham, NC) as described previously (chapter 2). ${ }^{50,71,72,128}$ In each assay, fluorescence from processed samples was measured in intervals of 10 min for $1 \mathrm{~h}$. Between intervals, devices containing the reacting droplets were stored in a humidified chamber to prevent evaporation. Three replicate trials were conducted for each condition, and data were normalized and fitted with exponential functions.

\subsubsection{Reproducibility Assay}

The reproducibility of the reduction and digestion assays was evaluated using methods similar to those described above. Briefly, for reduction, droplets containing 6 picomole aliquots of BSA, insulin, or lysozyme ( $\sim 100 \mathrm{~nL}$ of $60 \mu \mathrm{M}$ protein in working buffer) were dispensed and mixed with $\sim 100 \mathrm{~nL}$ droplets of TCEP (10 mM in working buffer) and ABD-F (12 mM in working buffer). For digestion, droplets containing $0.17 \mu \mathrm{g}$ aliquots of BODIPY-Casein $(\sim 100 \mathrm{~nL}$ of 1.7 $\mathrm{mg} / \mathrm{mL}$ protein in working buffer) were dispensed and mixed with $\sim 100 \mathrm{~nL}$ droplets of one of either Lys-C $(0.3 \mathrm{mg} / \mathrm{mL}$ in working buffer $)$, pepsin $(0.3 \mathrm{mg} / \mathrm{mL}$ in $100 \mathrm{mM} \mathrm{HCl}, \mathrm{pH} 2.0$ containing $0.08 \% \mathrm{w} / \mathrm{v}$ Pluronic F127), or trypsin $(0.3 \mathrm{mg} / \mathrm{mL}$ in working buffer). For both assays, the final combined droplets were allowed to incubate (45 min) in a humidified chamber and were then evaluated using a PheraStar multiwell plate

\subsection{Results and Discussion}

\subsubsection{Multistep Proteomic Processing on DMF Devices}

The DMF array geometry is a natural fit for carrying out sequential biochemical assays, such as proteomic sample processing. The device shown in Figure 3.1 was designed for handling proteins and proteomic reagents to facilitate three sequential reactions: reduction of disulfides, alkylation of free thiols, and proteolytic digestion. As shown, in a typical analysis, a droplet containing the sample to be processed (in this case, insulin), was dispensed (Figure 3.1b) and merged with a droplet containing reducing agent (TCEP). The stability, selectivity, and rapid reaction rate at room temperature makes TCEP a good replacement for the more commonly used dithiothreitol (DTT), which must be removed prior to further processing. ${ }^{129}$ After reduction 
(Figure 3.1c), the sample droplet was merged with a droplet containing iodoacetamide (Figure 3.1d), which covalently binds to cysteine groups preventing reformation of disulfide bonds. Finally, the sample droplet was merged with a droplet containing protease (in this case, trypsin) to digest the protein into constitutive peptides (Figure 3.1e).

MALDI-MS was used to qualitatively evaluate DMF-driven processing reactions; Figure 3.2 shows representative spectra collected from each step in the process. As expected, the most significant feature in spectra of non-processed insulin (Figure 3.2a) is a peak at $5.7 \mathrm{kDa}$, corresponding to the singly charged intact analyte, while the smaller peak at $2.9 \mathrm{kDa}$ is its doubly charged equivalent. Insulin is made up of two subunits, an $\alpha$-chain $(2.3 \mathrm{kDa})$ and $\beta$-chain $(3.4$ $\mathrm{kDa}$ ), that are held together by disulfide bonds, ${ }^{130}$ and peaks corresponding to each of them are apparent in spectra collected after reduction (Figure 3.2b). After reduction, reaction with iodoacetamide adds $57 \mathrm{Da}$ to each thiol group; as shown in Figure 3.2c, after intermediate reaction times, several peaks are observed corresponding to varying degrees of alkylation (where $v$ represents the number of reacted thiol groups). When reacted for longer times $(\sim 10 \mathrm{~min})$, the analytes were completely alkylated (spectra not shown), but even after intermediate times, the peak representing the unreacted sample at $5.7 \mathrm{kDa}$ has essentially disappeared. Spectra of insulin samples after digestion (not shown) confirm the efficacy of the complete regimen. We also applied on-chip reduction, alkylation, and digestion to larger analytes such as BSA (Figure 3.2d) and lysozyme (Figure 3.2e). Asterisks in these spectra denote peptides identified by the database search engine, Mascot. These data demonstrate that DMF is a robust tool capable of implementing all of the key steps in proteomic processing. 

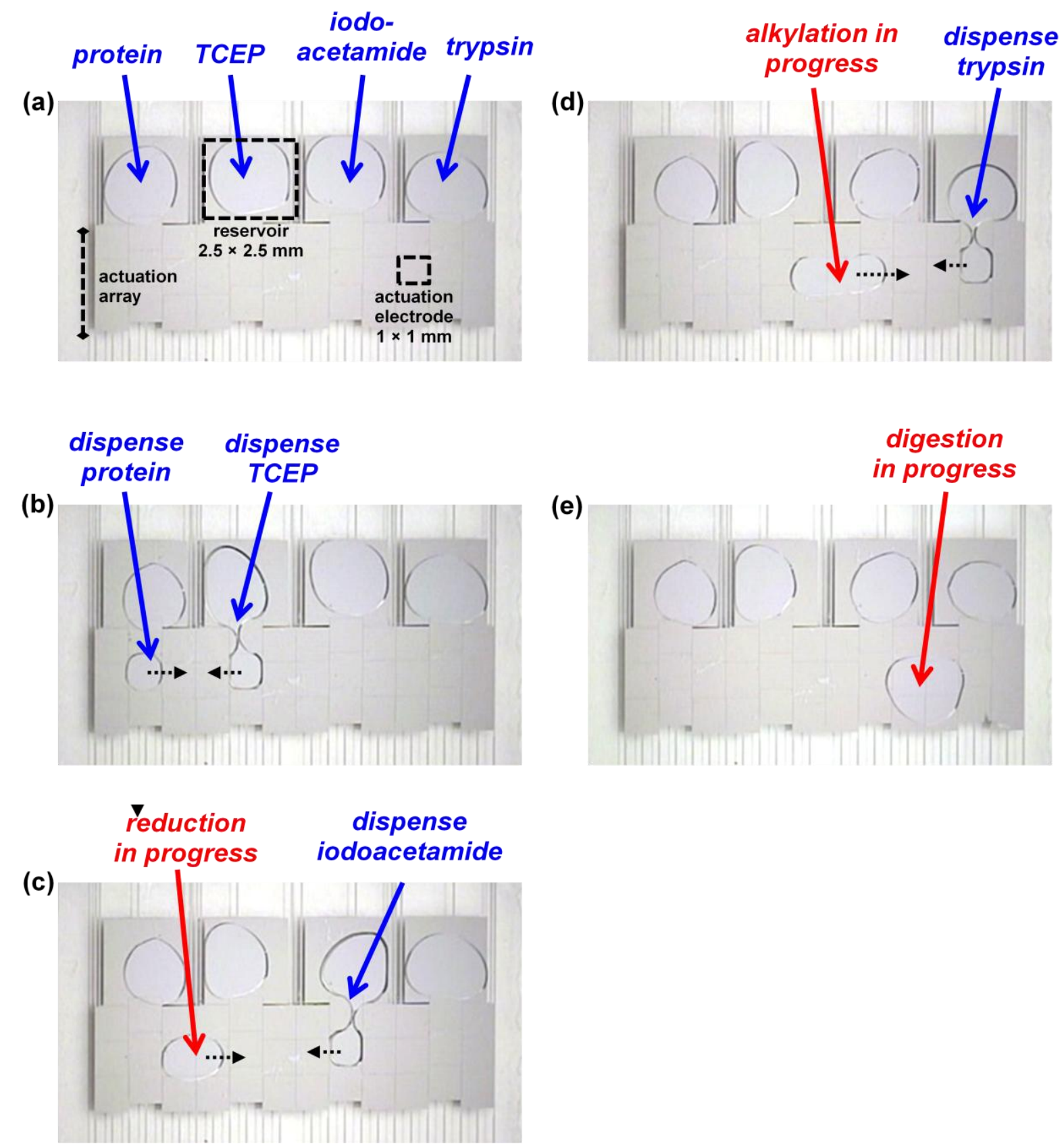

Figure 3.1: Sequence of images depicting a typical proteomic sample processing workup. (a-b) Droplets containing insulin $(40 \mu \mathrm{M})$ and TCEP $(10 \mathrm{mM})$ are dispensed from reservoirs, merged and actively mixed. After incubating, (c) a droplet of iodoacetamide $(12 \mathrm{mM})$ is dispensed and merged with the sample droplet and actively mixed. After incubating again, (d) the sample droplet is merged with a droplet of trypsin (1:5 enzyme to protein ratio w/w) and then (e) incubated a final time. 

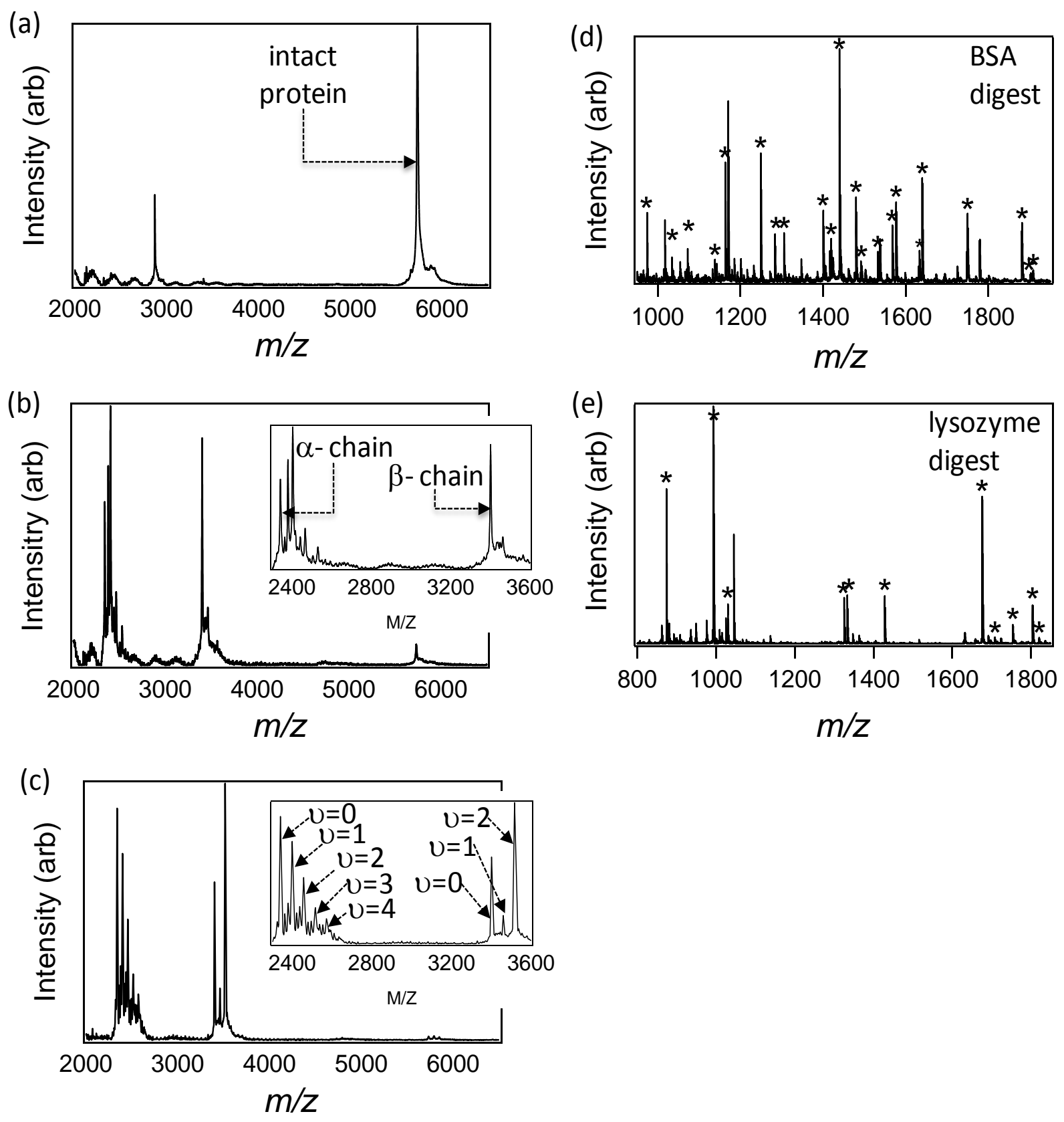

Figure 3.2: Representative MALDI-MS spectra of samples prepared by DMF-driven processing. (a) Nonprocessed insulin, (b) insulin after reduction (c) insulin after reduction and alkylation, where $v$ denotes the number of alkylated thiols and (d, e) BSA and lysozyme after tryptic digestion. In the latter, asterisks denote peptides identified by Mascot; Mowse scores were 167 for both spectra, yielding correct sample identifications $(p<0.05)$. Peptide coverages were 56 and $74 \%$, respectively. 
The work reported here is one of few examples of multistep reactions implemented by digital microfluidics. This work required close attention to two key phenomena: evaporation and nonspecific adsorption. The former is particularly significant for procedures involving multiple incubation steps, as we observe droplets to evaporate at $\sim 100-1000 \mathrm{~nL} / \mathrm{h}$, depending on the ambient humidity. To counteract this phenomenon, we positioned devices in a humidified chamber during all incubation steps, which eliminated any noticeable evaporation for the duration of experiments. While all the work reported here was performed at room temperature, in other contexts, we have observed this measure to be sufficient for keeping samples hydrated at $37^{\circ} \mathrm{C}$ for $>24 \mathrm{~h}^{71}$ The latter phenomenon, nonspecific adsorption, is a particularly important concern for proteomics, as proteins are highly susceptible to sticking to hydrophobic surfaces (e.g., Teflon-AF-coated DMF devices). In the work reported here, protein sticking was controlled by the use of low concentrations of amphiphilic polymer additives (chapter 2). ${ }^{128}$ In other contexts, we have observed that these additives have little or no effect on biochemical processes ranging from cell vitality ${ }^{71}$ to enzyme kinetics, ${ }^{72}$ and can prevent adsorption even in highly concentrated, heterogeneous solutions such as serum (chapter 2). ${ }^{128}$ The combination of these strategies, that is, incubation in humidified chambers and the use of adhesion-reducing additives, facilitated the development of these digital microfluidic techniques.

\subsubsection{Processing Time}

Fluorogenic assays were used to determine the reaction rates of reduction and digestion by DMF. For reduction, droplets of sample, reductant (TCEP), and fluorogenic dye (ABD-F) were dispensed from reservoirs, merged and actively mixed, and allowed to react at room temperature. ABD-F is non-fluorescent until it reacts with a free sulfhydryl group, and thus can serve as a reporter for reaction progress in reduction of disulfide bonds. ${ }^{131,132}$ Three analytes were evaluated, insulin (3 disulfides), lysozyme (4 disulfides), and BSA (17 disulfides +1 free sulfhydryl group), and representative data are shown in Figure 3.3a. Digestion was evaluated using an intramolecularly quenched, fluorescently labeled protein standard (BODIPY-Casein), which becomes progressively more fluorescent as it is digested. ${ }^{133}$ Representative data for three proteases, trypsin, pepsin, and Lys-C, are shown in Figure 3.3b. DMF-driven reduction and digestion reactions are $90 \%$ complete within 15 - $45 \mathrm{~min}$ and $10-50 \mathrm{~min}$, respectively. This suggests that a full sample processing workup (including protein extraction by precipitation ${ }^{50}$ ) would require approximately $2 \mathrm{~h}$ to complete. This time frame is fast relative to conventional 
methods,${ }^{13}$ but we note that longer times will likely be required for more complex samples (e.g., serum or cell lysate). Regardless, the automation and potential for scaling to parallel sample preparation suggests that the new DMF method will be characterized by improved throughput relative to the manual techniques that are typically used. 
(a)

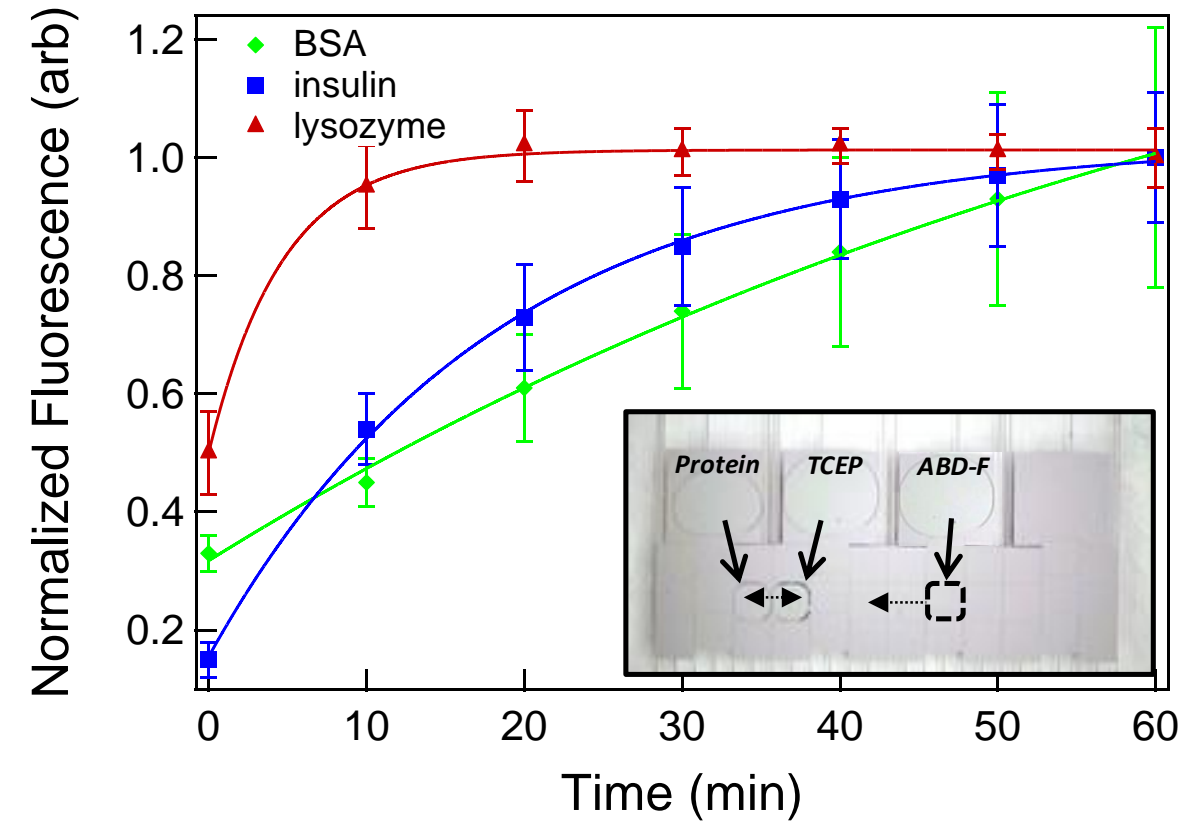

(b)

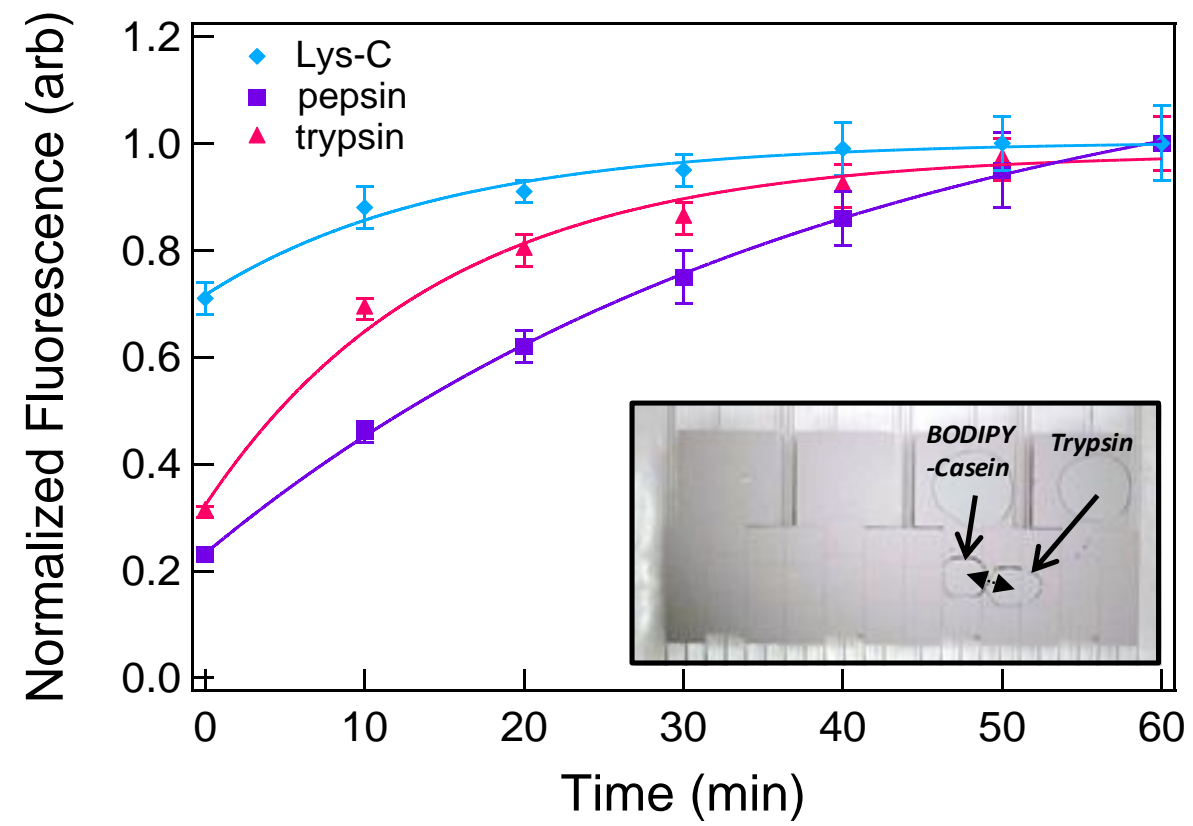

Figure 3.3: Rates of reactions. (a) DMF-driven reduction of BSA $(10 \mu \mathrm{M})$, insulin $(40 \mu \mathrm{M})$ and lysozyme $(40$ $\mu \mathrm{M})$ with reductant, TCEP $(10 \mathrm{mM})$, and ABD-F dye $(12 \mathrm{mM})$ as a fluorogenic reporter. (b) DMF-driven digestion of BODIPY-Casein $(0.33 \mathrm{mg} / \mathrm{mL})$ with Lys-C $(0.07 \mathrm{mg} / \mathrm{mL})$, pepsin $(0.07 \mathrm{mg} / \mathrm{mL})$ and trypsin $(0.07$ $\mathrm{mg} / \mathrm{mL}$ ). Error bars are \pm 1 S.D. 


\subsubsection{Reproducibility}

A critical issue in proteomics is a lack of reproducible, standardized sample handling and processing methods. ${ }^{115}$ We used the fluorogenic assays described above to evaluate the experimental error in DMF-driven on-chip reduction and digestion. As shown in Figure 3.4, the digital microfluidic approach has relatively good precision, with \%RSD ranging from 3-7\% for the reduction assay and $2-5 \%$ for the digestion assay.

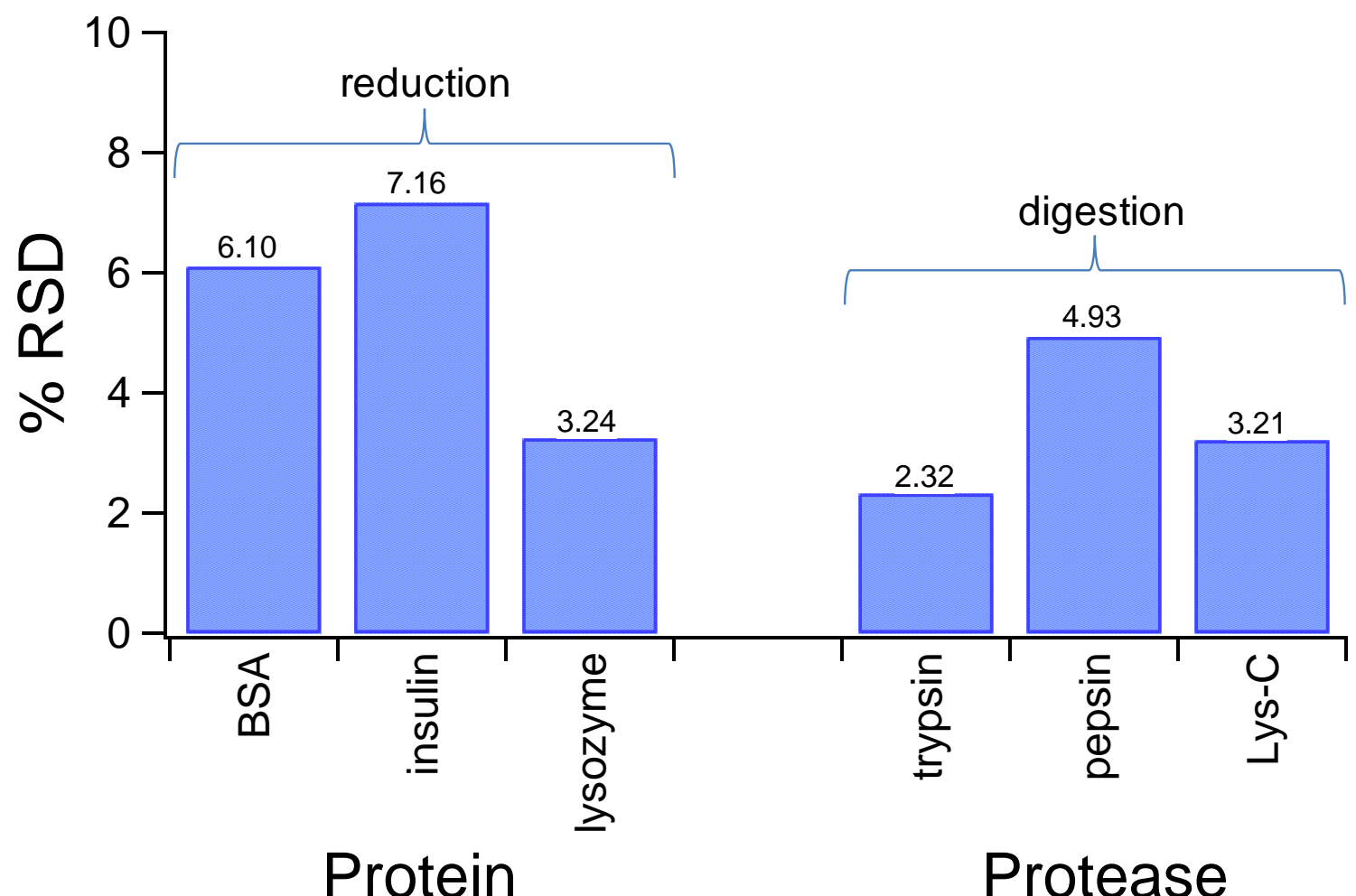

Figure 3.4: Experimental precision in DMF-driven assays. The precision in reduction was evaluated for BSA, insulin and lysozyme (6 picomoles ea.) using TCEP $(10 \mathrm{mM})$ as a reductant and ABD-F $(12 \mathrm{mM})$ as the fluorogenic reporter. The precision in digestion was evaluated using BODIPY-Casein $(0.17 \mu \mathrm{g})$ combined with trypsin $(0.03$ $\mathrm{mg} / \mathrm{mL})$, pepsin $(0.03 \mathrm{mg} / \mathrm{mL})$ and Lys-C $(0.03 \mathrm{mg} / \mathrm{mL})$ proteases. All samples were analyzed in triplicate.

The reduced precision for the reduction assay compared to the digestion is likely caused by the extra dispensing step (three droplets instead of two); we estimate that the \%RSD for dispensing one droplet in these devices is $\sim 1-3 \%$. The dispensing methods used here (i.e., application of driving potentials to 3 electrodes adjacent to a reservoir, resulting in necking and formation of a unit droplet $^{68}$ ) are relatively crude; methods relying on more sophisticated active-feedback control can facilitate higher precision metering. ${ }^{134,135}$ Regardless, the advantage of processing on 
a single substrate is significant, as the multiple containers and transfers inherent in macroscale/manual sample handling can lead to loss of sample and introduction of contaminants such as human keratin. ${ }^{136}$ In addition, if widely adopted, the automation of DMF would eliminate the human error introduced by technicians working in different laboratories. The data in Figure 3.4 demonstrates that digital microfluidics can be used for multistep proteomic processing with high reproducibility. This is an important step forward in our plans to develop a fully integrated proteomic processing platform. Because DMF is compatible with real-world samples (e.g., undiluted serum (chapter 2) ${ }^{50,128}$ ) and large volumes (up to milliliters ${ }^{137}$ ) and can be integrated with separations, ${ }^{138}$ we speculate that this technique may someday contribute to a solution to the well-documented repeatability problems in sample preparation for proteome profiling.

\subsection{Conclusion}

The lack of standardized sample handling and processing in proteomics is a major limitation for the field. Here, we have shown that digital microfluidics (DMF) is a powerful tool for rapid, reproducible, automated sample processing. We propose that DMF has the potential for being useful across a broad range of applications for standardized analysis, as it can be precisely duplicated in any laboratory across the world. 


\section{Chapter 4 - A Tailored Hydrogel Microreactor for Digital Microfluidics: Proteomics Application}

Proteolytic digestion is an essential step in proteomic sample processing. While this step has traditionally been implemented in homogeneous (solution) format, there is a growing trend to use heterogeneous systems, in which the enzyme is immobilized on hydrogels or other solid supports. Here, we introduce the use of immobilized enzymes in hydrogels for proteomic sample processing in digital microfluidic systems. In this technique, pre-formed cylindrical agarose discs bearing immobilized trypsin or pepsin were integrated into digital microfluidic devices. A fluorogenic assay was used to optimize the covalent modification procedure for enzymatic digestion efficiency, with maximum efficiency observed at $31 \mu \mathrm{g}$ trypsin in $2 \mathrm{~mm}$-diameter agarose gel discs. Gel discs prepared in this manner were used in an integrated method in which proteomic samples were sequentially reduced, alkylated, and digested, with all sample and reagent handling controlled by digital microfluidic droplet operation. Mass spectrometry analysis of the products revealed that digestion using the trypsin-gel discs resulted in higher sequence coverage in model analytes relative to conventional homogenous processing. Proof-of-principle was demonstrated for a parallel digestion system in which a single sample was simultaneously digested on multiple gel discs bearing different enzymes. We propose that these methods represent a useful new tool for the growing trend towards miniaturization and automation in proteomic sample processing.

\subsection{Introduction}

This chapter describes a new miniaturized method for eventual application to proteome profiling, the identification of all of the proteins that are present in a biological sample. Proteome profiling is an important research goal, and is being used to identify biomarkers for diagnosis and prognosis of disease, ${ }^{139-141}$ to compare protein expression levels for clinical monitoring of drug efficacy $^{142,143}$ or toxicity ${ }^{142,144,145}$ and may someday be useful for personalized medicine. ${ }^{146}$ Unfortunately, large-scale protein identification is hampered by the complex, multi-step sample processing regimens that are required prior to analysis. For example, proteomic samples are typically digested into smaller peptides by exposure to proteolytic enzymes prior to analysis; this step is widely recognized as being a significant bottleneck for proteome profiling. ${ }^{147}$ In conventional in-solution (homogenous) enzymatic digestion, the enzyme concentration is kept 
low (1:50 enzyme-to-proteomic sample ratio) to avoid autolysis (i.e., self-digestion of enzyme). These low ratios result in lengthy incubation times (often overnight) and require the use of elevated temperatures $\left(37^{\circ} \mathrm{C}\right)$. Furthermore, these conditions lead to an increase in digestion artifacts such as transpeptidation and non-specific cleavage ${ }^{148}$ and deamidation/oxidation, ${ }^{136,149}$ which complicate analysis and reduce confidence in the identification of proteomic analytes.

Many of the disadvantages of homogeneous (in-solution) enzyme digestion can be avoided by using systems in which liquid proteomic samples are exposed to proteolytic enzymes immobilized on solid supports. ${ }^{150}$ In such heterogeneous systems, autolysis is virtually eliminated, such that much higher enzyme-to-substrate ratios can be used, which results in significant improvements in reaction rates. ${ }^{151,152}$ In addition, heterogeneous enzyme digestion systems have advantages of long-term storage stability and resiliency towards heat-induced denaturation and harsh solvents. Many formats exist for heterogeneous digestion systems, including microspin columns, ${ }^{153}$ microspheres/beads, ${ }^{37,154,155}$ polymer monoliths, ${ }^{43,44}$ sol-gel polymers, ${ }^{40,41}$ the inner surfaces of capillary walls, ${ }^{38,156}$ and various types of membranes. ${ }^{46,157}$ But the most promising systems for heterogeneous enzyme digestion are likely those relying on three-dimensional hydrogels as scaffolds for housing immobilized enzymes. ${ }^{158-161}$ Such systems are advantageous for several reasons. First, enzymes are intrinsically fragile and the hydrogel matrix serves as a near-native environment in which enzyme functions are preserved. Second, immobilization strategies for enzymes in hydrogels are typically simple and allow for optimal geometric congruence between the enzyme and solid support. Third, the high porosity of hydrogels allows for immobilization of very high densities of enzyme. And fourth, hydrogel supports can be easily molded to various shapes and sizes tailored to a specific application.

We recently reported a new tool for lab-on-a-chip systems: the combination of hydrogels and digital microfluidics (DMF). ${ }^{162}$ Here, we report the application of this tool to proteolytic digestion for proteome profiling, and demonstrate its superiority to conventional techniques. DMF is a fluid-handling technique in which liquid samples are manipulated on the surface of an array of electrodes by electrostatic forces, ${ }^{122}$ and has been applied to a variety of applications related to proteomics including sample purification by precipitation, ${ }^{50}$ reduction of disulfides (chapter 3), ${ }^{4,52}$ alkylation of thiols (chapter 3), ${ }^{49,51,52}$ proteolytic digestion (chapters 2 and $3),{ }^{49,51,52,128}$ and combinations thereof. ${ }^{163}$ These previous reports represent important steps towards the goal of integrated proteome profiling, but they are limited by the challenges inherent 
to homogeneous digestions described above. We speculate that the new techniques presented here, which combine the fluid manipulation and system integration capabilities of digital microfluidics with the rapid and efficient reaction rates afforded by heterogeneous hydrogel microreactors will be a useful addition to the growing trend in miniaturization of proteomic analysis tools, ${ }^{117}$ and may eventually contribute to a new generation of methods for automated proteome profiling.

\subsection{Materials and Method}

\subsubsection{Reagents and Material}

Bovine serum albumin (BSA), lysozyme from chicken egg white, insulin from bovine pancreas, trypsin from bovine pancreas, pepsin from porcine gastric mucosa, iodoacetamide (IAM), tris$\mathrm{HCl}$, sodium acetate, acetic acid, pluronic F127, fluorinert FC-40, acetonitrile (ACN), trifluoroacetic acid (TFA), fluorescein isothiocyanate (FITC), fluorescamine, and low melting point agarose were purchased from Sigma Aldrich Canada (Oakville, ON). Tris-(2carboxyethyl)phosphine hydrochloride (TCEP) and E6638 EnzChek® Protease Assay Kit were purchased from Life Technologies/Invitrogen (Burlington, ON). Ziptip ${ }_{\mathrm{C} 18}{ }^{\circledR}$ pipette tips were purchased from Millipore (Etobicoke, ON), and $\alpha$-cyano-4-hydroxycinnamic acid ( $\alpha$-CHCA) was purchased from Waters Limited (Mississauga, ON). Deionized (DI) water had a resistivity of $18 \mathrm{M} \Omega \bullet \mathrm{cm}$ at $25^{\circ} \mathrm{C}$. Unless otherwise indicated, all protein and processing reagent solutions were prepared in working buffer $(50 \mathrm{mM}$ tris- $\mathrm{HCl}, \mathrm{pH} 7.8,0.08 \%$ w/v Pluronic F127 in DI water) immediately prior to use.

\subsubsection{DMF Device Fabrication and Operation}

Digital microfluidic devices were fabricated in the University of Toronto Emerging Communications Technology Institute (ECTI) cleanroom facility, using a transparent photomask printed at Pacific Arts and Design (Markham, ON). Digital microfluidic device bottom plates bearing patterned chromium electrodes were formed by photolithography and etching as described previously ${ }^{164}$ and were coated with $15 \mu \mathrm{m}$ of Parylene-C and $200 \mathrm{~nm}$ of Teflon-AF. Parylene-C was applied using a vapor deposition instrument (Specialty Coating Systems), and Teflon-AF was spin-coated (1\% wt/wt in Fluorinert FC-40, $1600 \mathrm{rpm}, 60 \mathrm{~s})$ followed by postbaking on a hot-plate $\left(160^{\circ} \mathrm{C}, 10 \mathrm{~min}\right)$. The polymer coatings were removed from contact pads 
by gentle scraping with a scalpel to facilitate electrical contact for droplet actuation. In addition to patterned DMF device bottom plates, unpatterned glass microscope slides and DMF device top plates formed from indium tin oxide (ITO) coated glass substrates (Delta Technologies Ltd, Stillwater, MN) were coated with Teflon-AF (200 nm, as above).

The device used here featured an array of 68 square actuation electrodes $(2 \times 2 \mathrm{~mm}$ ea. $)$ connected to 10 reservoir electrodes $(5 \times 5 \mathrm{~mm}$ ea.), with inter-electrode gaps of 50-100 $\mu \mathrm{m}$. Devices were assembled with an unpatterned ITO-glass top plate and a patterned bottom plate separated by a spacer formed from 2 pieces of double-sided tape (total spacer thickness $140 \mu \mathrm{m}$ ). To actuate droplets, driving potentials $\left(200-250 \mathrm{~V}_{\mathrm{pp}}\right)$ were generated by amplifying the output of a function generator (Agilent Technologies, Santa Clara, CA) operating at $18 \mathrm{kHz}$. Droplets were sandwiched between two plates and were actuated by applying driving potentials between the top electrode (ground) and sequential electrodes on the bottom plate via the exposed contact pads. Droplet actuation was monitored and recorded by a CCD camera mounted on a lens.

\subsubsection{Hydrorogel Disc Fabrication}

Low-melting point agarose was dissolved in DI water to a concentration of $4.0 \mathrm{wt} \%$ by incubating the mixture in an oven at $70^{\circ} \mathrm{C}$ for $1 \mathrm{~h}$. Some mixtures included $10 \mu \mathrm{m}$ diameter polystyrene beads (Sigma Aldrich Canada) to make the gels easier to visualize. After removing the mixture from the oven, $2 \mu \mathrm{L}$ aliquots were pipetted and sandwiched between two unpatterned Teflon AF-coated glass slides held together with 2 pieces of double-sided tape as a $140 \mu \mathrm{m}$ spacer. These assemblies were placed on a cold pack to allow the droplets to gel $(\sim 2 \mathrm{~min})$. The resulting gel discs were $\sim 1 \mathrm{~mm}$ in diameter and $140 \mu \mathrm{m}$ height $(\sim 440 \mathrm{~nL})$ and could be retrieved from the glass-slide assembly using tweezers. For use with digital microfluidics, gel discs were sandwiched between a bottom plate and a top plate, with each disc positioned such that it straddled the interface between two electrodes on the bottom plate.

\subsubsection{Covalent Attachment of Enzymes to Gels}

Enzymes were covalently attached to agarose gel discs using methods similar to those developed by Guisan and coworkers, ${ }^{165-167}$ in four steps. In step (i), each agarose gel disc was suspended in $2.4 \mu \mathrm{L}$ DI water. $680 \mathrm{~nL}$ aqueous sodium borohydride $(28 \mathrm{mg} / \mathrm{mL}$ in $1.7 \mathrm{~N} \mathrm{NaOH})$ was then added to the suspension. The reaction vessel was placed on an ice bath followed by an addition 
of $134 \mathrm{~nL}$ glycidol. The reaction was incubated overnight (18 h, RT). Afterwards, the gels were washed with DI water and filtered using a Buchner funnel. In step (ii), each gel was suspended in $18 \mu \mathrm{L}$ of an aqueous solution of sodium periodate (typically $3.5 \mathrm{mM}$; see next section for details) for 75 minutes followed by the same wash and filtration step as above. In step (iii), each gel was then suspended in $18 \mu \mathrm{L}$ of an enzyme solution $(4.4 \mathrm{mg}$ trypsin/mL in $50 \mathrm{mM}$ sodium bicarbonate, $\mathrm{pH} 10$, or $4.4 \mathrm{mg}$ pepsin/mL in $50 \mathrm{mM}$ sodium acetate, $\mathrm{pH} 4.5$ ) where it was incubated (72 h, RT). Finally in step (iv), $2 \mu \mathrm{L}$ aqueous sodium borohydride $(10 \mathrm{mg} / \mathrm{mL})$ was added to the suspension and incubated for 30 minutes (RT). The gels were isolated using a wash and filtration step as described above and stored at $4^{\circ} \mathrm{C}$ until use.

To evaluate the uniformity of covalent attachment of enzymes to gel discs, FITC-labeled trypsin was prepared by combining $1 \mathrm{~mL}$ of $2 \mathrm{mg} / \mathrm{mL}$ trypsin solution (in $0.1 \mathrm{M}$ sodium carbonate buffer, $\mathrm{pH}$ 9) with $50 \mu \mathrm{L}$ of $1 \mathrm{mg} / \mathrm{mL}$ FITC solution (in anhydrous DMSO). The reaction mixture was incubated for at least 8 hours in the dark $\left(4^{\circ} \mathrm{C}\right)$. Gels modified with this dye-labeled trypsin were formed as above, and were evaluated using a Leica TCS SP2 scanning confocal microscope (Leica Microsystems Canada, Richmond Hill, ON) equipped with an $\mathrm{Ar}^{+}$laser (488 $\mathrm{nm})$ with a $4 \times$ objective lens. Fluorescence was passed through a 510-525 nm band-pass filter, and digital images were acquired using Lecia Confocal acquisition software. Images were collected at $10 \mu \mathrm{m}$ increments to from bottom $(0 \mu \mathrm{m})$ to the top $(140 \mu \mathrm{m})$ of the gel discs.

\subsubsection{Gel Disc Microreactor Optimization}

To determine the optimum density of enzyme molecules for proteolytic digestion, trypsinlabelled gels were formed as above, with exposure to $0.5,2,3.5,5.5$, or $7.5 \mathrm{mM}$ sodium periodate in step (iii). Each gel was placed onto a DMF device. A $2.5 \mu \mathrm{L}$ droplet of quenched bodipy-casein $(100 \mu \mathrm{g} / \mathrm{mL}$ in working buffer) from an EnzChek® Protease Assay Kit was dispensed and merged onto the gel and moved back and forth across the gel for a few seconds. The device was placed in a humidified chamber (a Petri dish partially filled with DI water) and incubated for 1 hour (RT). After incubation, the droplet was dispensed from the gel, $2 \mu \mathrm{L}$ was collected by pipet and deposited in a multiwell plate, and was diluted to $50 \mu \mathrm{L}$ with DI water. A PHERAstar multiwell plate reader (BMG Labtech, Durham, NC) was used to measure the fluorescence $\left(\lambda_{\mathrm{ex}}=485 \mathrm{~nm} ; \lambda_{\mathrm{em}}=520 \mathrm{~nm}\right.$; focal height $=7.0 \mathrm{~mm}$; gain $\left.=300\right)$ of digested BODIPY-Casein. Three replicates were carried out per condition. Gels exposed to $3.5 \mathrm{mM}$ 
sodium periodate were found to have the highest digestion efficiency, and thus this condition was used for all subsequent experiments.

The amount of trypsin attached to gel discs under the optimal conditions described above (with $3.5 \mathrm{mM}$ sodium periodate) was determined using a fluorogenic depletion assay. Gel discs were treated as in steps (i)-(iii) above, and after incubation, $15 \mu \mathrm{L}$ of the supernatant (containing unbound trypsin) was collected by pipet and deposited in a multiwell plate and combined with $5 \mu \mathrm{L}$ of $3 \mathrm{mg} / \mathrm{mL}$ fluoresamine in acetone. This solution was diluted to $50 \mu \mathrm{L}$ with DI water and evaluated using a PHERAstar multiwell plate reader $\left(\lambda_{\mathrm{ex}}=355 \mathrm{~nm} ; \lambda_{\mathrm{em}}=460 \mathrm{~nm}\right.$; focal height $=$ $7.0 \mathrm{~mm}$; gain = 50). The average value of fluorescence intensity of the supernatant from three gels was compared to a calibration curve of fluorescamine-derivatized-trypsin (range: 0.275$4.40 \mathrm{mg} / \mathrm{mL}$ ) to determine the amount of un-bound trypsin after reaction with the gels. The amount of trypsin bound to the gels was assumed to be the difference between the initial concentration $(4.4 \mathrm{mg} / \mathrm{mL})$ and the concentration in the supernatant after incubation with gels.

\subsubsection{Proteomic Workup and Mass Spectrometry}

Proteomic sample workup proceeded in three steps: (1) reduction - one $600 \mathrm{~nL}$ droplet of analyte (BSA or lysozyme, $1 \mathrm{mg} / \mathrm{mL}$ in working buffer) and a second $600 \mathrm{~nL}$ droplet of TCEP (10 mM in working buffer) were dispensed and merged by DMF followed by incubation (60 min, RT); (2) alkylation - a third $600 \mathrm{~nL}$ droplet of iodoacetamide $\left(12 \mathrm{mM}\right.$ in $\left.\mathrm{dH}_{2} \mathrm{O}\right)$ was dispensed and merged with the combined droplet of analyte/TCEP and incubated again (room temp., dark, 45 min); (3) tryptic digestion. The third step was implemented in one of three different conditions. In condition (i) (named "SOLUTION-LOW"), a $600 \mathrm{~nL}$ droplet of trypsin at $0.02 \mathrm{mg} / \mathrm{mL}$ in working buffer was dispensed and merged with the combined droplet of analyte/TCEP/iodoacetamide. In condition (ii) (named "IN-GEL"), the combined droplet analyte/TCEP/iodoacetamide was actuated onto a gel disc containing trypsin. In condition (iii) (named "SOLUTION-HIGH"), a $600 \mathrm{~nL}$ droplet of trypsin at $40 \mathrm{mg} / \mathrm{mL}$ in working buffer was dispensed and merged with the combined droplet of analyte/TCEP/iodoacetamide. In all cases, the digestion mixture was allowed to incubate $(4 \mathrm{~h}, \mathrm{RT})$ in a humidified chamber (a Petri dish partially filled with DI water) to limit evaporation. Samples on gels were dispensed away from the gels, and all samples were then quenched by dispensing and actuating a $600 \mathrm{~nL}$ droplet of 
$2.5 \%$ TFA to merge with the droplet. At least three replicate samples were evaluated for each condition.

In some cases, a modified IN-GEL procedure was used, in which the droplet at the end of step 2 (a mixture of analyte, TCEP, and iodoacetamide) was split into two separate droplets (each 900 $\mathrm{nL}$ ) which were individually actuated onto two different gel discs. One gel disc contained trypsin and the other contained pepsin. The droplets were allowed to incubate in a humidified chamber and were then quenched as above.

After processing by DMF, each sample was purified using a Ziptip ${ }_{\mathrm{C} 18}{ }^{\circledR}$ and then analyzed by matrix assisted laser desorption/ionization mass spectrometry (MALDI-MS). Briefly, Ziptip ${ }_{\mathrm{C} 18}{ }^{\circledR}$ were wetted in $100 \% \mathrm{ACN}$ containing $0.1 \% \mathrm{TFA}(3 \times)$ and then equilibrated in $5 \% \mathrm{ACN}$ containing $0.1 \%$ TFA $(3 \times)$. The quenched digest samples generated by DMF were drawn in and out of tips for $7-10$ cycles, and then washed with $5 \%$ ACN containing $0.1 \%$ TFA (3x). Finally, samples were eluted in $100 \%$ ACN containing $0.1 \%$ TFA $(2 \mu \mathrm{L})$ onto a stainless steel MALDI target plate. A $1 \mu \mathrm{L}$ matrix solution $\left(10 \mathrm{mg} / \mathrm{mL} \alpha-\mathrm{CHCA}\right.$ in 50:50 ACN: $\mathrm{dH}_{2} \mathrm{O}$ containing $0.1 \%$ TFA) was added to each sample. After drying, spots were analyzed using a Micro-MX mass spectrometer (Waters, Milford, MA) operating in positive and reflectron modes over a mass-tocharge ratio $(\mathrm{m} / \mathrm{z})$ range from 600 - 2000. At least one hundred shots were collected per spectrum, with laser power tuned to optimize the signal to noise ratio $(\mathrm{S} / \mathrm{N})$. Data were processed by normalization to the largest analyte peak, baseline subtraction, and smoothed with a 15-point running average. Spectra of enzyme digests were evaluated visually.

To characterize digested peptides in mass spectra, peaks with intensities below $10 \%$ and $2.5 \%$ signal relative to the largest peak intensity were rejected for single and double enzyme digestion experiments, respectively. The masses of expected peaks were determined using the PeptideMass prediction/characterization tool on the ExPASy proteomic server taking protein sequences from the UniProt Knowledgebase (Swiss-Prot and TrEMBL), with 1 allowed missed cleavages, cysteines treated with iodoacetamide and a mass accuracy of +/- 1.2 Da. 


\subsection{Results and Discussion}

\subsubsection{Gel Disc Microreactor Formation and Use}

Fiddes et al. ${ }^{162}$ recently introduced a new model for miniaturized systems: the marriage of hydrogel discs and digital microfluidics. Here we applied this combination to implement a critical step in many proteomic applications, enzymatic digestion. Using an adaptation of methods reported previously, ${ }^{158,165-167}$ agarose gel discs were modified to bear proteolytic enzymes (trypsin or pepsin), such that when droplets containing proteomic analytes were exposed to the modified discs, the proteins were enzymatically cleaved.

Figure 4.1 illustrates the mechanism by which enzymes are immobilized on gel discs. In step (i), pre-formed agarose discs $(\mathrm{Agr}-\mathrm{OH})$ are exposed to an activating agent, glycidol (2,3epoxypropanol), to form an intermediate, glyceryl-agarose (Agr-O- $\left.\mathrm{CH}_{2}-\mathrm{CHOH}-\mathrm{CH}_{2} \mathrm{OH}\right)$, which is then oxidized in step (ii) with sodium periodate to form glyoxyl-agarose (Agr$\mathrm{CH}_{2} \mathrm{CHO}$ ). Subsequent exposure of the oxidized gel in step (iii) to an enzyme solution results in the covalent attachment of the enzyme via a Schiff's base reaction. Finally in step (iv), a reduction in sodium borohydride transforms the weak Schiff's bases into stable secondary amide bonds and all remaining aldehyde on the solid support into inert hydroxy groups. The inset of Figure 4.1 contains a z-stack series of cross-sectional confocal images of a gel disc that was modified with FITC-trypsin. The images reveal that the enzyme is distributed throughout the gel, which indicates that a large surface area is used for enzymes immobilization.

Gel-disc microreactors are easily integrated onto DMF platforms by placing them directly onto the area/electrode of interest. Gel discs on digital microfluidic devices remain stationary, such that liquid droplets can be manipulated relative to them. Figure 4.2 demonstrates the process for a proteolytic microreactor. A droplet containing a protein sample is delivered to the gel and is then incubated such that the covalently bound proteolytic enzyme can digest the sample into peptides. After incubation, the droplet (now containing digest-peptides) can be dispensed away from the gel for further processing or analysis. 
(i)
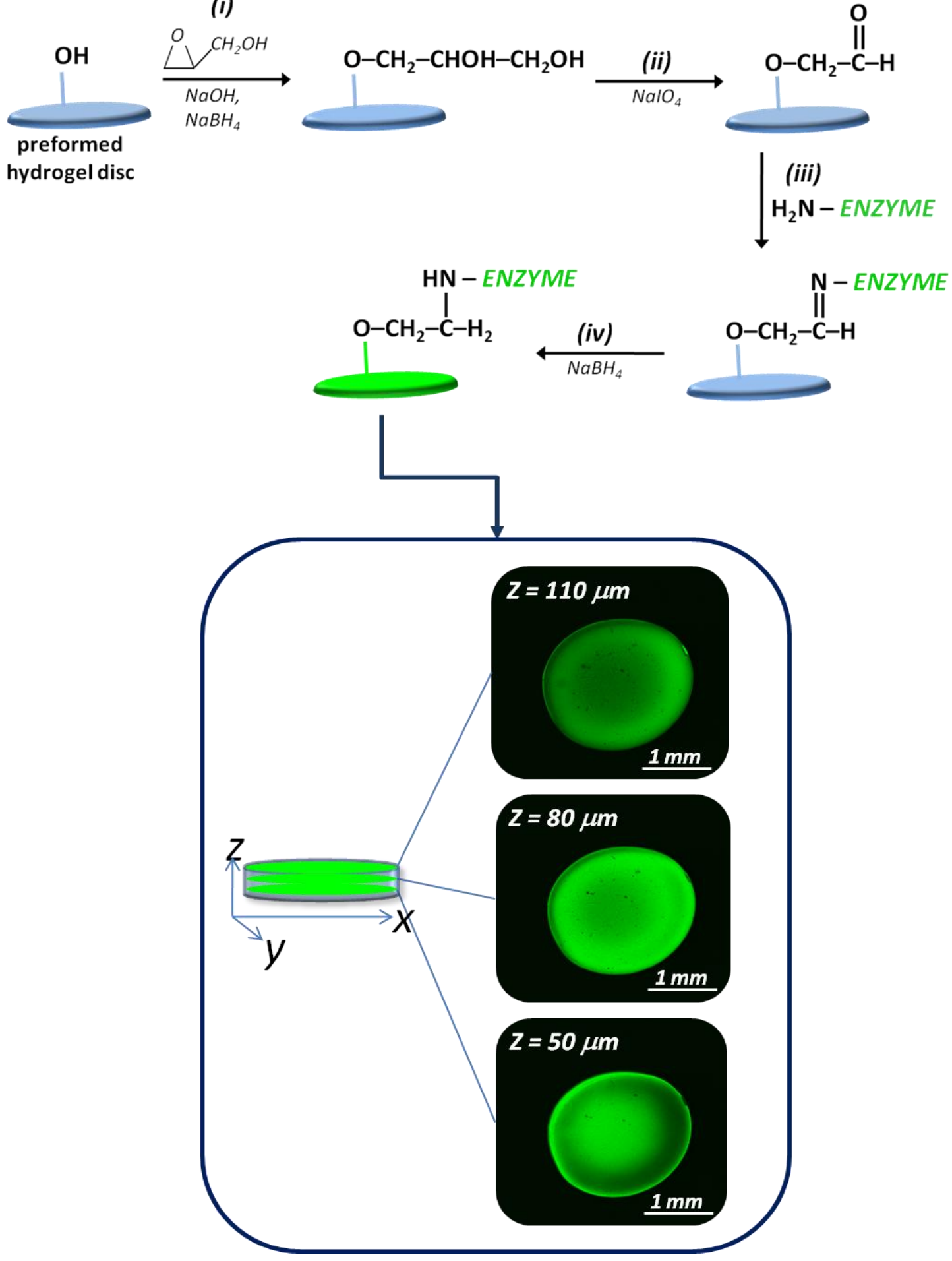

Figure 4.1: Immobilization of enzymes onto hydrogel discs. Reaction scheme (top): (i) preformed agarose discs $(\mathrm{Agr}-\mathrm{OH})$ are activated with glycidol (2,3-epoxypropanol) to form an intermediate glyceryl-agarose $\left(\mathrm{Agr}-\mathrm{O}-\mathrm{CH}_{2}-\right.$ $\mathrm{CHOH}-\mathrm{CH}_{2} \mathrm{OH}$ ); (ii) glyceryl-agarose is oxidized with periodate to generate glyoxyl-agarose (Agr- $\left.\mathrm{CH}_{2} \mathrm{CHO}\right)$; (iii) enzyme molecules are coupled to the aldehyde groups via the Schiff base reaction; (iv) reduction with sodium borohydride yields an enzyme-agarose derivative. Z-stack of cross-sectional fluorescent confocal images (bottom) of FITC-labeled trypsin attached to hydrogel discs. The scale bar is $1 \mathrm{~mm}$. 
(a)

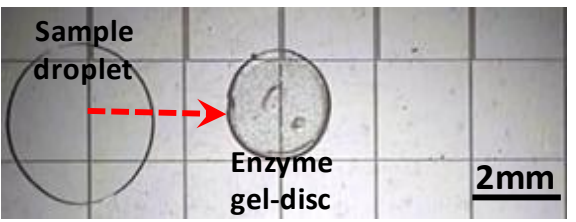

(b)

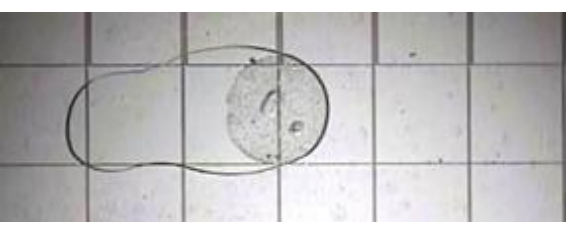

(c)

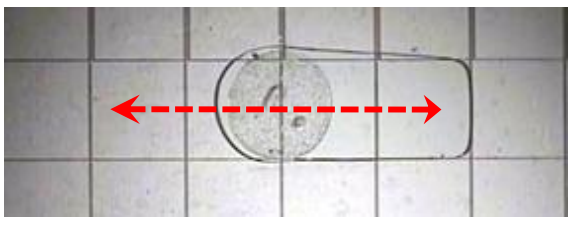

(d)

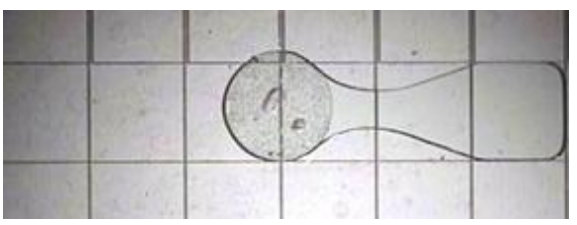

(e)
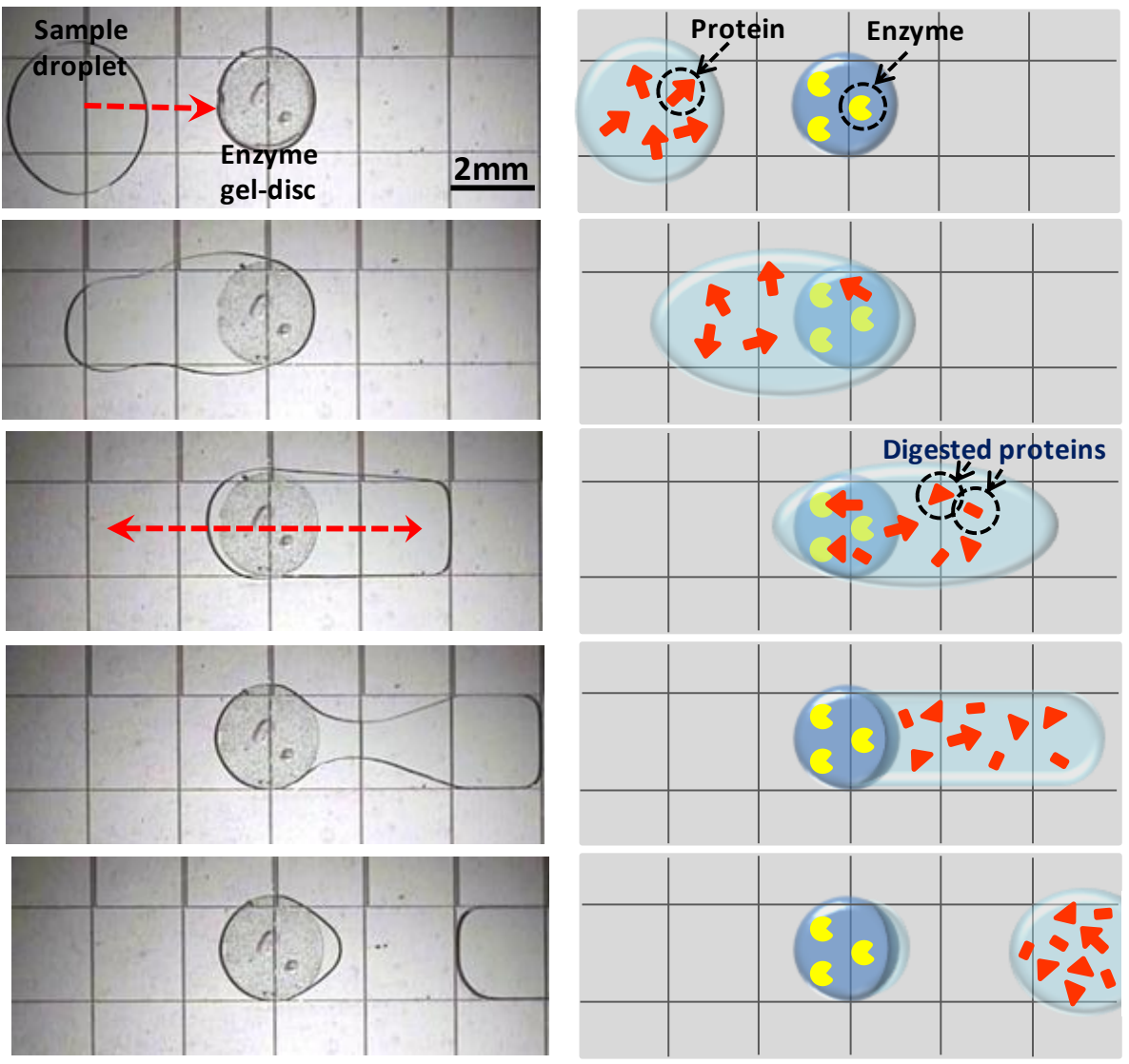

Figure 4.2: Hydrogel proteolytic enzyme microreactors. A series of images from a movie (left) and a schematic (right) depicts a typical digestion in a $2 \mathrm{~mm}$ dia. gel disc on a DMF device. A $2 \mu \mathrm{L}$ droplet containing a proteomic sample is delivered to the gel (a, b), actively incubated (c), dispensed from the gel (d), and then isolated (e). The gel disc contains a suspension of $10 \mu \mathrm{m}$ dia. beads to make it visible.

\subsubsection{Gel Disc Microreactor Optimization}

For gel disc microreactors, the density of immobilized enzymes determines the overall reaction efficiency. If the density is too low, the small number of catalytic sites will contribute to a slow reaction rate, while if the density is too high, the enzyme molecules can sterically hinder each other, which can also contribute to sub-optimal rates. To determine the optimum density of enzymes for the current application, we evaluated a series of different trypsin densities on gel discs.

The critical step that determines eventual enzyme density in the process reported here is the number of aldehyde groups generated on the gel (step ii in Figure 4.1). A series of gels were 
prepared bearing different densities of aldehyde moieties by exposure to different concentrations of sodium periodate. These gels were then modified with trypsin, incorporated onto DMF devices, then exposed to droplets (as in Figure 4.2) containing quenched BODIPY-casein, a reporter that becomes progressively more fluorescent as it is digested. The data from the assay is shown in Figure 4.3. As shown, exposure of gels to $3.5 \mathrm{mM}$ sodium periodate resulted in the highest signal, which correlates to the highest rate of digestion. This condition was used for all subsequent experiments. The density of trypsin molecules attached under these conditions was determined using a fluorogenic depletion assay to be $31 \pm 4 \mu \mathrm{g}$ trypsin per $2 \mathrm{~mm}$ dia. gel disc.

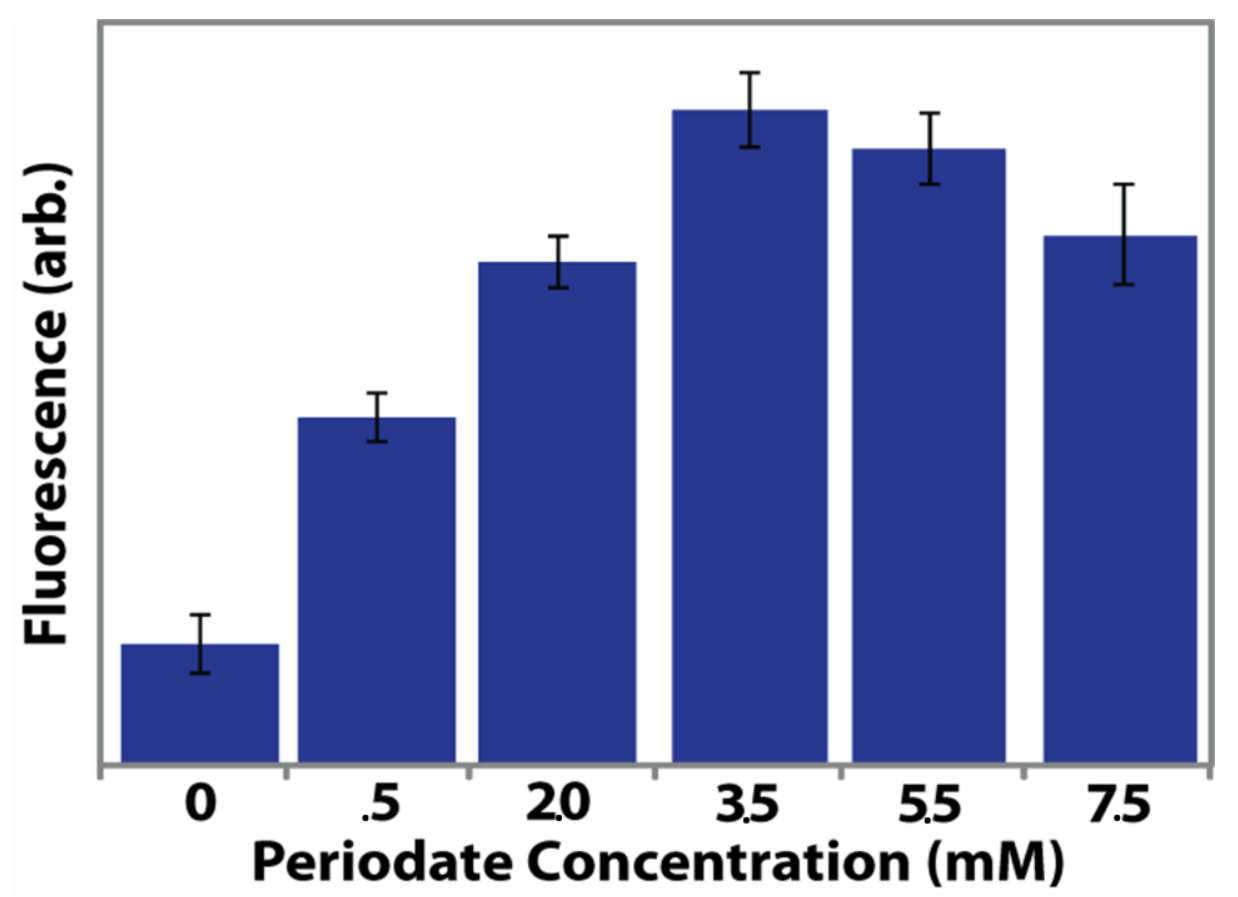

Figure 4.3: Gel disc microreactor optimization. Gel discs were exposed to various concentrations of $\mathrm{NaIO}_{4},(0.5,2$, $3.5,5.5$, or $7.5 \mathrm{mM}$ ), which determines the density of enzyme immobilized. A droplet of a fluorogenic reporter, quenched bodipy-casein (which becomes progressively more fluorescent as it is digested) was dispensed, merged onto the gel, incubated, and then collected for determination of fluorescence intensity. The data represent three replicates per condition, with error bars of $\pm 1 \mathrm{SD}$. The amount of trypsin immobilized in the optimal conditions (i.e., after exposure of gel discs to $3.5 \mathrm{mM}$ sodium periodate) was found to be $31 \pm 4 \mu \mathrm{g}$ trypsin per $2 \mathrm{~mm}$ dia. gel disc.

\subsubsection{Comparison of In-Gel and In-Solution Digestion Quality}

Digital microfluidics has been touted as being a useful new tool for proteomic sample preparation, particularly for implementing proteolytic digestion (chapters 2 and 3). ${ }^{49-52,128,163}$ But until now, all enzyme digestion methods implemented by digital microfluidics have been homogeneous (i.e., solution only). As noted in the introduction, there are significant benefits to 
using heterogeneous digestion systems (i.e., those with proteolytic enzymes immobilized on a solid substrate). We hypothesized that the new digital microfluidic methods with gel microreactors reported here would benefit from these same advantages. To test the hypothesis, we evaluated three conditions: proteins digested on a gel disc microreactor (named "IN-GEL"), proteins digested in solution at 1:50 enzyme:protein concentration (named "SOLUTION-LOW") and proteins digested in solution at 40:1 enzyme:protein concentration (named "SOLUTIONHIGH"). In all cases, the samples were reduced, alkylated and then digested, with all reagent manipulation steps implemented by digital microfluidic droplet actuation.

MALDI-MS was used to evaluate the quality of the digestions generated by each of the conditions. Figure 4.4 shows representative spectra (normalized to the highest peak intensity) of processed (a) BSA and (b) lysozyme collected using the three digestion protocols. In each of these experiments, the digesting samples were allowed to incubate for four hours at room temperature. The SOLUTION-LOW condition is the enzyme-to-protein ratio that is conventionally used for proteomic applications; although this ratio results in slow digestions, it is used to circumvent the appearance of autolysis peaks. As shown, the SOLUTION-LOW condition resulted in reasonable spectral quality, but the spectra contained many peaks that could not be identified, which likely originated from partially digested peptides. In contrast, in the spectra generated by the IN-GEL condition, almost all of the major peaks were identified as digest peptides, indicating that the digestions were substantially complete. This is notable, given that the digestion was carried out for relatively short time and at room temperature.

As a second test for the new system, a SOLUTION-HIGH condition was tested, which is homogeneous, but contains a similar amount of enzyme as the IN-GEL system. As shown, the spectra generated using this condition were dominated by autolysis peaks, with few peptide peaks originating from the analytes. This suggests that the improved performance of the IN-GEL condition is a function both of the high concentration of the enzyme and the benefits provided from the immobilization of enzymes on gels. 
(a) BSA

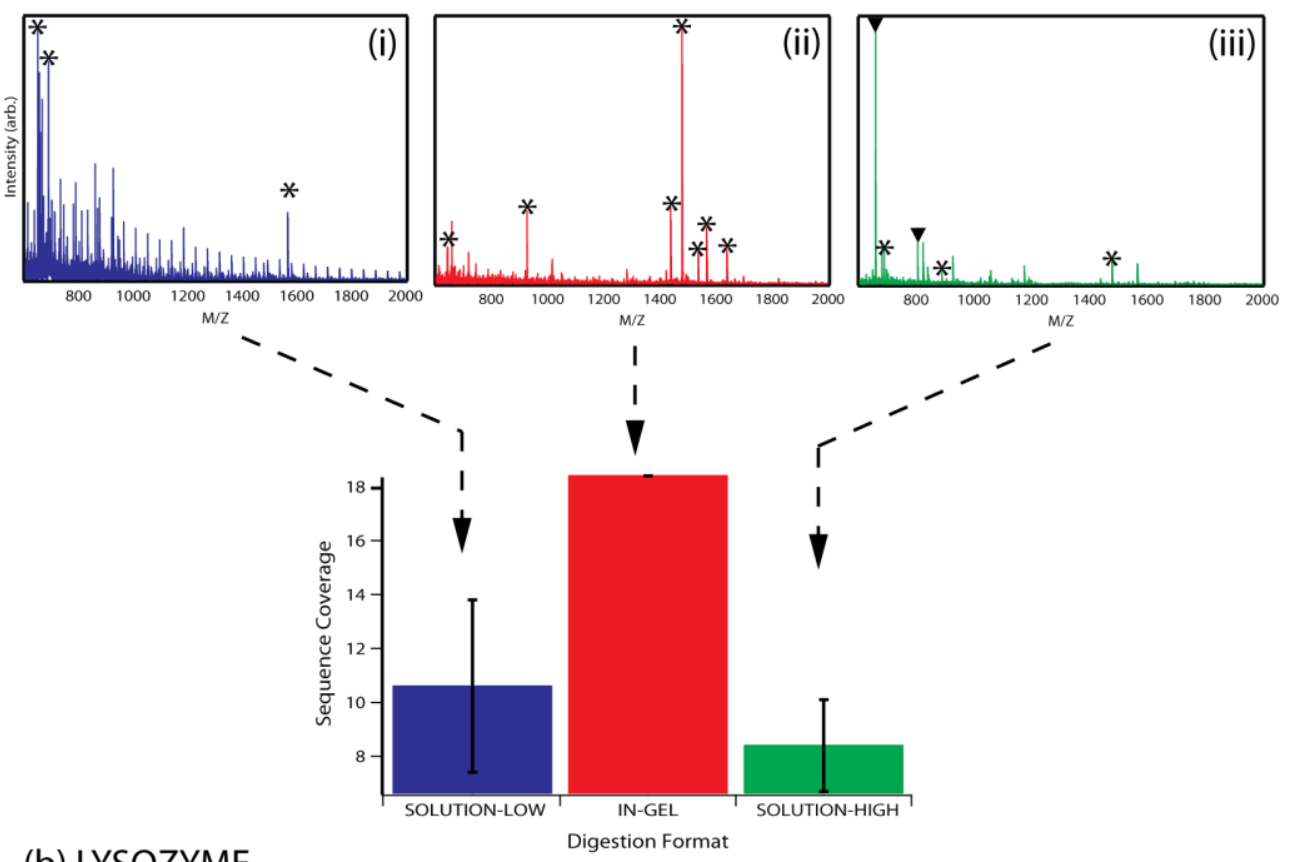

(b) LYSOZYME

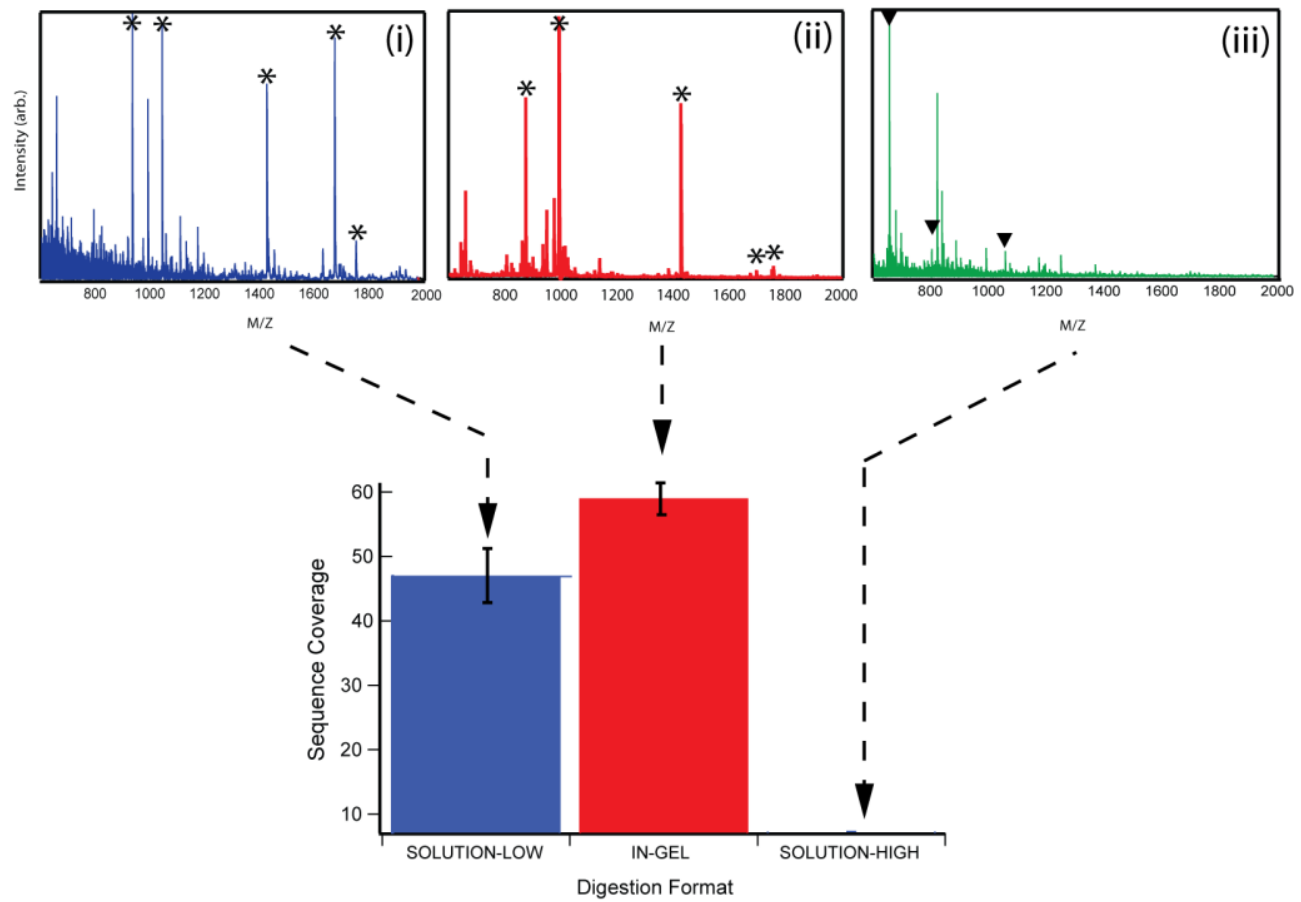

Figure 4.4: Comparison of digestion efficiency. Representative MALDI-MS spectra (top) and their corresponding sequence coverage data (bottom) for (a) BSA and (b) lysozyme digested (i) in-solution with low trypsin concentration $(0.02 \mathrm{mg} / \mathrm{mL})$, (ii) in-gel and (iii) in-solution with high trypsin concentration $(40 \mathrm{mg} / \mathrm{mL})$. Asterisks $\left.{ }^{*}\right)$ in the spectra denote tryptic peptides of the analytes, and upside down triangles $(\nabla)$ denote tryptic autolysis peaks. The sequence coverage data represent three replicates $(n=3)$ per condition, with error bars of \pm 1 S.D. 


\subsubsection{Multiplexed Digestion}

For complex samples, the list of peptides formed after digestion with a single enzyme is often not sufficient to identify the proteins in question. For this reason, parallel digestions using different enzymes can improve sequence coverage by identifying more peptides resulting in increases confidence in protein identification in proteome profiling. ${ }^{168,169}$ Digital microfluidics seems well-suited to such tasks, given that droplets containing samples can be manipulated, split, and delivered to multiple locations in a device in parallel. To test this idea, we developed a parallel digestion scheme in which an initial droplet was reduced and alkylated, and was then split and delivered to two different gel microreactors -- one containing trypsin, and one containing pepsin. The process is shown in Figure 4.5. MALDI-MS results generated from the two different gels reveals a large number of unique tryptic and peptic peptides. An analysis of the peptides identified confirms that the sequence coverage from both enzymes $(34 \%)$ is indeed higher than that of either enzyme alone (19\% and 19\% for trypsin and pepsin, respectively). These results illustrate the potential for parallel proteomic sample processing for digital microfluidics paired with hydrogel discs. We propose that this capacity may eventually prove to be a useful part of an automated platform for proteome profiling.

\subsection{Conclusion}

Here, we demonstrated the utility in combining digital microfluidics and hydrogels discs for proteomics applications. Hydrogel discs bearing covalently attached proteolytic enzymes were found to be useful for proteomic sample preparation, with better performance than conventional homogeneous techniques. We propose that these methods represent a useful new tool for the growing trend towards miniaturization and automation in proteomic sample processing. 


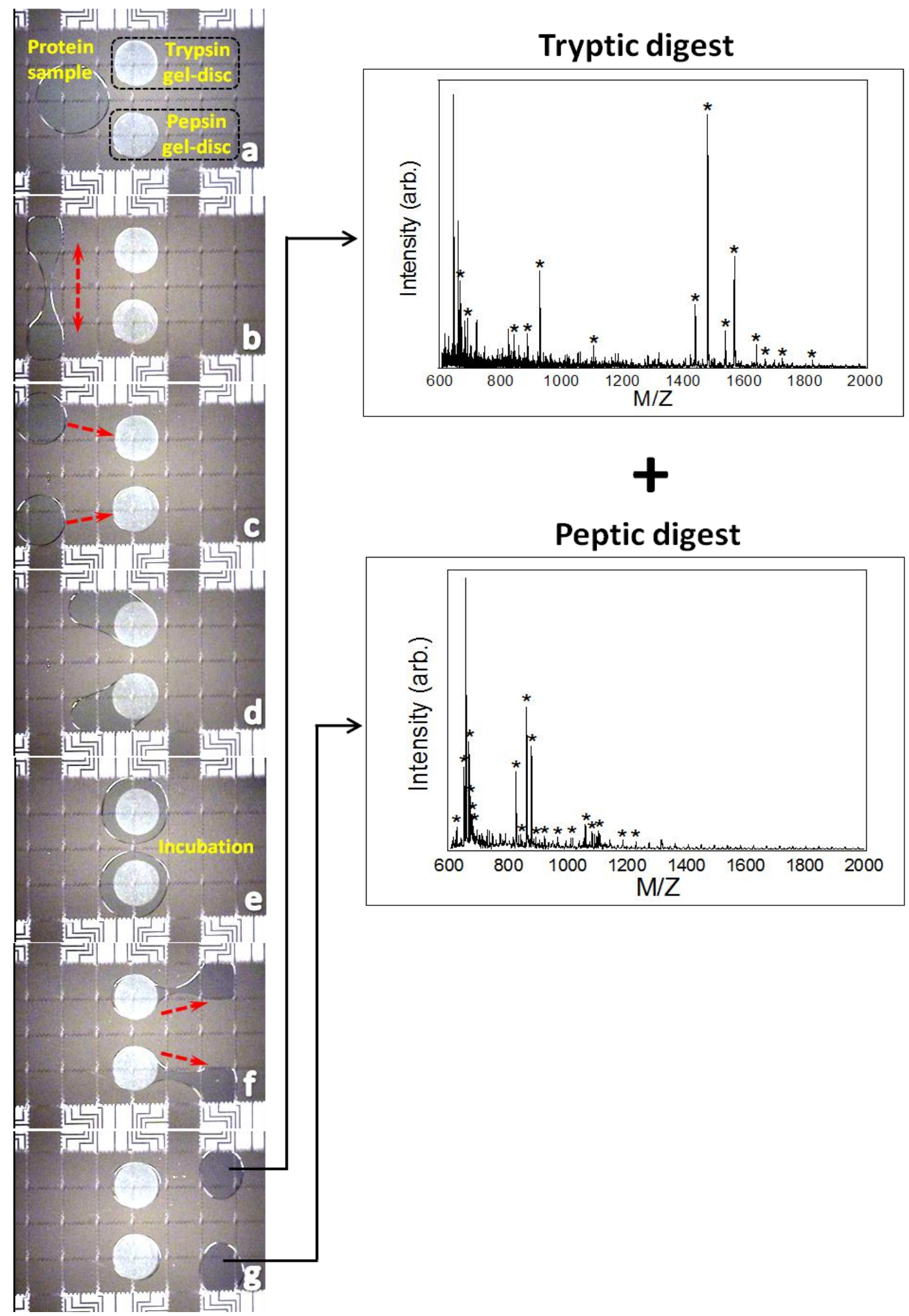

Figure 4.5: DMF-driven multi-enzyme digestion of a protein sample using hydrogel discs. Frames from a movie (left) depicting a droplet containing BSA being split into two daughter droplets (a-c) which are then delivered to hydrogel discs bearing trypsin (top) and pepsin (bottom). Samples were actively incubated (d,e) and then isolated (f,g) for analysis by MALDI-MS. The gel discs contain a suspension of $10 \mu \mathrm{m}$ dia. beads to make them visible. Representative MALDI-MS spectra from three replicate experiments (right) of tryptic and peptic digests. Asterisks $(*)$ denote tryptic and peptic peptides originating from BSA. 


\section{Towards a Fully Integrated Microfluidic System for Front-end Proteomic Processing}

Conventional proteomic sample preparation and processing introduce pre-analytical variations that pose major challenges in the analysis of the proteome. Digital Microfluidics (DMF) has been touted as a potential platform for rapid, automated solution-phase reactions with the great promise as a standardized platform for laboratory science. Here, we report a DMF-based method integrating six common processing steps in proteomics, including protein extraction, clean-up, resolubilization, reduction, alkylation, and enzymatic digestion. In initial work, the reproducibility of the method was evaluated using Spectral Counting, with the goal of eventual optimization according to the guidelines set out by the Human Proteome Organization (HuPO) for proteomic sample preparation. This chapter represents a snapshot of a work in progress; we anticipate that additional work in the near future will complete the story.

\subsection{Introduction}

Clinical proteomics promises the identification of protein biomarkers for the early diagnosis and prognosis of diseases. Since its inception in the mid-1990's, concerns regarding variability associated with pre-analytical variables (i.e., collection, transport, sample processing) were noted $^{115,170}$ and a strong need for standardization was recognized. In response to this need a variety of tools, including microfluidic technologies, have been developed for standardized sample preparation and processing for proteomic analysis.

Microfluidic systems are well suited for standardized biochemical assays, offering faster analysis times, superior analytical performance, reduced sample/reagent consumption, reduction of instrument footprint, the potential for portability, and automation. These advantages prompted the development of a wide range of microfluidic platforms specifically designed for proteomic sample processing steps, including extraction, resolubilization, reduction, alkylation, and digestion. There have been many inventive channel-based proteomic systems in which enzymes are immobilized in a section of the channel, such that the proteomic samples can be driven (electrokinetically or hydrodynamically) to the digestion site. ${ }^{37,171-173}$ Although such formats have favourable reaction kinetics because of the high surface-area-to-volume ratio and enzymeto-substrate ratio, such systems have typically been limited to single step processes, namely 
digestion. We are aware of two channel-based systems capable of carrying out a series of steps, including reduction, alkylation and digestion. These methods exploit the charges associated with proteins at particular pHs. The first method is called electrocapture, ${ }^{174}$ in which charged proteins are captured within a channel via electric fields, allowing for reagent to interact with the proteins while flowing through the channel. While this system is promising, the methodology involves significant operator-intervention, including switching valves and changing syringes in injection ports. The second method is known as the "proteomic reactor," ${ }^{47}$ in which digestive enzymes and proteomic samples are sequentially captured onto a strong cation exchange (SCX) resin as a function of $\mathrm{pH}$. A potential drawback of this system is the potential for proteins to irreproducibly desorb from the solid support during the constant flux of buffers and other solvents. Furthermore, while both electrocapture and the proteomic reactor facilitate the essential solution phase proteomic sample processing steps (reduction, alkylation, and digestion), it is difficult to imagine how these techniques could be combined with sample extraction, resolubilization, and sample cleanup.

Here, we propose to use digital microfluidics (DMF) to carry out all of the steps involved in front-end multistep proteomic processing. DMF is a fluid-handling technique in which discrete droplets of samples and reagents are manipulated (i.e., dispensed from reservoirs, split, merged and mixed) on an open surface by applying a series of electrical potentials to an array of electrodes. ${ }^{122,123}$ DMF has previously been used to (1) extract, clean-up and resolubilize proteins from biological fluids, ${ }^{50}$ as well as to (2) carry out solution phase reduction, alkylation and digestion step (chapter 3$)^{49,52}$. Here, we report the integration of steps (1) and (2) onto a single platform, as an important step towards achieving reliable, multiplexed biomarker diagnostic assays. In the future, we plan to optimize this method for repeatable measurements according to the guidelines set out by the Human Proteome Organization (HuPO) for proteomic sample preparation. $^{115,170}$

\subsection{Methods and Materials}

\subsubsection{Reagents and Materials}

Human serum albumin (HSA), Immunoglobulin G (IgG), Transferrin, Immunoglobulin A (IgA), Haptoglobin, TPCK-treated trypsin from bovine pancreas, iodoacetamide, Tris-HCl, ammonium bicarbonate, Pluronic F127, sodium dodecyl sulfate (SDS), Fluorinert FC-40, acetonitrile (ACN), 
trichloroacetic acid (TCA) and formic acid (FA) were purchased from Sigma Chemical (Oakville, ON). Tris-(2-carboxyethyl)phosphine hydrochloride (TCEP) and E6638 EnzChek Protease Assay Kit were purchased from Life Technologies/Invitrogen (Burlington, ON). C18 Ziptip pipet tips were purchased from Millipore (Etobicoke, ON). All aqueous solutions were prepared using deionized (DI) water that had a resistivity of $18 \mathrm{M} \Omega \cdot \mathrm{cm}$ at $25^{\circ} \mathrm{C}$. Unless otherwise indicated, all protein and processing reagent solutions were prepared in working buffer (100 mM Tris-HCl, pH 7.8, 0.08\% w/v Pluronic F127) immediately prior to use. Parylene-C dimer was from Specialty Coating Systems (Indianapolis, IN) and Teflon-AF was from DuPont (Wilmington, DE). Parylene-C dimer was from Specialty Coating Systems (Indianapolis, IN) and Teflon-AF was from DuPont (Wilmington, DE).

\subsubsection{Device Fabrication and Operation}

Digital microfluidic devices were fabricated in the University of Toronto Emerging Communications Technology Institute (ECTI) cleanroom facility, using a transparent photomask printed at Pacific Arts and Design (Markham, ON). Digital microfluidic device bottom plates bearing patterned chromium electrodes were formed by photolithography and etching as described previously ${ }^{164}$ and were coated with $15 \mu \mathrm{m}$ of Parylene-C and $200 \mathrm{~nm}$ of Teflon-AF. Parylene-C was applied using a vapor deposition instrument (Specialty Coating Systems), and Teflon-AF was spin-coated (1\% wt/wt in Fluorinert FC-40, $1600 \mathrm{rpm}, 60 \mathrm{~s})$ followed by postbaking on a hot-plate $\left(160^{\circ} \mathrm{C}, 10 \mathrm{~min}\right)$. The polymer coatings were removed from contact pads by gentle scraping with a scalpel to facilitate electrical contact for droplet actuation. In addition to patterned DMF device bottom plates, unpatterned DMF device top plates formed from indium tin oxide (ITO) coated glass substrates (Delta Technologies Ltd, Stillwater, MN) were coated with Teflon-AF (200 nm, as above).

The device used here featured an array of 68 square actuation electrodes $(2 \times 2 \mathrm{~mm}$ ea. $)$ connected to 10 reservoir electrodes $(5 \times 5 \mathrm{~mm}$ ea.), with inter-electrode gaps of 50-100 $\mu \mathrm{m}$. Devices were assembled with an unpatterned ITO-glass top plate and a patterned bottom plate separated by a spacer formed from 2 pieces of double-sided tape (total spacer thickness $140 \mu \mathrm{m}$ ). Unit droplet and reservoir droplet volumes on these devices were $600 \mathrm{~nL}$ and $5 \mu \mathrm{L}$, respectively. To actuate droplets, driving potentials $\left(200-250 \mathrm{~V}_{\mathrm{pp}}\right)$ were generated by amplifying the output of a function generator (Agilent Technologies, Santa Clara, CA) operating at $18 \mathrm{kHz}$. Droplets were 
sandwiched between two plates and were actuated by applying driving potentials between the top electrode (ground) and sequential electrodes on the bottom plate via the exposed contact pads. Droplet actuation was monitored and recorded by a CCD camera mounted on a lens.

\subsubsection{Workflow of DMF-driven and conventional Proteomic Assay}

The proteomic processing work-flow was carried out in seven distinct steps as illustrated in Figure 5.1: (i) precipitation, (ii) supernatant removal, (iii) washes (2×), (iv) reconstitution, (v) reduction, (vi) alkylation, and (vii) digestion. A crude aqueous model of human serum was formed from a mixture of HSA $(30 \mathrm{mg} / \mathrm{mL}), \operatorname{IgG}(10 \mathrm{mg} / \mathrm{mL})$, transferrin $(1 \mathrm{mg} / \mathrm{mL}), \operatorname{IgA}(1$ $\mathrm{mg} / \mathrm{L})$, and haptoglobin $(0.3 \mathrm{mg} / \mathrm{L})$ in working buffer and was used to evaluate the reproducibility of the process (steps i-vii). For step (i), a $600 \mathrm{~nL}$ droplet of model human serum and a second $600 \mathrm{~nL}$ droplet of cold precipitant (20\% TCA in DI water) were dispensed by DMF from their respective reservoirs and merged on an electrode (for reference purposes, this electrode is called the "extraction electrode") and the combined droplet was allowed to incubate for 5 minutes with device positioned on an ice pack $\left(-20^{\circ} \mathrm{C}\right)$. In step (ii), the supernatant was dispensed to the waste reservoir, leaving the precipitate on the extraction electrode. In cases where the supernatant droplet was difficult to remove (i.e., if the droplet size became too small and did not overlap with proceeding electrode), step (ii) was negated and the process was continued with step (iii). In step (iii) the precipitate was then washed by dispensing and driving one $600 \mathrm{~nL}$ droplet of rinse solution (neat acetone, $-20^{\circ} \mathrm{C}$ ) across the extraction electrode (and over the precipitate) to the waste reservoir. The $-20^{\circ} \mathrm{C}$ temperature was maintained by placing the device on top of an ice pack. This process was then repeated again. In step (iv), a $600 \mathrm{~nL}$ droplet of reconstitution buffer $\left(0.5 \%\right.$ SDS in $\left.\mathrm{dH}_{2} \mathrm{O}\right)$ was dispensed and driven to the extraction electrode and incubated to dissolve the protein (5 - $10 \mathrm{~min}$, room temp). Once partially dissolved, 2 - $3600 \mathrm{~nL}$ droplets of aqueous $50 \mathrm{mM}$ ammonium bicarbonate $(\mathrm{pH}$ 8.5) were dispensed and added the dissolving protein droplet for further incubation $(30-45$ min, room temp) in a humidified chamber (a Petri dish partially filled with $\mathrm{dH}_{2} \mathrm{O}$ ) to limit evaporation. In step (v) a $600 \mathrm{~nL}$ droplet of reducing agent (TCEP, $5 \mathrm{mM}$ in working buffer) was dispensed and merged with the droplet containing resolubilized protein followed by incubation (60 min, room temp) in the humidified chamber. In step (vi), a $600 \mathrm{~nL}$ droplet of alkylating agent (iodoacetamide, $10 \mathrm{mM}$ in $\mathrm{dH}_{2} \mathrm{O}$ ) was dispensed and merged with droplet from step (v) and incubated (45 min, room temp) in the humidified chamber in the dark. In step (vii), a $600 \mathrm{~nL}$ 
droplet of trypsin $(4.2 \mathrm{mg} / \mathrm{mL}$ in working buffer) was dispensed and merged with the droplet from step (vi) and incubated in a humidified chamber overnight ( 18 hours). In step (viii) a 600 $\mathrm{nL}$ droplet of formic acid (FA) was dispensed and merged with the droplet from step (vii), such that the final FA concentration was $0.1 \%$. Finally, samples were purified using a C18 ZipTip (Millipore, Etobicoke, $\mathrm{ON}$ ) and then dried using $\mathrm{N}_{2}$ gas. All samples were stored at $-20^{\circ} \mathrm{C}$ until analysis. Prior to analysis samples, were dissolved in $20 \mu \mathrm{L}$ of $0.1 \%$ formic acid in HPLC grade water.

Macroscale sample processing was implemented by pipettes in microcentrifuge tubes, and mirrored the DMF method, with two exceptions. First, each aliquot of sample and reagent in the macroscale method was $5 \mu \mathrm{L}$. Second, a centrifuge (13,000 rpm, $2 \mathrm{~min})$, aspiration, and resuspension procedure was used in place of steps (ii) and (iii) in the DMF method (the procedure was repeated twice, as in the DMF method).

\subsubsection{Reversed-Phase Capillary LC-MS/MS Analysis}

Reconstituted peptides were analyzed using a Proxeon EASY-nLC autosampler and nanopump HPLC system (Proxeon, Odense, Denmark) coupled to a LTQ Orbitrap Velos mass spectrometer (Thermo Fisher Scientific, San Jose, CA) via a nanoelectrospray ion source (Proxeon) interface located in Prof. Andrew Emili's lab at the University of Toronto. The reversed-phase capillary column was prepared by slurry packing 3- $\mu \mathrm{m}$ Jupiter C18 bonded particles (Phenomenex, Torrence, CA) into a 65-cm-long, 75- $\mu \mathrm{m}$-inner diameter fused silica capillary (Polymicro Technologies, Phoenix, AZ). After loading $2.5 \mu \mathrm{g}$ of peptides onto the column, the mobile phase was held at $100 \%$ buffer A $(0.1 \%$ formic acid $)$ for $20 \mathrm{~min}$, followed by a linear gradient from 0 to $70 \%$ buffer B $(0.1 \%$ formic acid in $90 \%$ acetonitrile) over $85 \mathrm{~min}$. Each full MS scan $(\mathrm{m} / z$ 400-2000) was followed by collision-induced MS/MS spectra (normalized collision energy setting of $35 \%$ ) for the 10 most abundant ions. The dynamic exclusion duration was set to $1 \mathrm{~min}$, the heated capillary was maintained at $200^{\circ} \mathrm{C}$, and the ESI voltage was held at $2.2 \mathrm{kV}$.

\subsubsection{Data Analysis}

RAW files were extracted from the mass spectrometry data with the extractms program (http://fields.scripps.edu/sequest/extractms.html) and submitted for database searching using SEQUEST and a modified UniProt/SwissProt protein database FASTA file. Search parameters 
were set to allow for one missed cleavage site, one variable modification of +16 for Methionine oxidation and one fixed modification of +57 for Cysteine carbamidomethylation using precursor ion tolerances of $3 \mathrm{~m} / \mathrm{z}$, before search and $20 \mathrm{ppm}$ after search. Protein hits were filtered using the StatQuest program with a confidence level of $99 \%$. The abundance of the proteins is calculated based on spectral counting, which is the number of the spectra matched to the peptides from this protein

\subsection{Results and Discussion}

Analysis of the serum proteome represents a significant challenge due to the nature of sample, which is characterized by: (1) large dynamic range, (2) low abundance of target analytes, and (3) high level of salts and other interfering compounds. In proof-of-principle experiments described here, a model system was used, comprising a mixture of HSA, IgG, transferrin, IgA, and Haptoglobin. These five proteins represent the most abundant proteins found in human serum and their concentrations were prepared at their average levels in serum. In the initial work reported here, this mixture was analyzed "as-is"; in the future, we propose to add target proteins at low concentrations to evaluate the suitability of this method for reproducible biomarker detection.

\subsubsection{Analysis of model serum on DMF device}

The array geometry on DMF devices is well suited for sequential biochemical assays, including proteomic sample processes (chapter 3). ${ }^{49,50}$ As depicted in Figure 5.1, a DMF device was developed to control a diverse set of proteomic samples and reagents. The essential steps for proteomic analysis, including protein extraction, resolubilization, reduction, alkylation and enzymatic digestion were all carried out on a single platform.

Protein extraction and resolubilization is an essential proteomic step used to isolate the component of interest (proteins) and remove/reduce unwanted matrix that may interfere with analysis. As shown, in a typical DMF analysis, a droplet containing model serum was dispensed (Figure 5.1-1) and merged with a droplet of precipitant (20\% TCA). TCA is commonly used for protein precipitation, as it causes proteins to unfold leading to hydrophobic aggregration. ${ }^{175,176}$ This process was carried out on an ice pack $\left(-20^{\circ} \mathrm{C}\right)$ to reduce the solubility of the proteins. After proteins were fully precipitated, the supernatant was actuated away to the waste reservoir. In 
some cases, the addition of a rinse droplet (acetone, $-20^{\circ} \mathrm{C}$ ) was required to assist in the removal (Figure 5.1-2 to 5.1-3). Cold acetone was used as the rinse solution to solubilize any remaining non-protein consituents and to minimize the resolubilization of proteins. Protein reconstitution was carried out using a mixture of SDS and ammonium bicarbonate (Figure 5.1-4). SDS is often used to resolubilize the most hydrophobic proteins (e.g., membrane proteins), ${ }^{177}$ but the final SDS concentration must be reduced to ensure that it does not compromise MS analysis. Ammonium bicarbonate is also a solubilization agent ${ }^{178}$ and was used to decrease the concentration of SDS used. It should be noted that the extraction protocol used here was initially based on the first publication on DMF-driven protein extraction. ${ }^{50}$ In the intial work, the authors used 70/30 v/v chloroform/ACN and 1\% SDS in $10 \mathrm{mM}$ borate as their rinse and resolubilization buffer, respectively, which is different from the current protocol. Modifications to this initial protocol were made to successfully transition the extraction step to the downstream protein processing steps. Using a fluorogenic assay, the authors of the preliminary work ${ }^{50}$ demonstrated recovery levels similar to those typically found in macro-scale systems using centrifuges (> 80\% recovery) and in the future a similar assay will be used to assess the quantitative recovery of the protocol used here.

After resolubilizing the proteins, solution-phase biochemical processing was implemented. A droplet containing the resolubilized protein sample was merged with a droplet of reducing agent (TCEP) (Figure 5.1-5). The stability, selectivity, and rapid reaction rate at room temperature makes TCEP a good replacement for the more commonly used dithiothreitol (DTT), which must be removed prior to further processing. ${ }^{129}$ After reduction, the sample droplet was merged with a droplet containing iodoacetamide (Figure 5.1-6), which covalently binds to cysteine groups preventing reformation of disulfide bonds. Finally, the sample droplet was merged with a droplet containing trypsin to digest the protein into constitutive peptides (Figure 5.1-7). These seven steps address the essential elements of front-end proteomic processing. 


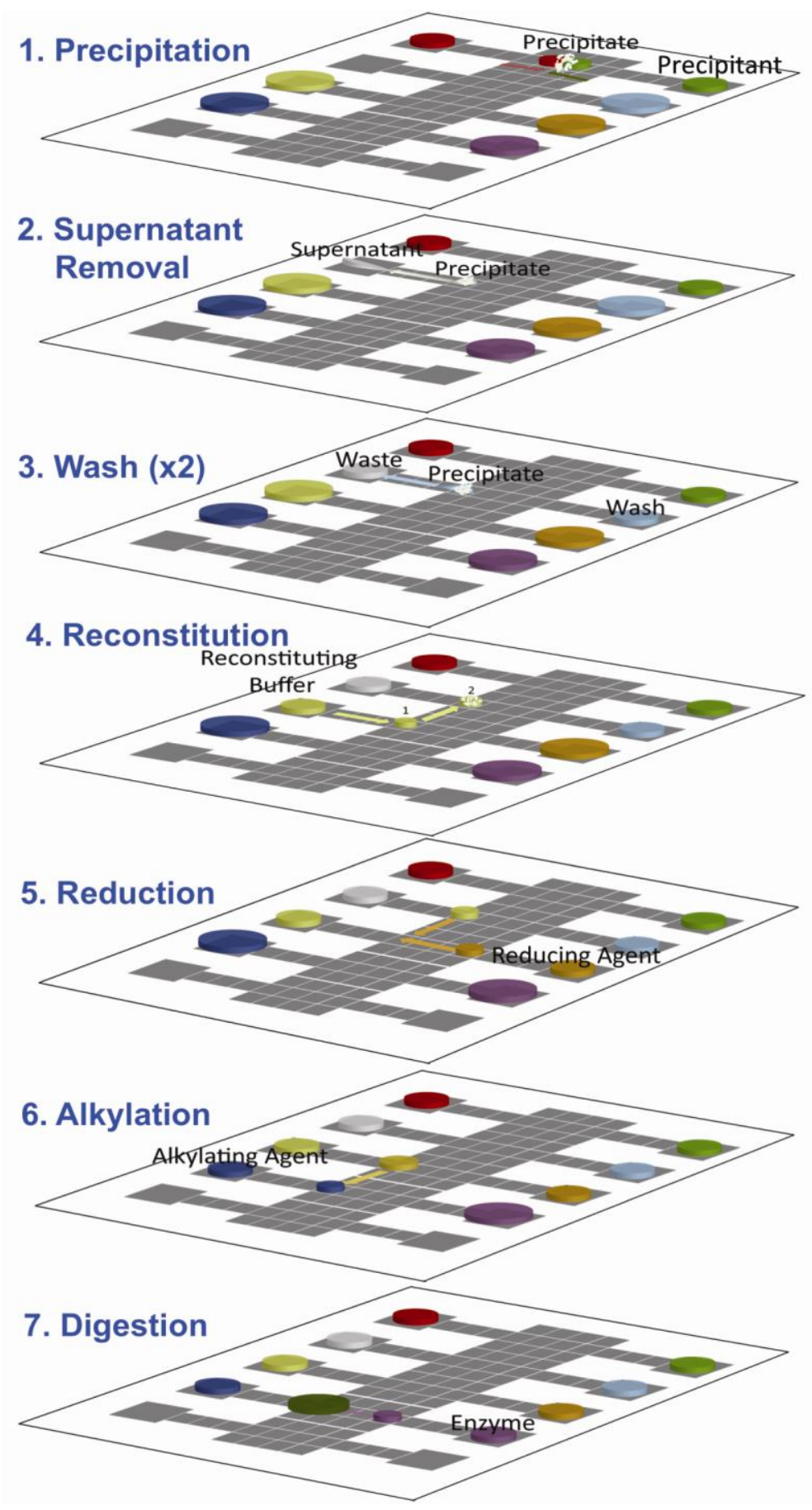

Figure 5.1: Sequence of schematics depicting a typical proteomic sample processing workup. (1) Droplets containing model human serum (total protein concentration $42.3 \mathrm{mg} / \mathrm{L}$ ) and precipitant $(20 \% \mathrm{TCA}$ ) are dispensed from reservoirs and merged on an ice pack. After 5 minutes (2) the supernatant is actuated to the waste reservoir. (3) Precipitant is washed twice consecutively by a droplet of cold rinse solution (acetone). (4) The precipitant is dissolved in the reconstitution buffer $(0.5 \% \mathrm{SDS} / 50 \mathrm{mM}$ ammonioum bicarbonate). Once the protein pellet is resolubilized (5) a droplet of TCEP $(5 \mathrm{mM})$ is dispensed from a reservoir, merged and actively mixed with the protein sample droplet. After incubating, (6) a droplet of iodoacetamide $(10 \mathrm{mM})$ is dispensed and merged with the sample droplet and actively mixed. After incubating again, (7) the sample droplet is merged with a droplet of trypsin $(1: 10 \mathrm{w} / \mathrm{w}$ enzyme to protein ratio) and then incubated a final time. 


\subsubsection{Analysis of model serum on DMF device macroscale processing}

Spectral counting ${ }^{179}$ was used as a metric to compare the reproducibility of DMF-driven proteomic sample processing to its macroscale equivalent. Spectral counting is a label-free method in which the abundance of a particular protein is correlated to the number of MS/MS spectra obtained for that protein in a proteomic analysis. Figure 5.2 shows the spectral counts obtained for each protein, with samples processed by DMF (blue) or in the macroscale (i.e., pipettes and centrifuge tubes; red). A $t$-test demonstrates there is no significant difference between the data generated by the two methods $(\mathrm{P}>0.05)$. The coefficients of variation of the measurements $(43,24,42,12$, and $22 \%$ for the DMF method, and 50, 42, 68, 11, and 39\% for the macroscale method, for HSA, IgG, transferrin, IgA, and Haptoglobin, respectively) are similar, indicating that the methods had similar levels of precision. Thus, our hypothesis that the DMF method would prove to be more reproducible was not confirmed in this initial data set. In future work, we may find that implementation of automated systems with impedance sensing and feedback control ${ }^{180}$ may reduce the variance in the DMF system. Regardless, the methods reported here represent the first fully automated DMF system for protein sample preparation, and I propose that the parameters described here represent a launching point for future studies in this area. 


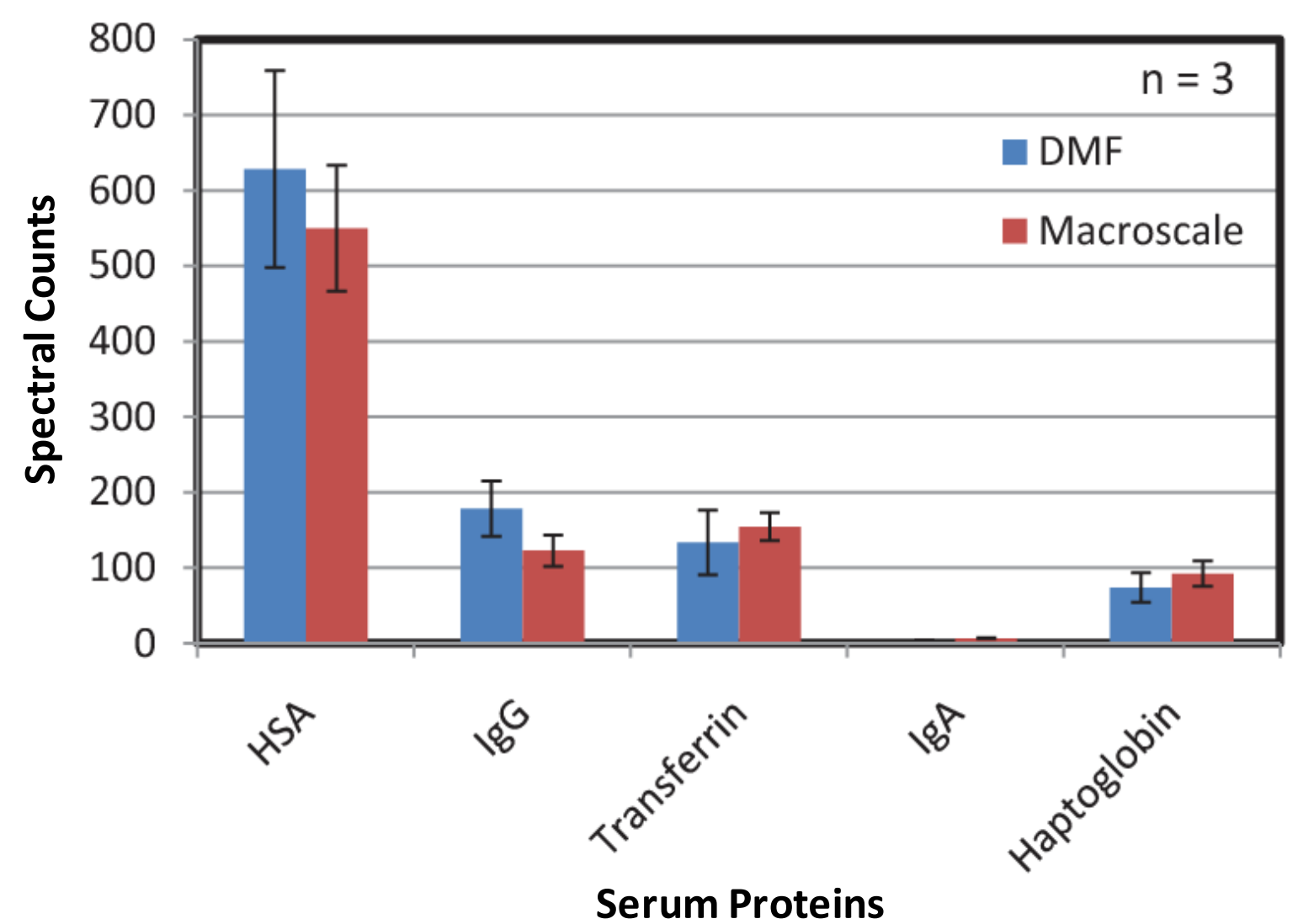

Figure 5.2: Spectral counts of proteins in model serum (BSA, IgG, Transferrin, IgA, Haptoglobin) after processing by DMF vs. conventional front-end proteomic procedures. All samples were analyzed in triplicate.

\subsection{Conclusion}

The lack of standardized sample handling and processing in proteomics is a major limitation for the field. Here, I have shown that digital microfluidics (DMF) can implement a complex, multiple-step proteomic sample preparation protocol starting from crude model serum. This section demonstrates initial work towards a fully integrated platform for proteome profiling. 


\section{Concluding Remarks and Future Work}

Proteome profiling has great promise in the field of medicine and has been touted as a useful predictive tool that can aid in the diagnosis and prognosis of potential diseases. There are a number of technological hurdles that have limited the impact of proteomics. In my thesis, I describe a series of new techniques and methods that may someday help overcome these hurdles. Here, I summarize my contributions in this area and propose suggestions for future work.

Making DMF technologically compatible with biochemical assays - dealing with non-specific adsorption (Chapter 2)

Chapter 2 presents a method to prevent non-specific adsorption of biomolecules onto DMF devices. The method uses low concentrations of pluronics as a solution additive. This technique significantly limits the amount of biofouling facilitating the movement/translation of protein droplets on DMF at 1000-fold higher concentrations relative to samples without the additive. Pluronic additive transforms DMF into a platform feasible for biochemical assays, by allowing a wider range and higher concentration of proteins and reagents to be handled. Although pluronics as a solution additive appears to have little effect on the proteomic processes described in this thesis (chapters 2 and 3) ${ }^{49,128}$, many downstream steps (e.g., separation or detection methods) require the removal of pluronics. I propose that in the future, it would be useful to develop techniques to either remove pluronics from droplets in-line in DMF devices (for example, by integrating with solid-phase extraction ${ }^{181}$ ), or to develop device coatings bearing immobilized pluronics or other anti-fouling agents.

\section{Multi-step proteomic sample handling and processing on DMF (Chapters 3 and 5)}

Chapter 3 presents a new method for proteomic sample processing using an integrated microfluidic device, in an attempt to address the variances introduced by non-standardized manual sample handing and processing. This chapter demonstrates the compatibility of DMF with proteomic solutions and reagents, the ability to carry out multi-step proteomic processes (i.e., reduction, alkylation, and digestion), and the capacity to evaluate each reaction step and its associated variance. This work demonstrated that DMF is a robust tool capable of implementing the key steps in proteomic processing. 
Chapter 5 presents proof-of-principle work towards a fully integrated proteomic sample processing platform and represents a continuation of the methods described in chapter 3 . In this work, six common processing steps in proteomics, including protein extraction, sample clean-up, resolubilization, reduction, alkylation, and enzymatic digestion were implemented by DMF. Spectral counting was used to evaluate overall reproducibility of DMF-driven proteomics versus its macroscale equivalent. The initial data set does not support the hypothesis that the DMF method is more reproducible than the macro-scale technique; however, the dispensing method used in the DMF method was relatively crude, relying on manual application of potentials to the driving electrodes. Upon implementation of an impedance-based feedback control system, I propose that the precision of droplet dispensing/metering will increase. ${ }^{134,135}$ For these methods to fulfill their potential in becoming robust and reliable, additional innovation in electronics and microfabrication is required. Moreover, the initial data set presented in Chapter 5 represents the beginning (rather than the end) of a long story. Methods should be developed to assess and optimize (1) the extraction efficiency, (2) the range of protein concentrations compatible with the methods, (3) the ability to identify low-concentration target biomarkers, and (4) whether these methods are applicable to the analysis of real serum samples. Scientists who work on these problems in the future may find my initial methods a useful starting point for their (substantial and challenging) work.

\section{Functional proteomic components on DMF (Chapter 4)}

Chapter 4 presented a new strategy for proteolytic digestion on DMF to achieve cleaner spectra for greater confidence in protein characterization. This method overcomes the limitations inherent in homogenous (solution-phase) digestion (e.g., the digestion methods described in chapters 3 and 5), such as the need to maintain low enzyme:substrate ratio (which results in lengthy incubation times and may require elevated temperatures), autolysis (i.e., self-digestion of enzyme), and digestion artifacts (e.g., transpeptidation, deamidation/oxidation). These complications were avoided by immobilizing enzymes on hydrogel discs, which acts as a 3-D scaffold for the enzymes. DMF-driven proteomic assays employing this technique (heterogeneous digestion) generated spectra with better quality and increased sequence coverage compared to solution-phase digestion. Moreover, because digestion with a single enzyme is often not sufficient to identify proteins of interest in complex samples, I developed methods for parallel digestion, in which a proteomic sample is split into two aliquots and each is exposed to a 
different gel bearing a different enzyme (i.e., trypsin, pepsin) for digestion. This technique increases sequence coverage and confidence in identification, which illustrates the potential for parallel proteomic sample processing for DMF paired with gel discs digestion.

The literature suggests that enzymes immobilized on hydrogel supports are significantly more thermally stable compared to solution-phase digestion. Although the technique described in chapter 4 generated higher quality results, temperature was not a parameter that was investigated. The stability of enzymes immobilized in hydrogel discs at elevated temperatures merits investigation. I speculate that elevated temperatures would further increase the throughput of the proteomic process by increasing the kinetics of the digestion step. Enzymes immobilized on hydrogel disc coupled with DMF prove to be a useful new functional component towards miniaturization and automation in proteomic sample processing when combined with the proofof-concept methods described in chapter 5 . 


\section{Appendix 1 - Hydrogel Discs for Digital Microfluidics}

Hydrogels are networks of hydrophilic polymer chains that are swollen with water. Hydrogels are useful for a wide range of applications because their pores serve as stable sites for immobilizing proteins and cells. We report here the marriage of hydrogels with digital microfluidic devices. Until recently, digital microfluidics, a fluid handling technique in which discrete droplets are controlled electrostatically on the surface of an array of electrodes, has been used only for homogeneous systems involving liquid reagents. Here, we demonstrate for the first time that cylindrical hydrogel discs can be incorporated into digital microfluidic systems and that these discs can be systematically addressed by droplets of reagents. Droplet movement is observed to be unimpeded by interaction with the gel discs, and gel discs remain stationary when droplets pass through them. Analyte transport into gel discs was observed to be identical to diffusion in cases in which droplets are incubated with gels passively, but transport was enhanced when droplets were continually actuated through the gels. The system was demonstrated to be useful for generating integrated enzymatic microreactors and for threedimensional cell culture. This paper demonstrates a new combination of techniques for lab-on-achip systems which we propose will be useful for a wide range of applications.

\section{A1.1 Introduction}

Hydrogels are hydrophilic polymers with an open network structure that are swollen with water. ${ }^{182-185}$ Hydrogels are uniquely useful for a wide range of applications because they can serve as stable niches for the immobilization of proteins and cells. ${ }^{186}$ For the former case, protein-bearing hydrogels are often formed to serve as microreactors for use in enzymatic digestion, ${ }^{165}$ catalysis, ${ }^{187}$ and biosensors. ${ }^{188}$ For the latter case, hydrogels are often used as scaffolds for tissue engineering in two-dimensional ${ }^{189-192}$ (2D) and three-dimensional ${ }^{186,193,194}$ (3D) formats. In addition to being useful for encapsulating proteins and cells, hydrogels are useful for sensing operations involving temperature, ${ }^{195} \mathrm{pH},{ }^{196}$ and quantitation of analytes, ${ }^{196}$ and can be used in a wide range of applications including drug delivery, ${ }^{197,198}$ cosmetics, ${ }^{199,200}$ and food. $^{201-203}$

There has been great enthusiasm for combining hydrogels with microchannel-based lab-on-achip systems. ${ }^{204-212}$ For example, microfluidic systems are useful for reagent delivery and 
collection of products from hydrogel-based enzyme microreactors. ${ }^{204}$ Likewise, microfluidic systems for culturing cells encapsulated in hydrogel structures are useful because fluidic ports are easily incorporated for nutrient delivery and waste removal. ${ }^{207}$ These advances are promising and useful; however, the plumbing complexities incurred with controlling many different reagents simultaneously make the combination of microchannels and hydrogels an imperfect match for all applications. Moreover, in continuous flow microchannel systems, analytes generated by enzymes or cells immobilized in hydrogels can diffuse away from the gels, complicating the analysis.

Digital microfluidics (DMF) is an alternative to microchannels for controlling fluids in miniaturized devices. In DMF, discrete droplets are controlled electrostatically by applying a series of electrical potentials to an open array of electrodes (with no channels or walls) covered by a hydrophobic insulator. ${ }^{53,54,122}$ DMF enables facile control over many different reagents for multi-step processes, a property that has been useful for enzyme assays ${ }^{72,73,75,206}$ and for applications involving cells. ${ }^{71,213-219}$ In these initial applications, enzymatic bioreactors and cell culture and assays were implemented in homogeneous aqueous droplets manipulated by DMF. We propose that the marriage of DMF with hydrogels represents a welcome combination for forming miniaturized enzyme bioreactors and 3D cell culture scaffolds.

We report here the first integration of hydrogel structures onto digital microfluidic (DMF) devices for applications involving encapsulated proteins and cells. A review paper published in $2007^{220}$ included a short description of a procedure to mix two reagents to form a hydrogel in a DMF device. But beyond this paper, we are not aware of any other reports of combining hydrogels with DMF. Thus, this paper is the first to report digital microfluidic applications of hydrogels including reagent exchange, enzyme microreactors, and 3D cell culture. We propose that the combination of digital microfluidics with hydrogels will be useful for a wide range of applications. 


\section{A1.2 Methods and Materials}

\section{A1.2.1 Reagents and Material}

Agarose (two forms: low-melting point and ultra-low gelling temperature) and all other reagents (unless noted otherwise) were from Sigma Aldrich Canada (Oakville, ON) and were used as received. Cell culture reagents were from Invitrogen/Life Technologies (Burlington, ON), and Pluronic P105 was generously donated by BASF Brenntag Canada (Toronto, ON). Parylene-C dimer was from Specialty Coating Systems (Indianapolis, IN), and Teflon-AF was purchased from DuPont (Wilmington, DE). Deionized (DI) water had a resistivity of $18 \mathrm{M} \Omega \bullet \mathrm{cm}$ at $25^{\circ} \mathrm{C}$.

\section{A1.2.2 DMF Device Fabrication and Operation}

Digital microfluidic devices were fabricated in the University of Toronto Emerging Communications Technology Institute (ECTI) cleanroom facility, using a transparent photomask printed at Pacific Arts and Design (Markham, ON). Digital microfluidic device bottom plates bearing patterned chromium electrodes were formed by photolithography and etching as described previously, ${ }^{72,221}$ and were coated with $15 \mu \mathrm{m}$ of Parylene-C and $200 \mathrm{~nm}$ of TeflonAF. Parylene-C was applied using a vapor deposition instrument (Specialty Coating Systems), and Teflon-AF was spin-coated (1\% wt/wt in Fluorinert FC-40, 1600 rpm, $60 \mathrm{~s}$ ) followed by post-baking on a hot-plate $\left(160^{\circ} \mathrm{C}, 10 \mathrm{~min}\right)$. The polymer coatings were removed from contact pads by gentle scraping with a scalpel to facilitate electrical contact for droplet actuation. In addition to patterned DMF device bottom plates, unpatterned glass microscope slides and DMF device top plates formed from indium tin oxide (ITO) coated glass substrates (Delta Technologies Ltd, Stillwater, MN) were coated with Teflon-AF (200 nm, as above).

The device used here featured an array of 68 square actuation electrodes ( 2 x $2 \mathrm{~mm}$ ea.) connected to 10 reservoir electrodes ( 5 x $5 \mathrm{~mm}$ ea.), with inter-electrode gaps of 50-100 $\mu \mathrm{m}$. Devices used for imaging cells incorporated 0.8 x $0.8 \mathrm{~mm}$ transparent windows (i.e., regions with no chromium) at the corners of some electrodes to assist with imaging. ${ }^{215}$ Devices were assembled with an unpatterned ITO-glass top plate and a patterned bottom plate separated by a spacer formed from 2 pieces of double-sided tape (total spacer thickness $140 \mu \mathrm{m}$ ). To actuate droplets, driving potentials $\left(200-250 \mathrm{~V}_{\mathrm{pp}}\right)$ were generated by amplifying the output of a function generator (Agilent Technologies, Santa Clara, CA) operating at $18 \mathrm{kHz}$. Droplets were sandwiched between two plates and were actuated by applying driving potentials between the top 
electrode (ground) and sequential electrodes on the bottom plate via the exposed contact pads. Droplet actuation was monitored and recorded by a CCD camera mounted on a lens.

\section{A1.2.3 Formation of Gel Discs}

Agarose was dissolved in DI water to a concentration of $0.5-4.0 \mathrm{wt} \%$ by incubating the mixture in an oven at $70^{\circ} \mathrm{C}$ for $1 \mathrm{~h}$. Low-melting point agarose was used for experiments not involving cells, and ultra-low gelling temperature agarose was used for experiments involving cells. Some mixtures included food coloring dyes or $10 \mu \mathrm{m}$ diameter polystyrene beads (Sigma Aldrich Canada) to make the gels easier to visualize. After removing the mixture from the oven, $0.4-20$ $\mu \mathrm{L}$ aliquots were pipetted and sandwiched between two unpatterned Teflon AF-coated glass slides held together with 2 pieces of double-sided tape as a $140 \mu \mathrm{m}$ spacer. These assemblies were placed on a cold pack to allow the droplets to gel $(2 \mathrm{~min})$. The resulting gel discs were $\sim 1$ to $5 \mathrm{~mm}$ in diameter and $140 \mu \mathrm{m}$ tall and could be retrieved from the glass-slide assembly using tweezers. Figure A1.1 shows a picture of gel discs and droplets of water, which demonstrates that the gel disc geometry is fixed in a cylindrical shape (in comparison to the water droplets, which are domed). For use with digital microfluidics, gel discs were sandwiched between a bottom plate and a top plate, with each disc positioned such that it straddled the interface between two electrodes on the bottom plate.

\section{A1.2.4 Gel Disc Dispensing Measurements}

In each experiment, a $2.5 \mu \mathrm{L}$ DI water droplet was dispensed from a reservoir and then merged onto a $2 \mathrm{~mm}$ dia. $4 \mathrm{wt} \%$ agarose gel disc. A sub-droplet was then dispensed away from the gel disc. Pictures were collected throughout the process, and volumes were estimated using Image J software. The amount of water that remained on a gel after dispensing was calculated and averaged for 10 droplets on 10 different gels.

\section{A1.2.5 Gel Disc Solution Exchange Assay}

In each experiment, a $5 \mu \mathrm{L}$ droplet of $10 \mu \mathrm{M}$ fluorescein in $100 \mu \mathrm{M}$ borate buffer ( $\mathrm{pH}$ 9.2) was dispensed from a reservoir and merged with a $2 \mathrm{~mm}$ dia. $4 \mathrm{wt} \%$ agarose gel disc for passive or active exchange. For passive exchange, no further action was taken. For active exchange, the droplet was repeatedly actuated through the gel at $\sim 2.4 \mathrm{~mm} / \mathrm{s}$ by alternately charging the two electrodes on either side of the disc. Pictures of the gel were collected using a microscope 
equipped with a fluorescent imaging module for 480/40 nm (Leica DM2000, Illinois, USA) every 5 (passive) or 2.5 (active) minutes. Each condition was repeated three times on three different gel discs.

The images were analyzed to measure the fluorescence in the gel using Image $\mathbf{J}$ software. Briefly, in each image, the fluorescence intensity of the area of the inner $250 \mu \mathrm{m}$ radius of the gel was divided by the average intensity of the same area outside the gel (in the droplet-at-large) to compensate for photobleaching. These corrected data were then plotted as a function of incubation time then normalized to the value of the last time point in each graph. Lines were fit to this data to determine the inflection point, correlating with saturation of the gel with fluorescein. The diffusion coefficient (passive) and apparent transport rate (active) were calculated as the square of the radius of the gel over the time it took to saturate the gel.

\section{A1.2.6 Enzyme Microreactor Assay}

Enzymes were covalently attached to agarose gel discs using methods similar to those developed by Guisan and coworkers ${ }^{165,210,211}$ to prime the gels for attachment; $2 \mathrm{~mm}$ dia. 4 wt \% agarose gel discs were isolated on Teflon AF-coated glass slides. A 3.5 $\mu \mathrm{L}$ aliquot of DI water was pipetted onto each gel, followed by $1 \mu \mathrm{L}$ of $1.7 \mathrm{~N} \mathrm{NaOH}$ containing $68 \mu \mathrm{g}$ of $\mathrm{NaBH}_{4}$. The slide was placed on a cold pack and $0.7 \mu \mathrm{L}$ of glycidol was pipetted onto the gel. After 5 minutes, the gel was removed from the cold pack and placed in a humidifier for $18 \mathrm{~h}$ at $25^{\circ} \mathrm{C}$. After incubation the gel was washed 5 times with $10 \mu \mathrm{L}$ of DI water. The gel was then oxidized by adding $30 \mu \mathrm{L}$ of DI water and $6 \mu \mathrm{L}$ of $2 \mathrm{M}$ aqueous $\mathrm{NaIO}_{4}$ and then incubated in a humidifier for $2 \mathrm{~h}$ at $25^{\circ} \mathrm{C}$. Finally, the gel was washed 5 times with $10 \mu \mathrm{L}$ aliquots of DI water.

After priming, gel discs were conjugated with alkaline phosphatase. A working buffer was formed from sodium borate in DI water (0.1 M, pH 10.0). $20 \mu \mathrm{L}$ of $0.05 \mathrm{mg} / \mathrm{mL}$ alkaline phosphatase in working buffer was pipetted onto each primed gel, which was then incubated in a humidifier for $72 \mathrm{~h}$ at $25^{\circ} \mathrm{C}$. The gel was washed 5 times with $10 \mu \mathrm{L}$ aliquots of working buffer and then stabilized by adding $20 \mu \mathrm{L}$ of working buffer containing $20 \mu \mathrm{g} \mathrm{NaBH}_{4}$ and incubating for 30 minutes at $25^{\circ} \mathrm{C}$. Finally each gel was washed 5 times with $10 \mu \mathrm{L}$ aliquots of sodium phosphate buffer (25 mM, pH 7.0). 
For heterogeneous enzyme assays, a reaction buffer was formed from $10 \mathrm{mM}$ diethanolamine and $1 \mathrm{mM} \mathrm{MgCl}_{2}$ in DI water $(0.1 \mathrm{M}, \mathrm{pH} \mathrm{10.0)}$. Gels functionalized as described above were positioned into DMF devices and conditioned by dispensing $10 \mu \mathrm{L}$ droplets of reaction buffer from reservoirs and merging them onto the gels. A $2.5 \mu \mathrm{L}$ droplet of fluorescein diphosphate $(0$ to $40 \mu \mathrm{g} / \mathrm{mL}$ ) in reaction buffer was then dispensed and merged onto each conditioned gel and incubated for 3 minutes. Droplets were then dispensed from the gel and $2 \mu \mathrm{L}$ of each dispensed droplet was collected and mixed with $18 \mu \mathrm{L}$ DI water in a well in a 384-well plate. Fluorescence was measured using a PheraStar multiwell plate reader with $\lambda_{\mathrm{ex}}=485 \mathrm{~nm}$; $\lambda_{\mathrm{em}}=520 \mathrm{~nm}$; focal height $=9.0 \mathrm{~mm}$; gain $=1$ ). Each concentration of fluorescein diphosphate was evaluated three times on three different gels.

\section{A1.2.7 3D Cell Culture}

Complete cell culture medium was formed from DMEM (Sigma-Aldrich) supplemented with $10 \%$ fetal bovine serum and 1\% penicillin-streptomycin. NIH 3T3 cells were grown to near confluency in complete medium in T-25 flasks in an incubator at $37^{\circ} \mathrm{C}$ with $5 \% \mathrm{CO}_{2}$. At the beginning of each experiment, cells were detached using a solution of trypsin $(0.25 \% \mathrm{w} / \mathrm{v})$ and EDTA $(1 \mathrm{mM})$ for 5 minutes and then centrifuged at $173 \mathrm{x} \mathrm{g}$ for 5 minutes. The supernatant was removed and the cells were resuspended in complete medium at $10^{7}$ cell/mL.

To prepare cell-laden gel discs, 4 wt\% ultra-low melting agarose in DI water was diluted with complete cell media to a final concentration of agarose ranging from 0.4 to $3.6 \mathrm{wt} \%$. The $10^{7}$ cell/mL suspension in medium was added to these agarose solutions to obtain a suspension containing $10^{6}$ cell $/ \mathrm{mL}$. The results of initial experiments indicated that $0.58 \mathrm{wt} \%$ agarose in such suspensions was an appropriate concentration for 3D cell growth (see results and discussion section), and this concentration was used for all subsequent experiments. Droplets of this agarose/cell mixture were pipetted onto DMF bottom plates (straddling an electrode bearing a transparent window, see the fabrication section), covered with top plates and then placed in a humidified chamber at $4^{\circ} \mathrm{C}$ for 15 minutes to gel the discs. Devices were stored in an incubator at $37^{\circ} \mathrm{C} / 5 \% \mathrm{CO}_{2}$ for 1 day, and gels were then visualized through the transparent windows using a Leica DM2000 microscope (Leica Microsystems Canada, Richmond Hill, ON).

In some cases, cells in gel discs were viability stained or fixed and stained for nuclei and F-actin. All steps were implemented by digital microfluidics -- droplets of reagents were dispensed from 
reservoirs, merged onto gels, incubated, and then dispensed away from them. For the former (viability staining), a $2 \mu \mathrm{L}$ droplet of $1 \mu \mathrm{M}$ calcein-AM (Invitrogen/Life Technologies) in PBS supplemented with $0.05 \%$ Pluronic F68 was delivered to each gel and incubated for 30 minutes at room temperature. Each gel was then washed twice with $2 \mu \mathrm{L}$ droplets of PBS, after which cells were imaged by fluorescence microscopy (Leica DM2000). For the latter (cell fixation and staining), gel discs were washed with $2 \mu \mathrm{L}$ droplets of PBS and then exposed to $2 \mu \mathrm{L}$ droplets of $3.7 \%$ (w/v) paraformaldehyde in PBS supplemented with $0.05 \%$ Pluronic F68 for 15 minutes (chapter 2). ${ }^{128}$ The discs were then washed three times with $2 \mu \mathrm{L}$ droplets of PBS and dehydrated by incubation in $2 \mu \mathrm{L}$ droplets of cold acetone for 10 minutes at $-20{ }^{\circ} \mathrm{C}$. The discs were then rehydrated by exposure to $2 \mu \mathrm{L}$ droplets of PBS for 10 minutes before incubation with $2 \mu \mathrm{L}$ droplets of dye solution containing $50 \mu \mathrm{g} / \mathrm{mL}$ phalloidin, $1 \mu \mathrm{g} / \mathrm{mL}$ DAPI and $0.02 \%$ (w/v) Pluronic P105 in PBS for 30 minutes at room temperature. ${ }^{59}$ The discs were then washed three times with $2 \mu \mathrm{L}$ droplets of PBS and the DMF top plate was replaced with a \#1 coverslip for confocal imaging on a Leica TCS SP2 (Leica Microsystems).

\section{A1.3 Results and Discussion}

\section{A1.3.1 Design and Behaviour of Microgel Discs for Digital Microfluidics}

A previous report ${ }^{220}$ described a method for the preparation of gels on DMF devices. In that initial work, droplets of alginate were combined with droplets of calcium chloride and the resulting mixture was observed to be non-movable (and thus was assumed to be gelled). In this

initial work, ${ }^{220}$ it was not clear from the brief discussion whether the gels were subsequently addressable by liquid droplets for subsequent applications. Here, we introduce the use of thermoreversible agarose polymers on DMF devices, and demonstrate the use of these gels for use with enzyme microreactors and 3D cell culture. We used two forms of agarose, low-melting point agarose which melts at $65^{\circ} \mathrm{C}$ and gels at $30^{\circ} \mathrm{C}$, and ultra-low gelling temperature agarose which melts at $\leq 50^{\circ} \mathrm{C}$ and gels between $8-17^{\circ} \mathrm{C}$. Figure A1.1 shows the morphology of a gel disc formed using this technique -- when formed sandwiched between two plates, the gels maintain their shapes, and can be used and re-used in different devices and experiments. In most of the work reported here, we used $\sim 2 \mathrm{~mm}$-diameter discs to match the dimensions of the DMF actuation electrodes used here, but in practice, any desirable size can be formed by adjusting the volume of the agarose mixture prior to gelling. 


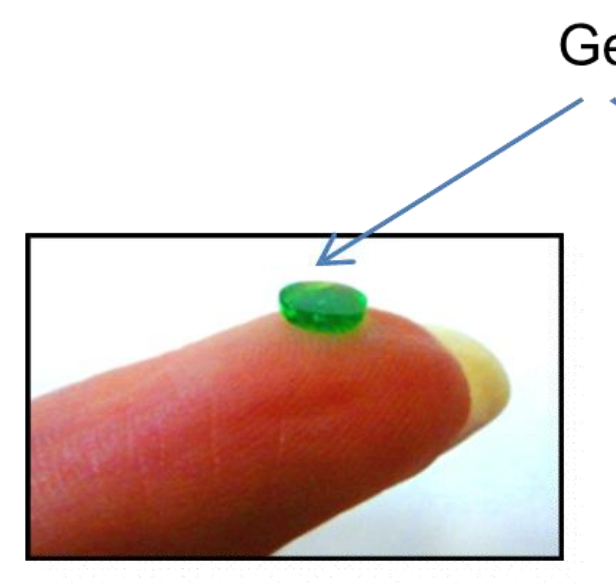

Finger
Gel

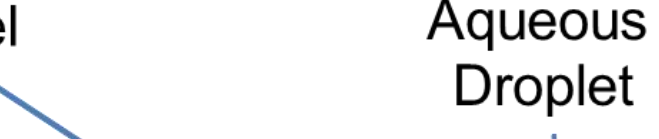

Figure A1.1: Images of gel discs on a finger (left) and on a digital microfluidic device with no top plate (right). Aqueous droplets (yellow) are included in the image on the right to demonstrate the differences in geometry (i.e., the gel discs are cylinders, and the droplets have a domed shape). The gels and droplets contain dye to make them visible.

A first question in our experiments was whether the agarose gel discs would remain stationary or would become mobile when exposed to moving droplets on DMF devices. We hypothesized that adhesion and friction would keep the gel discs stationary, and this turned out to be the case. In fact, over the course of hundreds of experiments, gel discs were never observed to move, which is similar to our observations of porous polymer monoliths on DMF devices. ${ }^{181}$

A second question about gel discs on digital microfluidic devices was whether water droplets could be dispensed from the gels, or whether the water would remain permanently bound to the extremely hydrophilic gel discs. In fact, droplet dispensing was not only possible but quite straightforward. As shown in Figure A1.2, when the three electrodes adjacent to a gel disc are actuated in succession, a droplet (connected by a tail) is pulled away from the gel until it pinches off. This occurs reproducibly and predictably. After dispensing, a small amount of the original droplet volume remains on the gel. To characterize this quantitatively, ten $2.5 \mu \mathrm{L}$ droplets of water were merged and dispensed from hydrogel discs, and on average, $~ 10 \%$ of the volume $(178 \pm 13 \mathrm{~nL})$ remains bound to the gel, and $\sim 90 \%$ is dispensed. 


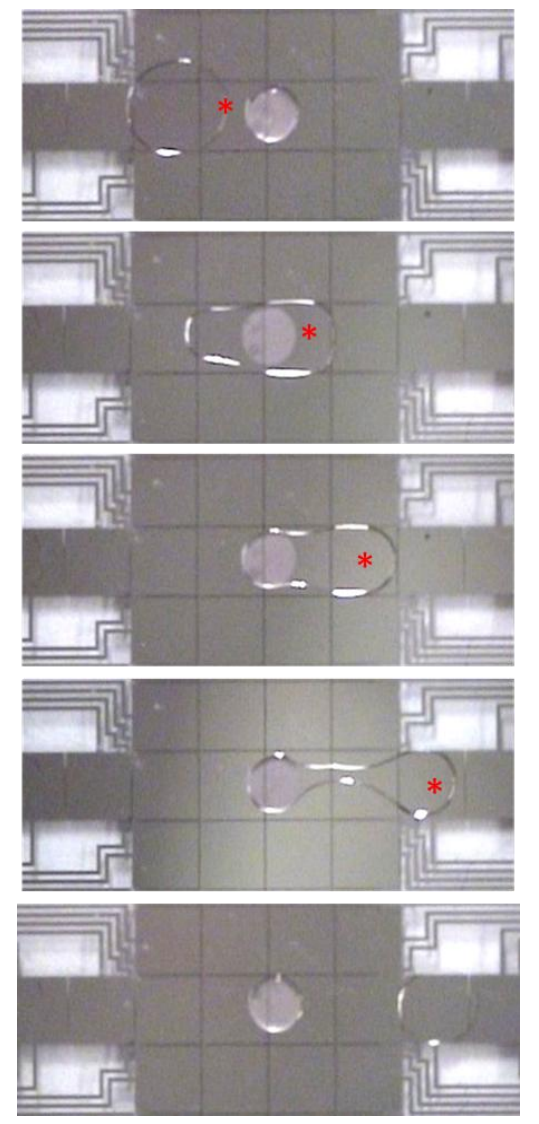

Figure A1.2: Droplet actuation and hydrogel discs in digital microfluidics. A series of images from a movie (topto-bottom) depicting the merging of a $2 \mu \mathrm{L}$ droplet with an immobilized gel disc, followed by dispensing of a portion of the volume. The red asterisks $\left(^{*}\right)$ denote which electrode was activated in each frame. The gel disc contains a suspension of $2 \mu \mathrm{m}$ dia. beads to make it visible.

A third question about the techniques described here was the nature and rate of analyte exchange between gel disc and droplet during droplet movement. To address this question, droplets containing fluorescein were merged with gels and were incubated either passively (with no further droplet actuation) or actively (with constant droplet actuation through the gel) as described in the methods section. The fluorescence intensities measured in the gel discs were plotted as a function of time. As shown in Figure A1.3, the trends for solution exchange with the gel disc have a similar profile for both active and passive cases; the fluorescence intensity increases linearly until it saturates and is constant thereafter. The inflection point between the linear increase and the saturation state represents the time required for fluorescein molecules to completely fill the gel. For passive exchange this process required $28.8 \pm 1.5 \mathrm{~min}$, while active 
exchange was $\sim 3 \mathrm{x}$ faster, at $8.7 \pm 0.6 \mathrm{~min}$. From this, we estimate the diffusion coefficient for the passive case to be $0.58 \times 10^{-9} \mathrm{~m}^{2} / \mathrm{s}$ and the apparent rate for active transport to be $1.9 \times 10^{-9}$ $\mathrm{m}^{2} / \mathrm{s}$. The measured diffusion coefficient for fluorescein in the gel disc is very similar to the known diffusion coefficient for fluorescein in water, $0.49 \times 10^{-9} \mathrm{~m}^{2} / \mathrm{s} .{ }^{222}$ This is expected, as the $4 \%$ agarose used here has large pores $\left(\sim 243 \mathrm{~nm}\right.$ for $4 \mathrm{wt} \%$ agarose $\left.{ }^{223}\right)$ which should not impede the movement of small molecules like fluorescein. The faster exchange observed for the active case suggests that the electrostatic droplet actuation facilitates convection into the gel.

The observations of gel disc behaviour in digital microfluidic systems reported above are interesting, and they suggest a host of future studies into the effects (both for 2D transport, as shown in Figure A1.3, and for 3D transport, not evaluated here) of gel size, droplet volume, pore size, molecule size, and actuation velocity. But the observations recorded here demonstrate proof of principle for the combination of digital microfluidics with hydrogels, and were sufficient to inspire exploration of the combination of digital microfluidics and gel discs to applications involving immobilized enzymes and cells, described below. 
(a)

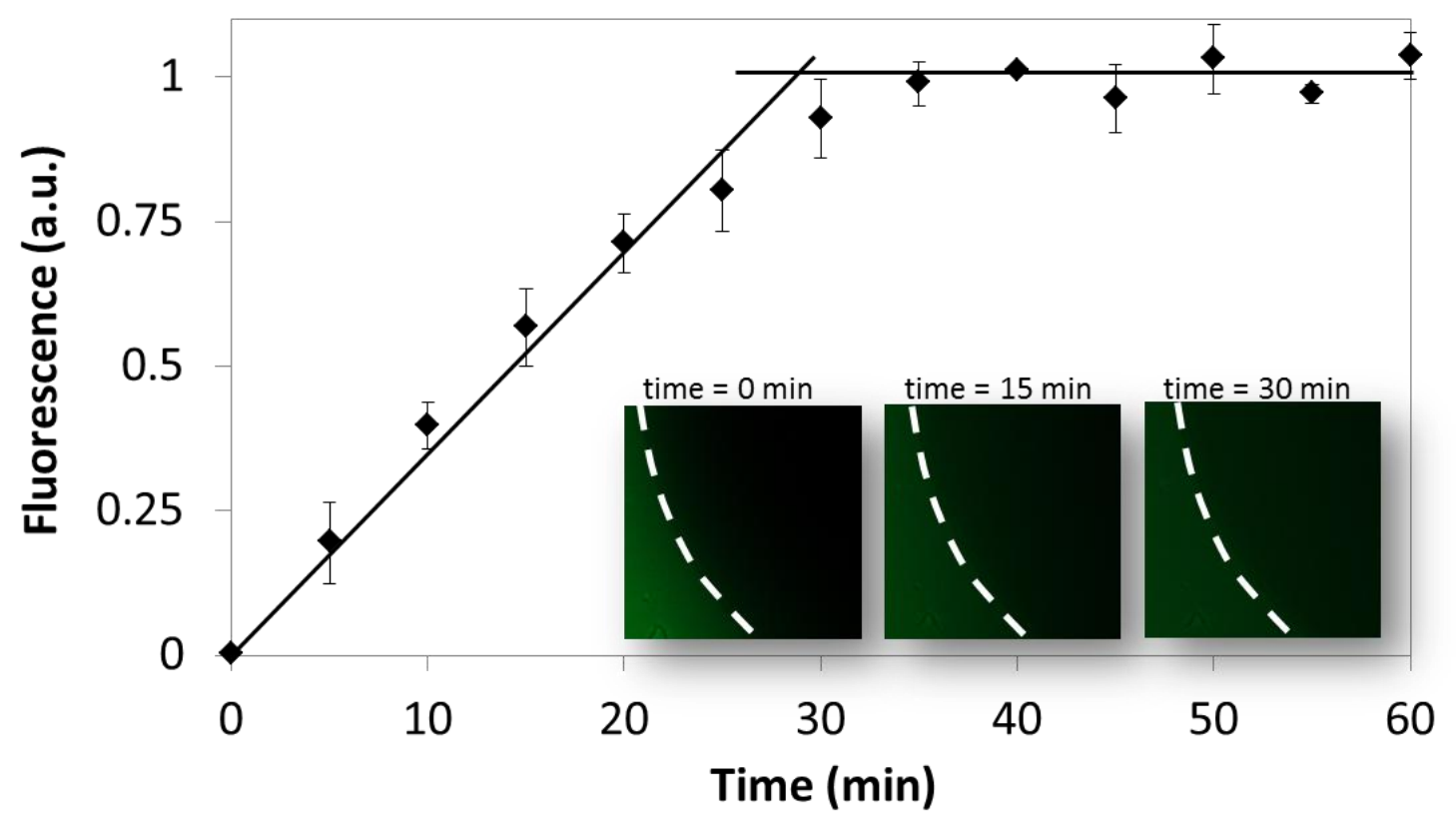

(b)

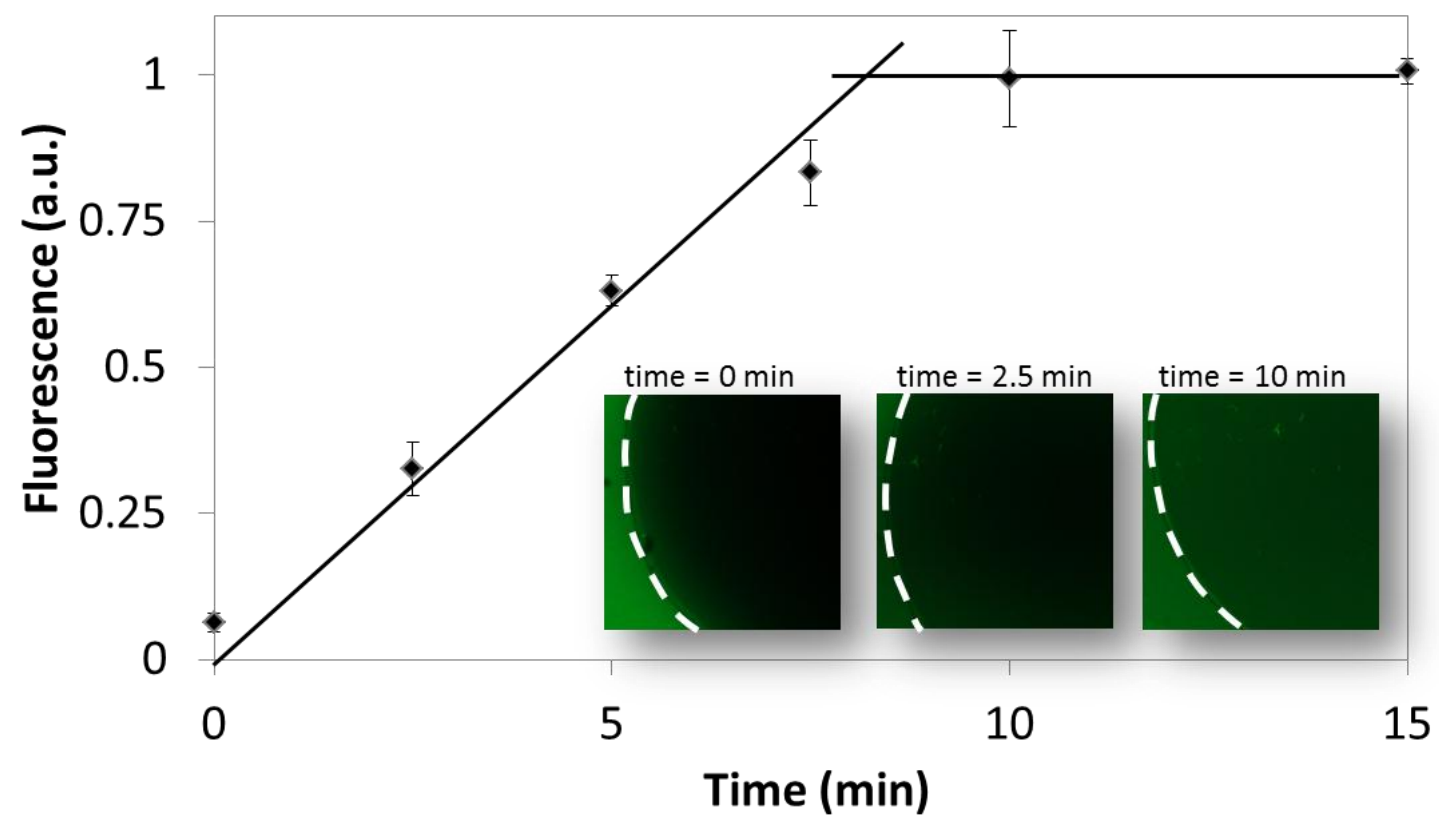

Figure A1.3: Transport assay. Droplets containing fluorescein were merged with gel discs and allowed to incubate either passively (a) or actively (b) and fluorescence in the gel was plotted as a function of time. The insets are representative fluorescent images of gels at different times (the dashed white lines indicate the edge of the discs). In both the passive and active cases, the fluorescence increases linearly inside the gel until saturation. The inflection point between the linear increase and saturation regions represents the time required for fluorescein to permeate through the whole gel. This time is $\sim 3 \mathrm{x}$ less for active transport relative to passive transport. The experiments were replicated 3 times on three different gels and error bars are \pm 1 S.D. 


\section{A1.3.2 Application \#1: Enzyme Microreactor}

As a first demonstration of an application for digital microfluidics and hydrogel discs, we explored the implementation of enzyme microreactors. Enzyme microreactors are often used to perform small-scale enzymatic digestions of small amounts of rare protein samples or for multiplexed screening of many protein samples. For such applications, is advantageous to immobilize the enzyme onto a substrate in a bio-compatible niche to preserve functionality. ${ }^{204}$ We report here a method for immobilizing enzymes to agarose gel discs by oxidizing alcohol groups to aldehydes, and then coupling gel the aldehyde groups to protein amine groups. Here, we report the first hydrogel enzyme microreactor implemented by digital microfluidics. Gel discs covalently functionalized with alkaline phosphatase served as microreactors. Droplets containing fluorescein diphosphate (which is non-fluorescent) were dispensed and merged onto the gels to allow for the enzyme to cleave off the phosphate groups, generating the fluorescent product, fluorescein. After incubating, the droplets were dispensed and their fluorescence was measured. Figure A1.4 shows the fluorescence of the dispensed droplets as a function of fluorescein diphosphate concentration. As expected, the fluorescence increased linearly with substrate concentration.

The digital microfluidic enzymatic microreactor described here has a number of differences relative to those formed in conventional enclosed microchannel systems. ${ }^{204,207,210}$ For example, microchannel systems are well suited for very low $(\sim \mathrm{nL})$ sample volumes. This property makes microchannel enzyme microreactors useful for analytical applications, but such systems are often not easily adapted for use for preparative applications. In contrast, the digital microfluidic system described here is appropriate for larger $(\sim \mu \mathrm{L})$ sample volumes, with straightforward means of recovery of products for preparative applications. In addition, in the digital microfluidic system, the reagents and products are isolated in an immobile droplet (surrounded by air), such that products cannot diffuse away. We propose that the two types of systems are complementary, with application dictating the appropriate format. 


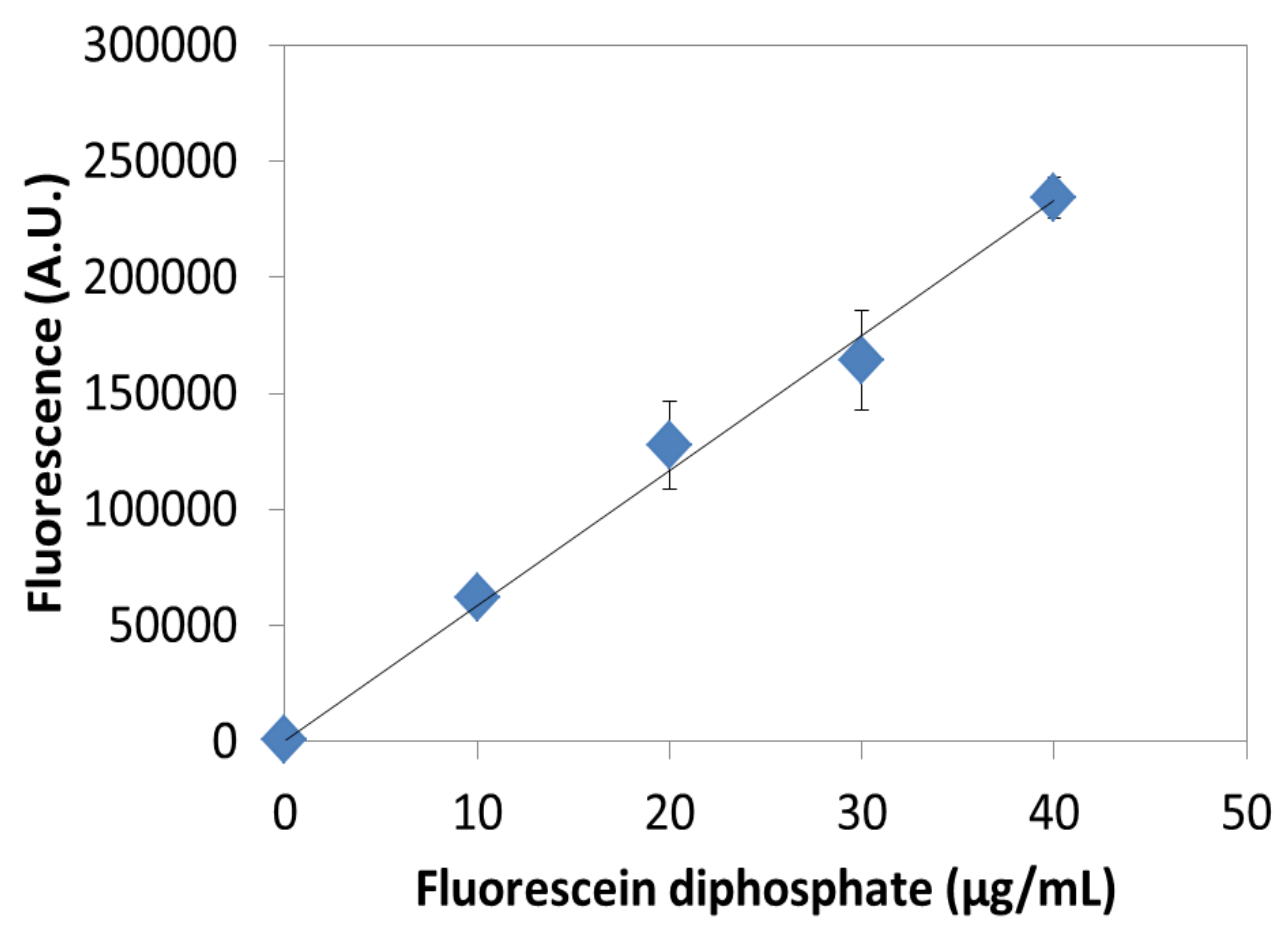

Figure A1.4: Enzyme Microreactor. Gel discs were formed with covalently linked alkaline phosphatase. Droplets containing fluorescein diphosphate were merged onto the discs and after incubation, the fluorescence of dispensed droplets was reported as a function of the concentration of fluorescein diphosphate. The experiments were replicated 3 times and error bars are \pm 1 S.D. A least squares line of best fit through the data has an $\mathrm{R}^{2}=0.9929$.

\section{A1.3.3 Application \#2: 3D Cell Culture}

As a second demonstration of an application for digital microfluidics and hydrogel discs, we explored the use of this system for 3D cell culture. NIH-3T3 fibroblasts were seeded in a solution of ultra-low melting temperature agarose, which was then gelled into discs and incorporated into DMF devices. The ability of the cells to form 3D networks was found to be highly dependent on agarose concentration, which is consistent with previous reports. ${ }^{65}$ Gel discs with agarose concentrations greater than $1 \mathrm{wt} \%$ resulted in cells with (undesirable) spherical morphologies, and discs with agarose densities lower than $0.45 \mathrm{wt} \%$ had morphologies that were similar to those found in conventional two-dimensional systems (not shown). It is likely that high-concentration agarose gels tightly constrain the cells, while low-concentration agarose solutions failed to fully transition into gels. Regardless, as demonstrated in Figure A1.5, 0.58 wt $\%$ agarose gel was a useful concentration for forming 3D networks of cells. 
As shown in Figure A1.5a, fibroblasts cultured in the digital microfluidic/hydrogel disc format exhibited stellate bipolar morphologies consistent with culture in high-modulus extracellular matrix $^{224}$ and formed 3D networks of interconnected cells. This morphology is different than what is observed in cells grown in traditional 2D systems (such cells have flat and unconnected morphologies, not shown). As shown in Figure A1.5b, cells embedded in gel discs remained viable as determined by staining with calcein AM, and high cell viability was observed for up to 7 days, after which experiments were discontinued. Cells were also fixed and stained for nuclei and F-actin in situ using digital microfluidic actuation. This process (described in detail in the experimental section) underscores the robustness of the new methods reported here -- gels were sequentially exposed to seven sets of droplets (as depicted in Figure A1.2) to rinse, fix, rinse, dehydrate, rinse, stain, and rinse the cells, respectively.

As shown in Figure A1.5c, cells embedded in $0.58 \mathrm{wt} \%$ agarose discs self-organized into tightly packed networks with cell-cell interaction (clustering). The dense packing of cells is a key feature of the engineering of tissues and is known to affect cell phenotype by creating molecular gradients and affecting matrix stiffness. ${ }^{225}$ In addition to the tight packing of cells, the shift from cell-substrate interactions typical of two-dimensional cell culture to cell-cell interactions is likely to change cell behaviour as a result of increased cellular communication. ${ }^{226}$ Given the widespread interest in the development of 3D cell culture systems as a better model of in vivo phenotypes $^{227}$ (relative to traditional 2D culture systems), we propose that the techniques reported here, in which $3 \mathrm{D}$ scaffolds can be independently and sequentially addressed with reagents in an automated, miniaturized format, will be useful for numerous applications in tissue engineering. 

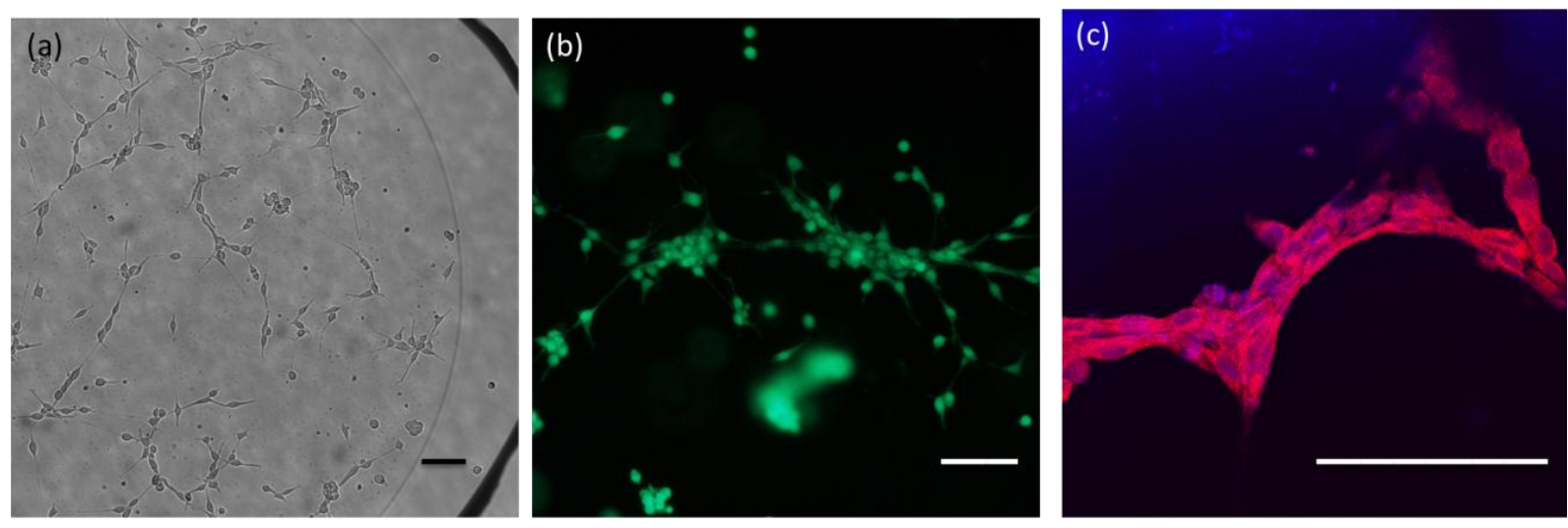

Figure A1.5: Three-Dimensional Cell Culture. $3 \mathrm{~T} 3$ cells were cultured and imaged in $0.58 \mathrm{wt} \%$ agarose gel discs on DMF devices. Cells were imaged through transparent windows on DMF devices using bright field (a), fluorescence (b) with calcein AM dye and confocal fluorescence (c) with DAPI (shown in red) and phalloidin (shown in blue) microscopies. The scale bar in each image is $25 \mu \mathrm{m}$.

\section{A1.4 Conclusion}

We have demonstrated that cylindrical hydrogel discs can be incorporated in digital microfluidic devices, and that droplets of reagents can be systematically addressed to them. The gel discs can be functionalized with proteins to act as an enzymatic microreactor, as demonstrated in the reaction of alkaline phosphatase with fluorescein diphosphate. The gel discs can also be used for 3D cell culture on DMF devices, as demonstrated by the behaviour of NIH-3T3 cells grown in such systems, which formed tightly packed networks rife with cell-cell connections. We propose that combining hydrogels with DMF will be useful for many applications in the future. 


\section{References}

1. Wilkins, M. Proteomics data mining. Expert Review of Proteomics 6, 599-603 (2009).

2. Wilkins, M.R. et al. Progress with proteome projects: why all proteins expressed by a genome should be identified and how to do it. Biotechnology \& Genetic Engineering Reviews 13, 19-50 (1996).

3. 38997 Entries Found in PubMed Search on Feb 5, 2012 with Parameters: Keyword (as Written) “'Proteomic”,' Publication Year “'2002-2012'”, Document Type “'Journal”,.

4. Blackstock, W.P. \& Weir, M.P. Proteomics: quantitative and physical mapping of cellular proteins. Trends in Biotechnology 17, 121-127 (1999).

5. Monti, M., Orrù, S., Pagnozzi, D. \& Pucci, P. Functional proteomics. Clinica Chimica Acta 357, 140-50 (2005).

6. Sung, H.-J. \& Cho, J.-Y. Biomarkers for the lung cancer diagnosis and their advances in proteomics. BMB reports 41, 615-25 (2008).

7. Li, M. et al. Proteomic analysis of serum in lung cancer induced by 3-methylcholanthrene. Journal of Biomedicine \& Biotechnology 2009, 397910 (2009).

8. Lopez, M.F. et al. A novel, high-throughput workflow for discovery and identification of serum carrier protein-bound peptide biomarker candidates in ovarian cancer samples. Clinical Chemistry 53, 1067-74 (2007).

9. Pang, J. et al. Profiling Protein Markers Associated with Lymph Node Metastasis in Prostate Cancer by DIGE-based Proteomics Analysis. Journal of Proteome Research 9, 216-226 (2010).

10. Kim, H.-jung et al. Identification of S100A8 and S100A9 as Serological Markers for Colorectal Cancer. Journal of Proteome Research 8, 1368-1379 (2009). 
11. Sun, Y. et al. Differential proteomics identification of HSP90 as potential serum biomarker in hepatocellular carcinoma by two-dimensional electrophoresis and mass spectrometry. International Journal of Molecular Sciences 11, 1423-33 (2010).

12. Ray, S. et al. Proteomic technologies for the identification of disease biomarkers in serum: advances and challenges ahead. Proteomics 11, 2139-61 (2011).

13. Washburn, M.P., Wolters, D. \& Yates, J.R. Large-scale analysis of the yeast proteome by multidimensional protein identification technology. Nature Biotechnology 19, 242-7 (2001).

14. Fränzel, B. \& Wolters, D. a Advanced MudPIT as a next step toward high proteome coverage. Proteomics 3651-3656 (2011).doi:10.1002/pmic.201100056

15. Tran, J.C. et al. Mapping intact protein isoforms in discovery mode using top-down proteomics. Nature 480, 254-8 (2011).

16. Cui, W., Rohrs, H.W. \& Gross, M.L. Top-down mass spectrometry: Recent developments, applications and perspectives. The Analyst 136, 3854-64 (2011).

17. Siuti, N. \& Kelleher, N.L. Decoding protein modifications using top-down mass spectrometry. Nature Methods 4, 817-821 (2007).

18. Scigelova, M. \& Makarov, A. Orbitrap mass analyzer--overview and applications in proteomics. Proteomics 6 Suppl 2, 16-21 (2006).

19. Michalski, A. et al. Ultra high resolution linear ion trap Orbitrap mass spectrometer (Orbitrap Elite) facilitates top down LC MS/MS and versatile peptide fragmentation modes. Molecular \& Cellular Proteomics (2011).doi:10.1074/mcp.O111.013698

20. Li, Z. et al. Systematic Comparison of Label-Free, Metabolic Labeling, and Isobaric Chemical Labeling for Quantitative Proteomics on LTQ Orbitrap Velos. Journal of Proteome Research (2012).doi:10.1021/pr200748h 
21. Nagaraj, N. et al. Systems-wide perturbation analysis with near complete coverage of the yeast proteome by single-shot UHPLC runs on a bench-top Orbitrap. Molecular \& Cellular Proteomics 11, (2012).

22. Anderson, N.L. The Human Plasma Proteome: History, Character, and Diagnostic Prospects. Molecular \& Cellular Proteomics 1, 845-867 (2002).

23. Yeo, L.Y., Chang, H.-C., Chan, P.P.Y. \& Friend, J.R. Microfluidic Devices for Bioapplications. Small 7, 12-48 (2010).

24. Weibel, D.B. \& Whitesides, G.M. Applications of microfluidics in chemical biology. Current opinion in chemical biology 10, 584-91 (2006).

25. Harrison, D.J., Manz, A., Fan, Z., Ludi, H. \& Widmer, H.M. Capillary electrophoresis and sample injection systems integrated on a planar glass chip. Analytical Chemistry 64, 1926-1932 (1992).

26. Manz, A. et al. Planar chips technology for miniaturization and integration of separation techniques into monitoring systems - Capillary electrophoresis on a chip. Journal of Chromatography A 593, 253-258 (1992).

27. Harrison, D.J. et al. Micromachining a miniaturized capillary electrophoresis-based chemical analysis system on a chip. Science 261, 895-7 (1993).

28. Jeon, N.L. et al. Generation of Solution and Surface Gradients Using Microfluidic Systems. Langmuir 16, 8311-8316 (2000).

29. Dertinger, S.K.W., Chiu, D.T., Jeon, N.L. \& Whitesides, G.M. Generation of Gradients Having Complex Shapes Using Microfluidic Networks. Analytical Chemistry 73, 12401246 (2001).

30. Hong, J.W. \& Quake, S.R. Integrated nanoliter systems. Nature Biotechnology 21, 117983 (2003).

31. Zimmermann, M., Bentley, S., Schmid, H., Hunziker, P. \& Delamarche, E. Continuous flow in open microfluidics using controlled evaporation. Lab on a Chip 5, 1355-9 (2005). 
32. Dahlin, A.P., Bergstro, S.K., Andre, P.E., Markides, K.E. \& Bergquist, J. Poly(dimethylsiloxane)-Based Microchip for Electrophoresis with an Integrated Electrospray Emitter Tip. Analytical Chemistry 77, 5356-5363 (2005).

33. Long, Z., Shen, Z., Wu, D., Qin, J. \& Lin, B. Integrated multilayer microfluidic device with a nanoporous membrane interconnect for online coupling of solid-phase extraction to microchip electrophoresis. Lab on a Chip 7, 1819-24 (2007).

34. Yue, G.E. et al. Protein digestion and phosphopeptide enrichment on a glass microchip. Analytica Chimica Acta 564, 116-22 (2006).

35. Mao, X. et al. Integrated lectin affinity microfluidic chip for glycoform separation. Analytical Chemistry 76, 6941-7 (2004).

36. Hardt, S. \& Hahn, T. Microfluidics with aqueous two-phase systems. Lab on a chip 434442 (2011).doi:10.1039/c1lc20569b

37. Wang, C. et al. Integration of immobilized trypsin bead beds for protein digestion within a microfluidic chip incorporating capillary electrophoresis separations and an electrospray mass spectrometry interface. Rapid Communications in Mass Spectrometry 14, 1377-83 (2000).

38. Jin, L.J., Ferrance, J., Sanders, J.C. \& Landers, J.P. A microchip-based proteolytic digestion system driven by electroosmotic pumping. Lab on a chip 3, 11-8 (2003).

39. Liuni, P., Rob, T. \& Wilson, D.J. A microfluidic reactor for rapid, low-pressure proteolysis with on-chip electrospray ionization. Rapid Communications in Mass Spectrometry 24, 315-320 (2010).

40. Kim, Y.D., Park, C.B. \& Clark, D.S. Stable sol-gel microstructured and microfluidic networks for protein patterning. Biotechnology and Bioengineering 73, 331-7 (2001).

41. Sakai-Kato, K., Kato, M. \& Toyo'oka, T. Creation of an on-chip enzyme reactor by encapsulating trypsin in sol-gel on a plastic microchip. Analytical Chemistry 75, 388-93 (2003). 
42. Huang, Y. et al. Zeolite nanoparticle modified microchip reactor for efficient protein digestion. Lab on a Chip 6, 534-9 (2006).

43. Kato, M., Inuzuka, K., Sakai-kato, K. \& Toyo, T. Monolithic Bioreactor Immobilizing Trypsin for High-Throughput Analysis. Analytical Chemistry 77, 1813-1818 (2005).

44. Palm, A.K. \& Novotny, M.V. Analytical characterization of a facile porous polymer monolithic trypsin microreactor enabling peptide mass mapping using mass spectrometry. Rapid Communications in Mass Spectrometry 18, 1374-82 (2004).

45. Carlier, J. et al. Integrated microfabricated systems including a purification module and an on-chip nano electrospray ionization interface for biological analysis. Journal of Chromatography A 1071, 213-222 (2005).

46. Gao, J., Xu, J., Locascio, L.E. \& Lee, C.S. Integrated Microfluidic System Enabling Protein Digestion, Peptide Separation, and Protein Identification. Analytical chemistry 73, 2648-2655 (2001).

47. Ethier, M., Hou, W., Duewel, H.S. \& Figeys, D. The proteomic reactor: a microfluidic device for processing minute amounts of protein prior to mass spectrometry analysis. Journal of Proteome Research 5, 2754-9 (2006).

48. Unger, M.A., Chou, H.-pu, Thorsen, T., Scherer, A. \& Quake, S.R. Monolithic Microfabricared Valves and Pumps by Multilayer Soft Lithography. Science 288, 113-116 (2000).

49. Luk, V.N. \& Wheeler, A.R. A digital microfluidic approach to proteomic sample processing. Analytical Chemistry 81, 4524-30 (2009).

50. Jebrail, M.J. \& Wheeler, A.R. Digital microfluidic method for protein extraction by precipitation. Analytical chemistry 81, 330-5 (2009).

51. Nelson, W.C. et al. Incubated Protein Reduction and Digestion on an Electrowetting-onDielectric Digital Microfluidic Chip for MALDI-MS. Analytical Chemistry 82, 9932-9937 (2010). 
52. Chatterjee, D., Ytterberg, A.J., Son, S.U., Loo, J.A. \& Garrell, R.L. Integration of protein processing steps on a droplet microfluidics platform for MALDI-MS analysis. Analytical Chemistry 82, 2095-101 (2010).

53. Pollack, M.G., Fair, R.B. \& Shenderov, A.D. Electrowetting-based actuation of liquid droplets for microfluidic applications. Applied Physics Letters 77, 1725 (2000).

54. Lee, J., Moon, H., Fowler, J., Schoellhammer, T. \& Kim, C.-J. Electrowetting and electrowetting-on-dielectric for microscale liquid handling. Sensors and Actuators A: Physical 95, 259-268 (2002).

55. Moon, H., Cho, S.K., Garrell, R.L. \& Kim, C.-J. Low voltage electrowetting-on-dielectric. Journal of Applied Physics 92, 4080 (2002).

56. Berthier, J. et al. Actuation potentials and capillary forces in electrowetting based microsystems. Sensors and Actuators A: Physical 134, 471-479 (2007).

57. Chatterjee, D., Hetayothin, B., Wheeler, A.R., King, D.J. \& Garrell, R.L. Droplet-based microfluidics with nonaqueous solvents and solutions. Lab on a Chip 6, 199-206 (2006).

58. Shapiro, B., Moon, H., Garrell, R.L. \& Kim, C.-J. Equilibrium behavior of sessile drops under surface tension, applied external fields, and material variations. Journal of Applied Physics 93, 5794-5811 (2003).

59. Quilliet, C. \& Berge, B. Electrowetting: a recent outbreak. Current Opinion in Colloid \& Interface Science 34-39 (2001).

60. Berge, B. Electrocapillarity and wetting of insulator films by water. C. R. Acad. Sci. Paris, Ser. II. 317, 157-163 (1993).

61. Jones, T.B. On the Relationship of Dielectrophoresis and. Langmuir 18, 4437-4443 (2002).

62. Jones, Thomas B., Fowler, J.D., Chang, Y.S. \& Kim, C.-jin Frequency-Based Relationship of Electrowetting and Dielectrophoretic Liquid Microactuation. Langmuir 7646-7651 (2003). 
63. Jones, T.B., Wang, K.L. \& Yao, D.J. Frequency-dependent electromechanics of aqueous liquids: electrowetting and dielectrophoresis. Langmuir 20, 2813-2818 (2004).

64. Chatterjee, D., Shepherd, H. \& Garrell, R.L. Electromechanical model for actuating liquids in a two-plate droplet microfluidic device. Lab on a Chip 9, 1219-29 (2009).

65. Jones, T.B., Gunji, M., Washizu, M. \& Feldman, M.J. Dielectrophoretic liquid actuation and nanodroplet formation. Journal of Applied Physics 89, 1441 (2001).

66. Wheeler, A.R., Moon, H., Kim, C.-J., Loo, J.A. \& Garrell, R.L. Electrowetting-based microfluidics for analysis of peptides and proteins by matrix-assisted laser desorption/ionization mass spectrometry. Analytical chemistry 76, 4833-8 (2004).

67. Wheeler, A.R. et al. Digital microfluidics with in-line sample purification for proteomics analyses with MALDI-MS. Analytical Chemistry 77, 534-40 (2005).

68. Moon, H., Wheeler, A.R., Garrell, R.L., Loo, J. a \& Kim, C.-J.C. An integrated digital microfluidic chip for multiplexed proteomic sample preparation and analysis by MALDIMS. Lab on a chip 6, 1213-9 (2006).

69. Velev, O.D., Prevo, B.G. \& Bhatt, K.H. On-chip manipulation of free droplets. Nature 426, 515-6 (2003).

70. Mukhopadhyay, R. Diving into droplets. Analytical Chemistry 78, 1401-4 (2006).

71. Barbulovic-Nad, I., Yang, H., Park, P.S. \& Wheeler, A.R. Digital microfluidics for cellbased assays. Lab on a Chip 8, 519-26 (2008).

72. Miller, E.M. \& Wheeler, A.R. A Digital Microfluidic Approach to Homogeneous Enzyme Assays. Analytical Chemistry 80, 1614-1619 (2008).

73. Taniguchi, T., Torii, T. \& Higuchi, T. Chemical reactions in microdroplets by electrostatic manipulation of droplets in liquid media. Lab on a Chip 2, 19-23 (2002).

74. Srinivasan, V. Droplet-based microfluidic lab-on-a-chip for glucose detection. Analytica Chimica Acta 507, 145-150 (2004). 
75. Srinivasan, V., Pamula, V.K. \& Fair, R.B. An integrated digital microfluidic lab-on-a-chip for clinical diagnostics on human physiological fluids. Lab on a Chip 4, 310-5 (2004).

76. Nichols, K.P. \& Gardeniers, H.J.G.E. A digital microfluidic system for the investigation of pre-steady-state enzyme kinetics using rapid quenching with MALDI-TOF mass spectrometry. Analytical Chemistry 79, 8699-704 (2007).

77. Chang, Y.-H., Lee, G.-B., Huang, F.-C., Chen, Y.-Y. \& Lin, J.-L. Integrated polymerase chain reaction chips utilizing digital microfluidics. Biomedical Microdevices 8, 215-25 (2006).

78. Malmsten, M. Formation of Adsorbed Protein Layers. Journal of Colloid and Interface Science 207, 186-199 (1998).

79. Giacomelli, C.E. \& Norde, W. Influence of hydrophobic Teflon particles on the structure of amyloid beta-peptide. Biomacromolecules 4, 1719-26 (2003).

80. Bayiati, P. et al. Electrowetting on plasma-deposited fluorocarbon hydrophobic films for biofluid transport in microfluidics. Journal of Applied Physics 101, 103306 (2007).

81. Yoon, J.-yeol \& Garrell, R.L. Preventing Biomolecular Adsorption in ElectrowettingBased Biofluidic Chips. Analytical Chemistry 75, 5097-5102 (2003).

82. Krupenkin, T.N., Taylor, J.A., Schneider, T.M. \& Yang, S. From rolling ball to complete wetting: the dynamic tuning of liquids on nanostructured surfaces. Langmuir 20, 3824-7 (2004).

83. Boduroglu, S., Cetinkaya, M., Dressick, W.J., Singh, A. \& Demirel, M.C. Controlling the wettability and adhesion of nanostructured poly-(p-xylylene) films. Langmuir 23, 11391-5 (2007).

84. Herbertson, D.L., Evans, C.R., Shirtcliffe, N.J., McHale, G. \& Newton, M.I. Electrowetting on superhydrophobic SU-8 patterned surfaces. Sensors and Actuators A: Physical 130-131, 189-193 (2006). 
85. Merrill, E.W. Poly(ethylene oxide) star molecules: synthesis, characterization, and applications in medicine and biology. Journal of Biomaterials Science, Polymer Edition 5 , $1-11$ (1993).

86. Jeon, S.I., Lee, J.H., Andrade, J.D. \& De Gennes, P.G. Protein-Surface Interactions in the Presence of Polyethylene Oxide 1. Simplified theory. Journal of Colloid and Interface Science 142, 145-158 (1991).

87. Marsh, L.H. et al. Adsorbed poly(ethyleneoxide)-poly(propyleneoxide) copolymers on synthetic surfaces: spectroscopy and microscopy of polymer structures and effects on adhesion of skin-borne bacteria. Journal of Biomedical Materials Research 61, 641-52 (2002).

88. McClain, M.A. et al. Microfluidic Devices for the High-Throughput Chemical Analysis of Cells. Analytical Chemistry 75, 5646-5655 (2003).

89. Houchin-Ray, T., Whittlesey, K.J. \& Shea, L.D. Spatially patterned gene delivery for localized neuron survival and neurite extension. Molecular Therapy 15, 705-12 (2007).

90. Sodhi, R.N.S. Time-of-flight secondary ion mass spectrometry (TOF-SIMS):--versatility in chemical and imaging surface analysis. The Analyst 129, 483-7 (2004).

91. Fauth, J.-M., Schweiger, A., Braunschweiler, L., Forrer, J. \& Ernst, R.R. Elimination of Unwanted Echoes and Reduction of Dead Time in Three-Pulse Electron Spin-Echo Spectroscopy. Journal of Magnetic Resonance 66, 74-85 (1986).

92. Quinn, A., Sedev, R. \& Ralston, J. Contact angle saturation in electrowetting. The Journal of Physical Chemistry B 109, 6268-75 (2005).

93. Cho, S.K., Moon, H. \& Kim, C.-jin Creating, Transporting, Cutting, and Merging Liquid Droplets by Electrowetting-Based Actuation for Digital Microfluidic Circuits. Journal of Microelectromechanical Systems 12, 70-80 (2003).

94. Amiji, M. \& Park, K. Prevention of protein adsorption and platelet adhesion on surfaces by PEO/PPO/PEO triblock copolymers. Biomaterials 13, 682-92 (1992). 
95. Nakashima, K., Anzai, T. \& Fujimoto, Y. Fluorescence Studies on the Properties of a Pluronic F68 Micelle. Langmuir 10, 658-661 (1994).

96. Desai, P.R., Jain, N.J., Sharma, R.K. \& Bahadur, P. Effect of additives on the micellization of PEO/PPO/PEO block copolymer F127 in aqueous solution. Colloids and Surfaces A: Physicochemical and Engineering Aspects 178, 57-69 (2001).

97. Malmsten, M. \& Lindman, B. Self-Assembly in Aqueous Block Copolymer Solutions. Macromolecules 25, 5440-5445 (1992).

98. Prud'homme, R.K., Wu, G. \& Schneider, D.K. Structure and Rheology Studies of Poly (oxyethylene-oxypropylene-oxyethylene) Aqueous Solution. Langmuir 12, 4651-4659 (1996).

99. Wanka, G., Hoffmann, H. \& Ulbricht, W. Phase Diagrams and Aggregation Behavior of Poly(oxyethylene)-Poly(oxypropylene)-Poly(oxyethylene) Triblock Copolymers in Aqueous Solutions. Macromolecules 27, 4145-4159 (1994).

100. Significance reported here are all $(\mathrm{p}<0.05)$. For FITC-casein, spots formed from solutions containing $0.4 \%$ Pluronic F127 exhibited less fluorescence than $0.08 \%$, and exhibited equivalent fluorescence to $0.8 \%$. For FITC-BSA, all conditions were equivalent. For AlexaFluor 488-Fb, $0.08 \%$ exhibited less fluorescence than $0.8 \%$, and exhibited equivalent fluorescence to $0.4 \%$. Because no trend was observed and because the differences were small, we chose to focus on the lowest concentration, $0.08 \% \mathrm{~F} 127$.

101. Nojiri, C. et al. Blood compatibility of PEO grafted polyurethane and HEMA/styrene block copolymer surfaces. Journal of Biomedical Materials Research 24, 1151-71 (1990).

102. Wazawa, T., Ishizuka-Katsura, Y., Nishikawa, S., Iwane, A.H. \& Aoyama, S. Grafting of poly(ethylene glycol) onto poly(acrylic acid)-coated glass for a protein-resistant surface. Analytical Chemistry 78, 2549-56 (2006).

103. Stilbs, P. Fourier Transform Pulse-Gradient Spin-Echo Studies of Molecular Diffusion. Progress in Nuclear Magnetic Resonance Spectroscopy 19, 1-45 (1987). 
104. Karger, J., Pfeifer, H. \& Heink, W. Principles and Applications of Self-Diffusion Measurements by Nuclear Magnetic Resonance. Advances in Magnetic and Optical Resonance 12, 1-89 (1988).

105. Price, W.S. Pulse-Field Gradient Nuclear Magnetic Resonance as a Tool for Studying Translational Diffusion: Part 1. Basic Theory. Concepts in Magenetic Resonance 9, 299336 (1997).

106. Price, W.S. Pulse-Field Gradient Nuclear Magnetic Resonance as a Tool for Studying Translational Diffussion Part 2. Experimental Aspects. Concepts in Magenetic Resonance 10, 197-237 (1998).

107. Kozer, N., Kuttner, Y.Y., Haran, G. \& Schreiber, G. Protein-protein association in polymer solutions: from dilute to semidilute to concentrated. Biophysical Journal 92, 2139-49 (2007).

108. Bhat, R. \& Timasheff, S.N. Steric exclusion is the principal source of the preferential hydration of proteins in the presence of polyethylene glycols. Protein science: $a$ publication of the Protein Society 1, 1133-43 (1992).

109. Timasheff, S.N. Protein hydration, thermodynamic binding, and preferential hydration. Biochemistry 41, 13473-82 (2002).

110. Matthew, J.E., Nazario, Y.L., Roberts, S.C. \& Bhatia, S.R. Effect of mammalian cell culture medium on the gelation properties of Pluronic F127. Biomaterials 23, 4615-9 (2002).

111. Kamil, S.H. et al. Tissue engineering of a human sized and shaped auricle using a mold. The Laryngoscope 114, 867-70 (2004).

112. Hyclone Media: Cho Cell Culture Platform Media. at <http://www.hyclone.com/media/cho.htm>

113. Chen, C., Even, M.A. \& Chen, Z. Detecting Molecular-Level Chemical Structure and Group Orientation of Amphiphilic PEO-PPO-PEO Copolymers at Solution / Air and Solid 
/ Solution Interfaces by SFG Vibrational Spectroscopy. Macromolecules 36, 4478-4484 (2003).

114. Phipps, J.S., Richardson, R.M., Cosgrove, T. \& Eaglesham, a. Neutron reflection studies of copolymers at the hexane/water interface. Langmuir 9, 3530-3537 (1993).

115. Rai, A.J. et al. HUPO Plasma Proteome Project specimen collection and handling: towards the standardization of parameters for plasma proteome samples. Proteomics 5, 3262-77 (2005).

116. Zhang, X., Li, L., Wei, D., Yap, Y. \& Chen, F. Moving cancer diagnostics from bench to bedside. Trends in Biotechnology 25, 166-73 (2007).

117. Freire, S.L.S. \& Wheeler, A.R. Proteome-on-a-chip: mirage, or on the horizon? Lab on a chip 6, 1415-23 (2006).

118. Jiang, Y. \& Lee, C.S. On-line coupling of micro-enzyme reactor with micro-membrane chromatography for protein digestion, peptide separation, and protein identification using electrospray ionization mass spectrometry. Journal of Chromatography A 924, 315-22 (2001).

119. Peterson, D.S., Rohr, T., Svec, F. \& Fréchet, J.M.J. Enzymatic microreactor-on-a-chip: protein mapping using trypsin immobilized on porous polymer monoliths molded in channels of microfluidic devices. Analytical Chemistry 74, 4081-8 (2002).

120. Crabtree, H.J., Cheong, E.C.S., Tilroe, D.A. \& Backhouse, C.J. Microchip Injection and Separation Anomalies Due to Pressure Effects. Analytical Chemistry 73, 4079-4086 (2001).

121. Walker, G.M. \& Beebe, D.J. A passive pumping method for microfluidic devices. Lab on a Chip 2, 131-4 (2002).

122. Wheeler, A.R. Chemistry. Putting electrowetting to work. Science 322, 539-40 (2008).

123. Abdelgawad, M. \& Wheeler, A.R. The Digital Revolution: A New Paradigm for Microfluidics. Advanced Materials 21, 920-925 (2009). 
124. Sista, R. et al. Development of a digital microfluidic platform for point of care testing. Lab on a Chip 8, 2091-104 (2008).

125. Sista, R.S. et al. Heterogeneous immunoassays using magnetic beads on a digital microfluidic platform. Lab on a Chip 8, 2188-96 (2008).

126. Chatterjee, D., Ytterberg, A.J., Son, S.U., Loo, J.A. \& Garrell, R.L. Integration of Protein Processing Steps on a Digital Microfluidics Platform for Analysis by MALDI-MS. Micro. Tot. Anal. Sys.; Chemical and Biological Microsystems Society 1072-1074 (2008).

127. Chatterjee, D. Ph. D. Dissertation, University of California, Los Angeles. (2008).

128. Luk, V.N., Mo, G.C. \& Wheeler, A.R. Pluronic additives: a solution to sticky problems in digital microfluidics. Langmuir 24, 6382-6389 (2008).

129. Getz, E.B., Xiao, M., Chakrabarty, T., Cooke, R. \& Selvin, P.R. A comparison between the sulfhydryl reductants tris(2-carboxyethyl)phosphine and dithiothreitol for use in protein biochemistry. Analytical Biochemistry 273, 73-80 (1999).

130. Ryle, A.P., Sanger, F., Smith, L.F. \& Kitai, R. The disulphide bonds of insulin. The Biochemical Journal 60, 541-56 (1955).

131. Kirley, T.L. Reduction and fluorescent labeling of cyst(e)ine-containing proteins for subsequent structural analyses. Analytical Biochemistry 180, 231-6 (1989).

132. Uchiyama, S., Santa, T., Okiyama, N., Fukushima, T. \& Imai, K. Fluorogenic and fluorescent labeling reagents with a benzofurazan skeleton. Biomedical Chromatography 15, 295-318 (2001).

133. Jones, L.J., Upson, R.H., Haugland, R.P., Panchuk-Voloshina, N. \& Zhou, M. Quenched BODIPY dye-labeled casein substrates for the assay of protease activity by direct fluorescence measurement. Analytical Biochemistry 251, 144-52 (1997).

134. Ren, H., Fair, R.B. \& Pollack, M.G. Automated on-chip droplet dispensing with volume control by electro-wetting actuation and capacitance metering. Sensors and Actuators B: Chemical 98, 319-327 (2004). 
135. Gong, J. \& Kim, C.-J. All-electronic droplet generation on-chip with real-time feedback control for EWOD digital microfluidics. Lab on a Chip 8, 898-906 (2008).

136. Hunyadi-Gulyás, É. \& Medzihradszky, K.F. Factors that contribute to the complexity of protein digests. Drug Discovery Today: Targets 3, S3-S10 (2004).

137. Abdelgawad, M., Freire, S.L.S., Yang, H. \& Wheeler, A.R. All-terrain droplet actuation. Lab on a Chip 8, 672-7 (2008).

138. Abdelgawad, M., Watson, M.W.L. \& Wheeler, A.R. Hybrid microfluidics: a digital-tochannel interface for in-line sample processing and chemical separations. Lab on a Chip $\mathbf{9}$, 1046-51 (2009).

139. Hanash, S. Disease proteomics. Nature 422, 226-32 (2003).

140. Ludwig, J. a \& Weinstein, J.N. Biomarkers in cancer staging, prognosis and treatment selection. Nature reviews. Cancer 5, 845-56 (2005).

141. Swami, M. Proteomics: A discovery strategy for novel cancer biomarkers. Nature Reviews. Cancer 10, 597-597 (2010).

142. Anderston, N.L., Matheson, A.D. \& Steine, S. Proteomics: applications in basic and applied biology. Current Opinion in Biotechnology 11, 2099-2104 (2000).

143. Steiner, S. Proteomics: Applications and opportunities in preclinical drug development Proteomics and 2-DE. Proteome (2000).

144. Thome-Kromer, B. et al. Toward the identification of liver toxicity markers: a proteome study in human cell culture and rats. Proteomics 3, 1835-62 (2003).

145. Zhou, J., Cai, Z.-H., Li, L., Gao, Y.-F. \& Hutchinson, T.H. A proteomics based approach to assessing the toxicity of bisphenol A and diallyl phthalate to the abalone (Haliotis diversicolor supertexta). Chemosphere 79, 595-604 (2010).

146. Lee, J.-M. \& Kohn, E.C. Proteomics as a guiding tool for more effective personalized therapy. Annals of Oncology 21 Suppl 7, vii205-vii210 (2010). 
147. López-Ferrer, D. et al. Sample treatment for protein identification by mass spectrometrybased techniques. Trends in Analytical Chemistry 25, 996-1005 (2006).

148. Schaefer, H. et al. Tryptic transpeptidation products observed in proteome analysis by liquid chromatography-tandem mass spectrometry. Proteomics 5, 846-52 (2005).

149. Lundell, N. \& Schreitmüller, T. Sample preparation for peptide mapping--A pharmaceutical quality-control perspective. Analytical Biochemistry 266, 31-47 (1999).

150. Monzo, A., Sperling, E. \& Guttman, A. Proteolytic enzyme-immobilization techniques for MS-based protein analysis. Trends in Analytical Chemistry 28, 854-864 (2009).

151. Krenková, J. \& Foret, F. Immobilized microfluidic enzymatic reactors. Electrophoresis 25, 3550-63 (2004).

152. Lion, N. et al. Microfluidic systems in proteomics. Electrophoresis 24, 3533-62 (2003).

153. Sigma-Aldrich http://www.sigmaaldrich.com/life-science/proteomics/massspectrometry/trypsin-spin-column.html. (2010).

154. Bao, H., Lui, T., Zhang, L. \& Chen, G. Infrared-assisted proteolysis using trypsinimmobilized silica microspheres for peptide mapping. Proteomics 9, 1114-7 (2009).

155. Poroszyme® Immobilized Trypsin Cartridge http://www.appliedbiosystems.com. (2010).

156. Stigter, E.C. a, de Jong, G.J. \& van Bennekom, W.P. Development of an open-tubular trypsin reactor for on-line digestion of proteins. Analytical and Bioanalytical Chemistry 389, 1967-77 (2007).

157. Cooper, J.W., Chen, J., Li, Y. \& Lee, C.S. Membrane-Based Nanoscale Proteolytic Reactor Enabling Protein Digestion, Peptide Separation, Spectrometry. Analytical Chemistry 75, 1067-1074 (2003).

158. Mateo, C. et al. Glyoxyl agarose: A fully inert and hydrophilic support for immobilization and high stabilization of proteins. Enzyme and Microbial Technology 39, 274-280 (2006). 
159. Zubtsov, D. a et al. Comparison of surface and hydrogel-based protein microchips. Analytical Biochemistry 368, 205-13 (2007).

160. Choi, D., Lee, W., Park, J. \& Koh, W. Preparation of poly(ethylene glycol) hydrogels with different network structures for the application of enzyme immobilization. Bio-medical Materials and Engineering 18, 345-356 (2008).

161. De Hoog, H.-P.M., Arends, I.W.C.E., Rowan, A.E., Cornelissen, J.J.L.M. \& Nolte, R.J.M. A hydrogel-based enzyme-loaded polymersome reactor. Nanoscale 2, 709-16 (2010).

162. Fiddes, L.F. et al. Hydrogel Discs for Digital Microfluidics. Biomicrofluidics DOI: 10.10, (2012).

163. Jebrail, M.J. et al. Digital Microfluidics for Automated Proteomic Processing. Journal of Visualized Experiments 33, (2009).

164. Miller, E.M., Ng, A.H.C., Uddayasankar, U. \& Wheeler, A.R. A digital microfluidic approach to heterogeneous immunoassays. Analytical and Bioanalytical Chemistry 399, 337-45 (2011).

165. Blanco, R.M., Calvete, J.J. \& Guisán, J.M. Immobilizaztion-stabilization of Enzymes; Variables that Control the Intensity of the Trypsin (Amine)-agarose (Aldehyde) Multipoint Attachment. Enzyme Microbiology Technology 11, 353-359 (1989).

166. Guisan, J.M., Bastida, A., Cuesta, C. \& Rosell, C.M. Immobilization-Stabilization of alpha-Chymotrypsin by Covalent Attachment to Aldehyde-Agarose Gels. Biotechnology and Bioengineering 38, 1144-1152 (1991).

167. Guisan, J.M., Bastida, A., Blanco, R.M., Fernandes-Lafuente, R. \& Garcia-Junceda, E. Immobilization of Enzymes on Glyoxyl Agarose: Strategies for Enzyme Stabilization by Multipoint Attachment. Immobilization of Enzymes and Cells 277-287 (1997).

168. Gatlin, C.L., Eng, J.K., Cross, S.T., Detter, J.C. \& Yates 3rd, J.R. Automated Identification of Amino Acid Sequence Variations in Proteins by HPLC/Microspray Tandem Mass Spectrometry. Analytical Chemistry 72, 757-763 (2000). 
169. Choudhary, G., Wu, S.-L., Shieh, P. \& Hancock, W.S. Multiple enzymatic digestion for enhanced sequence coverage of proteins in complex proteomic mixtures using capillary LC with ion trap MS/MS. Journal of Proteome Research 2, 59-67

170. Omenn, G.S. Advancement of biomarker discovery and validation through the HUPO plasma proteome project. Disease Markers 20, 131-4 (2004).

171. Yamaguchi, H. et al. Rapid and efficient proteolysis for proteomic analysis by proteaseimmobilized microreactor. Electrophoresis 30, 3257-64 (2009).

172. Fan, H., Bao, H., Zhang, L. \& Chen, G. Immobilization of trypsin on poly(ureaformaldehyde)-coated fiberglass cores in microchip for highly efficient proteolysis. Proteomics 11, 3420-3 (2011).

173. Lee, J., Musyimi, H.K., Soper, S. a \& Murray, K.K. Development of an automated digestion and droplet deposition microfluidic chip for MALDI-TOF MS. Journal of the American Society for Mass Spectrometry 19, $964-72$ (2008).

174. Astorga-Wells, J., Bergman, T. \& Jörnvall, H. Multistep microreactions with proteins using electrocapture technology. Analytical Chemistry 76, 2425-9 (2004).

175. Sivaraman, T., Kumar, T.K., Jayaraman, G. \& Yu, C. The mechanism of 2,2,2trichloroacetic acid-induced protein precipitation. Journal of Protein Chemistry 16, 291-7 (1997).

176. Xu, Z., Xie, Q. \& Zhou, H.-M. Trichloroacetic acid-induced molten globule state of aminoacylase from pig kidney. Journal of Protein Chemistry 22, 669-75 (2003).

177. Speers, A.E. \& Wu, C.C. Proteomics of integral membrane proteins--theory and application. Chemical Reviews 107, 3687-714 (2007).

178. Kim, S.C. et al. A clean, more efficient method for in-solution digestion of protein mixtures without detergent or urea. Journal of Proteome Research 5, 3446-52 (2006). 
179. Liu, H., Sadygov, R.G. \& Yates, J.R. A Model for Random Sampling and Estimation of Relative Protein Abundance in Shotgun Proteomics. Analytical Chemistry 76, 4193-4201 (2004).

180. Shih, S.C.C., Fobel, R., Kumar, P. \& Wheeler, A.R. A feedback control system for highfidelity digital microfluidics. Lab on a Chip 11, 535-40 (2011).

181. Yang, H., Mudrik, J.M., Jebrail, M.J. \& Wheeler, A.R. A digital microfluidic method for in situ formation of porous polymer monoliths with application to solid-phase extraction. Analytical Chemistry 83, 3824-30 (2011).

182. Osada, Y., Ping Gong, J. \& Tanaka, Y. Polymer Gels. Journal of Macromolecular Science, Part C: Polymer Reviews 44, 87-112 (2004).

183. Tumarkin, E. \& Kumacheva, E. Microfluidic generation of microgels from synthetic and natural polymers. Chemical Society Reviews 38, 2161-8 (2009).

184. Eddington, D. Flow control with hydrogels. Advanced Drug Delivery Reviews 56, 199210 (2004).

185. Lee, S., Eddington, D.T., Kim, Y., Kim, W. \& Beebe, D.J. Control Mechanism of an Organic Self-Regulating Microfluidic System. Journal of Microelectromechanical Systems 12, 848-854 (2003).

186. Rivest, C. et al. Microscale Hydrogels for Medicine and Biology: Synthesis, Characteristics and Applications. Journal of Mechanics of Materials and Structures $\mathbf{6}$, 1103-1119 (2007).

187. Huang, X. et al. Smart microgel catalyst with modulatory glutathione peroxidase activity. Soft Matter 5, 1905 (2009).

188. Retama, J.R., Lopez-Ruiz, B. \& Lopez-Cabarcos, E. Microstructural modifications induced by the entrapped glucose oxidase in cross-linked polyacrylamide microgels used as glucose sensors. Biomaterials 24, 2965-2973 (2003). 
189. Courtenay, V.D., Selby, P.J., Smith, I.E., Mills, J. \& Peckham, M.J. Growth of human tumour cell colonies from biopsies using two soft-agar techniques. British Journal of Cancer 38, 77-81 (1978).

190. Flickinger, S.T. et al. Quorum sensing between Pseudomonas aeruginosa biofilms accelerates cell growth. Journal of the American Chemical Society 133, 5966-75 (2011).

191. Plouffe, B.D., Brown, M.A., Iyer, R.K., Radisic, M. \& Murthy, S.K. Controlled Capture and Release of Cardiac Fibroblasts using Peptide-Functionalized Alginate Gels in Microfluidic Channels. Lab on a Chip 9, 1507-1510 (2009).

192. Hatch, A., Hansmann, G. \& Murthy, S.K. Engineered alginate hydrogels for effective microfluidic capture and release of endothelial progenitor cells from whole blood. Langmuir 27, 4257-64 (2011).

193. McGuigan, A.P. et al. Cell encapsulation in sub-mm sized gel modules using replica molding. PloS one 3, e2258 (2008).

194. Eun, Y.-jin, Utada, A.S., Copeland, M.F., Takeuchi, S. \& Weibel, D.B. Encapsulating Bacteria in Agarose Microparticles using Microfluidics for High-Throughput Cell Analysis and Isolation. American Chemical Society Chemical Biology 6, 260-266 (2011).

195. Park, T.G. \& Hoffman, A.S. Synthesis and characterization of $\mathrm{pH}-$ and/or temperaturesensitive hydrogels. Jounral of Applied Polymer 46, 659-671 (1992).

196. Hovgaard, L. \& Brondsted, H. Dextran hydrogels for colon-specific drug delivery. Journal of Controlled Release 36, 159-166 (1995).

197. Boissière, M. et al. Turning biopolymer particles into hybrid capsules: the example of silica/alginate nanocomposites. Journal of Materials Chemistry 16, 1178-1182 (2006).

198. Islam, M.T., Ciotti, S. \& Ackermann, C. Rheological Characterization of Topical Carbomer Gels Neutralized to Different pH. Pharmaceutical Research 21, 1192-1199 (2004). 
199. Lopez, V.C., Hadgraft, J. \& Snowden, M.J. The use of colloidal microgels as a (trans)dermal drug delivery system. International Journal of Pharmaceutics 292, 137-47 (2005).

200. Popanda, O. et al. Radiation-induced DNA damage and repair in lymphocytes from breast cancer patients and their correlation with acute skin reactions to radiotherapy. International Journal of Radiation Oncology Biology Physics 55, 1216-1225 (2003).

201. de Vicente, J., Stokes, J.R. \& Spikes, H. a. Soft lubrication of model hydrocolloids. Food Hydrocolloids 20, 483-491 (2006).

202. Khan, A.A., Khan, H.M. \& Delincée, H. DNA Comet Assay - a rapid screening method for detection of irradiated cereals and tree nuts. Food Control 16, 141-146 (2005).

203. Villarini, M., Scassellati-Sforzolini, G., Moretti, M. \& Pasquini, R. In vitro genotoxicity of terbutryn evaluated by the alkaline single-cell microgel-electrophoresis "comet" assay. Cell Biology and Toxicology 16, 285-92 (2000).

204. Koh, W.-G. \& Pishko, M. Immobilization of multi-enzyme microreactors inside microfluidic devices. Sensors \& Actuators B 106, 335-342 (2005).

205. Koh, W.-G. \& Pishko, M.V. Fabrication of cell-containing hydrogel microstructures inside microfluidic devices that can be used as cell-based biosensors. Analytical and Bioanalytical Chemistry 385, 1389-1397 (2006).

206. Srinivasan, A., Bach, H., Sherman, D.H. \& Dordick, J.S. Bacterial P450-catalyzed polyketide hydroxylation on a microfluidic platform. Biotechnology and Bioengineering 88, 528-35 (2004).

207. Wong, A.P., Perez-Castillejos, R., Love, J.C. \& Whitesides, G.M. Partitioning microfluidic channels with hydrogel to construct tunable 3-D cellular microenvironments. Biomaterials 29, 1853-61 (2008).

208. Ghajar, C.M. et al. The effect of matrix density on the regulation of 3-D capillary morphogenesis. Biophysical Journal 94, 1930-41 (2008). 
209. Liu, Y., Liu, B., Yang, P. \& Girault., H.H. Microfluidic enzymatic reactors for proteome research. Analytical and Bioanalytical Chemistry 390, 227-229 (2008).

210. Sundararaghavan, H.G., Monteiro, G. a, Firestein, B.L. \& Shreiber, D.I. Neurite growth in 3D collagen gels with gradients of mechanical properties. Biotechnology and Bioengineering 102, 632-43 (2009).

211. Kuckling, D., Harmon, M.E. \& Frank, C.W. Photo-Cross-Linkable PNIPAAm Copolymers. 1. Synthesis and Characterization of Constrained Temperature-Responsive Hydrogel Layers. Macromolecules 35, 6377-6383 (2002).

212. Sundararaghavan, H.G., Masand, S.N. \& Shreiber, D.I. Microfluidic generation of haptotactic gradients through 3D collagen gels for enhanced neurite growth. Journal of Neurotrauma 28, 2377-87 (2011).

213. Shah, G.J., Ohta, A.T., Chiou, E.P.-Y., Wu, M.C. \& Kim, C.-J. EWOD-driven droplet microfluidic device integrated with optoelectronic tweezers as an automated platform for cellular isolation and analysis. Lab on a Chip 9, 1732-9 (2009).

214. Fan, S.-K., Huang, P.-W., Wang, T.-T. \& Peng, Y.-H. Cross-scale electric manipulations of cells and droplets by frequency-modulated dielectrophoresis and electrowetting. Lab on a Chip 8, 1325-31 (2008).

215. Barbulovic-Nad, I., Au, S.H. \& Wheeler, A.R. A microfluidic platform for complete mammalian cell culture. Lab on a Chip 10, 1536-42 (2010).

216. Au, S.H., Shih, S.C. \& A.R. Wheeler Integrated microbioreactor for culture and analysis of bacteria, algae and yeast. Biomedical Microdevices 13, 41-50 (2011).

217. Witters, D. et al. Biofunctionalization of electrowetting-on-dielectric digital microfluidic chips for miniaturized cell-based applications. Lab on a Chip 11, 2790-4 (2011).

218. Vergauwe, N. et al. A versatile electrowetting-based digital microfluidic platform for quantitative homogeneous and heterogeneous bio-assays. Journal of Micromechanics and Microengineering 21, 054026 (2011). 
219. Srigunapalan, S., Eydelnant, I.A., Simmons, C.A. \& Wheeler, A.R. A digital microfluidic platform for primary cell culture and analysis. Lab on a Chip 12, 369-75 (2012).

220. Fair, R.B. et al. Chemical and Biological Applications of Digital-Microfluidic Devices. Design \& Test of Computers, IEEE 24, 10-24 (2007).

221. Jebrail, M.J. et al. Synchronized Synthesis of Peptide-Based Macrocycles by Digital Microfluidics. Angewandte Chemie International Edition 49, 8625-8629 (2010).

222. Rani, S.A., Pitts, B. \& Stewart, P.S. Rapid Diffusion of Fluorescent Tracers into Staphylococcus epidermidis Biofilms Visualized by Time Lapse Microscopy. Antimicrobial Agents and Chemotherapy 49, 728-732 (2005).

223. Pernodet, N., Maaloum, M. \& Tinland, B. Pore size of agarose gels by atomic force microscopy. Electrophoresis 18, 55-58 (1997).

224. Grinnell, F. Fibroblast biology in three-dimensional collagen matrices. Trends in Cell Biology 13, 264-269 (2003).

225. Griffith, L.G. \& Swartz, M.A. Capturing complex 3D tissue physiology in vitro. Nature Reviews Molecular Cell Biology 7, 211-224 (2006).

226. Folch, A. \& Toner, M. Microengineering of Cellular Interactions. Annual Review of Biomedical Engineering 2, 227-256 (2000).

227. Barralet, J.E. et al. Comparison of bone marrow cell growth on 2D and 3D alginate hydrogels. Journal of Materials Science: Materials in Medicine 16, 515-9 (2005).

228. Fournier, M.L., Gilmore, J.M., Martin-Brown, S.A. \& Washburn, M.P. Multidimensional separations-based shotgun proteomics. Chemical Reviews 107, 3654-3686 (2007). 